\title{
ORGANIC MOLECULAR FILMS ON METAL AND GRAPHENE SURFACES STUDIED WITH LEEM
}

Fawad Salman Khokhar 
Doctoral committee:

Chairman

Prof. dr. G. van der Steenhoven, University of Twente

Promotor

Prof. dr. ir. B. Poelsema, University of Twente

Assistant-promotors

Dr. R. van Gastel, University of Twente

Dr. G. Hlawacek, University of Twente

Members

Ao. Univ. Prof. dr. rer. nat. Ch. Teichert, Montanuniversitaet Leoben

Prof. dr. ir. H.J.W. Zandvliet, University of Twente

Prof. dr. ir. L. Lefferts, University of Twente

The work described in this thesis was performed at Physics of Interfaces and Nanomaterials at the Faculty of Science and Technology, University of Twente, Enschede, The Netherlands.

This work is part of the research programme of the Foundation for Fundamental Research on Matter (FOM) which is part of the Netherlands Organisation for Scientific Research (NWO), (project: 04PR2318).

Fawad Salman Khokhar

Organic Molecular Films on Metal and Graphene Surfaces studied with LEEM Ph.D. thesis, University of Twente, Enschede, The Netherlands.

ISBN: 978-90-365-3269-3

DOI-number: 10.3990/1.9789036532693

URL: http://dx.doi.org/10.3990/1.9789036532693

Cover: Fawad Salman Khokhar

Printed by: Ipskamp Drukkers, Enschede, The Netherlands. 


\title{
ORGANIC MOLECULAR FILMS ON METAL AND GRAPHENE SURFACES STUDIED WITH LEEM
}

\author{
DISSERTATION
}

to obtain

the degree of doctor at the University of Twente, on the authority of the rector magnificus, prof. dr. H. Brinksma, on account of the decision of the graduation committee, to be publicly defended on Wednesday the $11^{\text {th }}$ of January, 2012 at 16:45hrs

BY

Fawad Salman Khokhar

born on $28^{\text {th }}$ of January, 1979

in Gujranwala, Pakistan 
This dissertation has been approved by:

Prof.dr.ir. B. Poelsema (Promotor)

Dr. R. van Gastel (Assistant-promotor)

Dr. G. Hlawacek (Assistant-promotor) 


\section{Contents}

1 Introduction 1

2 Experimental method $\quad 7$

2.1 Low Energy Electron Microscopy (LEEM) $\ldots \ldots \ldots \ldots$

2.1 .1 Contrast mechanisms . . . . . . . . . . . . . . 8

2.2 Low Energy Electron microDiffraction $(\mu$ LEED) $\ldots \ldots \ldots \ldots \ldots$

2.3 PhotoEmission Electron Microscopy (PEEM) _ . . . . . . . . . 10

2.4 Instrumentation . . . . . . . . . . . . . . . . . . . 11

2.5 Sample preparation . . . . . . . . . . . . . . . 12

2.5.1 $\mathrm{Cu}(001)$ surface preparation $\ldots \ldots \ldots \ldots \ldots . \ldots . \ldots 12$

2.5.2 $\operatorname{Ir}(111)$ surface preparation . . . . . . . . . . . . . 13

2.5.3 Graphene preparation . . . . . . . . . . . . . . . 14

3 Domain patterns and two-dimensional self-assembly 17

3.1 Self-assembly and competing interactions . . . . . . . . . . 18

3.2 Organic molecular surface structure $\ldots \ldots \ldots \ldots \ldots \ldots . \ldots 21$

3.3 Growth of TMA structures . . . . . . . . . . . . . 22

3.3.1 Results and observations . . . . . . . . . . . . . . 23

3.3.2 Analysis and discussion . . . . . . . . . . . . . . . . 24

3.4 Organic thin films and radiation damage $\ldots \ldots \ldots \ldots . \ldots 27$

4 Growth, structure, and thermal stability of BDA-domains on $\mathrm{Cu}(001) \quad 33$

4.1 Introduction . . . . . . . . . . . . . . . . . . . . . . 34

4.2 Experimental . . . . . . . . . . . . . . . . . . . . . 34

4.3 Results . . . . . . . . . . . . . . . . . . . 36

4.3.1 Growth at room temperature . . . . . . . . . . 36

4.3.2 Continued growth of the BDA domains at $448 \mathrm{~K} \ldots \ldots$

4.3.3 Thermal stability of the BDA domains . . . . . . . . . . 42

4.3 .4 Ostwald ripening . . . . . . . . . . . . . . 42

4.3.5 Temporal evolution of BDA domains at $448 \mathrm{~K} \ldots \ldots$. . . . 44

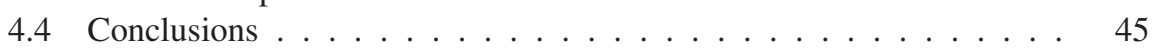

5 The role of topographical defects in growth of BDA film on graphene 49

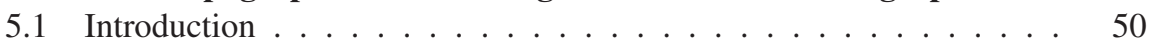

5.2 Experimental . . . . . . . . . . . . . . . 50 


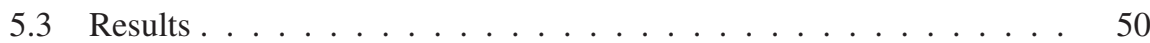

5.4 Discussion . . . . . . . . . . . . . . . . . 61

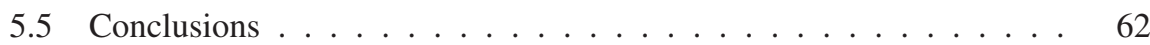

6 Smooth growth of 6P films on graphene for high efficiency electronics 65

6.1 Introduction . . . . . . . . . . . . . . . . . . . 66

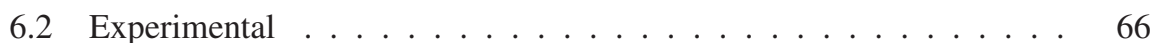

$6.36 \mathrm{P}$ growth . . . . . . . . . . . . . . . . . . . . . 68

6.4 Conclusions ........................ 71

7 Diffusion and submonolayer growth of $6 P$ on $\operatorname{Ir}(111)$ and graphene $\quad 75$

7.1 Introduction . . . . . . . . . . . . . . . . . 76

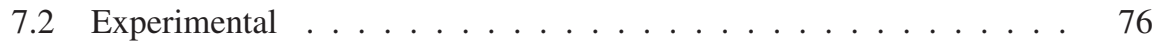

$7.36 \mathrm{P}$ on graphene flakes . . . . . . . . . . . . . . . . . . 76

$7.46 \mathrm{P}$ on $\operatorname{Ir}(111) \ldots \ldots \ldots \ldots$. . . . . . . . . . . . . . 80

7.5 Conclusions ........................ 83

8 The influence of substrate temperature on growth of $6 \mathrm{P}$ thin films on graphene

8.1 Introduction . . . . . . . . . . . . . . . . . . . 88

8.2 Experimental . . . . . . . . . . . . . . . . . . 88

8.3 Results and discussion . . . . . . . . . . . . . . . . . 89

8.3.1 Deposition of 6P at $320 \mathrm{~K} \ldots \ldots . \ldots . \ldots 89$

8.3.2 Deposition of $6 \mathrm{P}$ at $352 \mathrm{~K} \ldots \ldots . \ldots 95$

8.3.3 Deposition of $6 \mathrm{P}$ at $405 \mathrm{~K} \ldots \ldots$. . . . . . . . . . . . . . . . . . . . . . . . . . . . . 96

8.4 Conclusions . . . . . . . . . . . . . . . . . 98

$\begin{array}{ll}\text { Summary } & 110\end{array}$

$\begin{array}{lr}\text { Acknowledgements } & 111\end{array}$

$\begin{array}{lr}\text { Curriculum Vitae } & 113\end{array}$ 


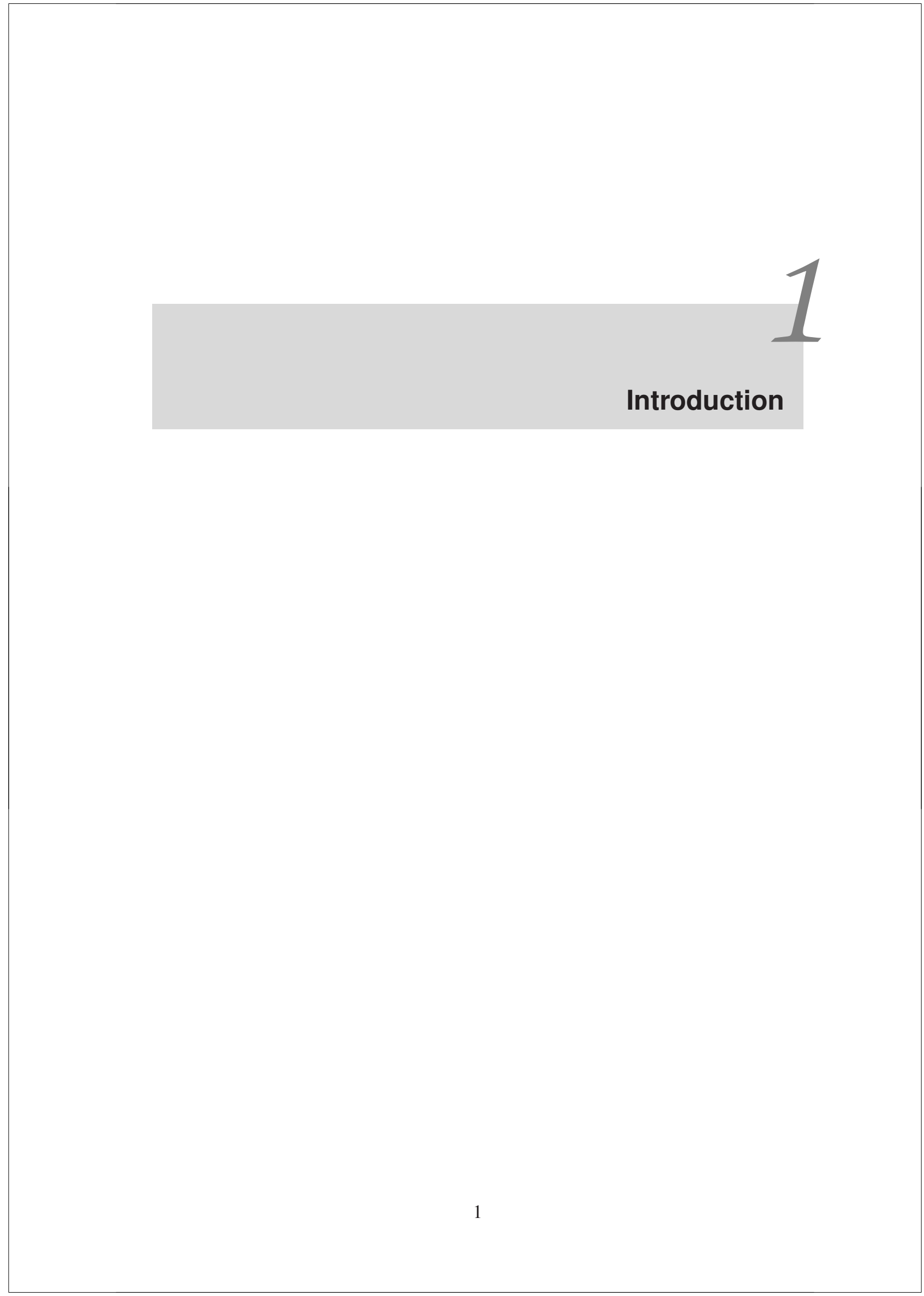


One of the grand goals of materials science is to be able to design, build, and understand functional materials with a precision that is equal to the size of the smallest possible entity, i.e. the size of an atom. This atomic-scale engineering of materials is a difficult, if not impossible, feat to achieve in three dimensions [1]. In two dimensions, it is already challenging enough. The large-scale, controlled positioning, application, and patterning of individual atoms and molecules on a substrate remains an elusive goal to this day [2]. Several techniques exist, but each has its drawbacks with respect to homogeneity of the fabricated structures, the defect density, or other relevant properties.

In this work, we explore a novel approach to the functionalization of substrates. The noncovalent patterning and functionalization of substrates is investigated to establish its effectiveness for future applications. The aim of our work is to directly image the formation of the patterns, and to expose and quantify the relevant thermodynamic growth parameters [3]. Features that are relevant to the positioning of the self-assembling entities can also be identified through this approach. In the formation of the final patterns, we aim to exploit long-range interactions that are normally present in self-assembling systems. Normally these long-range interactions are of an elastic, magnetic or electrostatic nature. For noncovalent molecules, both electrostatic and elastic interactions are anticipated to play a role [4].

The use of long-range stabilizing interactions has been demonstrated in self-assembling systems before [5]. Ordered arrays of dots, stripes, and interesting variations of these are seen in systems as different as ferromagnetic thin films [6-11], Langmuir monolayers at the air-water interface [12-15], and adsorbed atoms on solid surfaces [16-20]. The common feature in these widely varying systems is a competition between the long-range repulsive (electrostatic, magnetostatic, or elastic) interactions and short-range attractive interactions that leads to stabilization of domains with characteristic feature dimensions. Although, thermodynamic properties of domain structures resulting from competing interactions has been the subject of many theoretical studies $[6,7,9,12,14,15,21-28]$, quantitative information on the forces that drive pattern formation is lacking because it is difficult to measure forces on the length scale of self-assembly directly. Here, we take on this challenge by using Low Energy Electron Microscopy (LEEM) to directly image the pattern formation and dynamics [29].

Single crystalline metal substrates are used as a carrier for conjugated molecules and the pattern formation is investigated by direct imaging. Because of the metallic nature of some of the substrates that were used, noncovalent bonding is anticipated to play a role, either through adatoms or direct bonding of the molecules to the substrates [30]. This noncovalent bonding may be rather strong which would not be beneficial to the mobility of the molecules, and with it, the time on which a system self-assembles, it may also disrupt the conjugate nature of the used molecules, thereby altering their electronic structure and any electronic functionality that is pursued. Another approach that we have, therefore, pursued in producing novel nanomaterials is the application of the much-publicized material, graphene, as a substrate. It has very desirable optical, electronic, and mechanical properties [31-35]. Polycyclic aromatic compounds assemble into stable, ordered structures on epitaxial graphene. The sheets are fabricated using one of three methods: reduction of graphene oxide, chemical vapor deposition (CVD) [36], and the heating of $\mathrm{SiC}$ [37]. CVD has so far shown the great- 
est promise to fabricate large-scale, well-defined single layer graphene sheets [38]. It is used in this work to provide a platform for molecular assembly. Aromatic moieties typically interact strongly with the graphene and form well-defined $\pi$-bonds [39]. In addition to the $\pi$-bonds, intermolecular forces and intramolecular forces will contribute to the self-assembly. The result is a complex system in which many variables intertwine to eventually form a self-assembled structure. Direct opportunities for tailoring these systems arise through the synthesis of molecules with different backbone lengths and end groups. We have investigated two distinct types of molecules, 4,4'benzenedicarboxylic acid (BDA) and para-sexiphenyl (6P). The latter molecule has a much more explicit linear structure involving six benzene rings instead of two, and this change is reflected in both the growth behaviour of the molecule as well as in the final structures that are eventually observed.

An enormous drawback in our approach is the relative sensitivity of the molecules to external fields or probing particles [40]. The interaction that a Scanning Probe Microscope tip can locally have on organic matter is well-established [41-44]. On the other hand, charged particle beam systems tend to be equally damaging due to the high energy of the probing particle. Here, we investigate the applicability of LEEM to these delicate molecular layers. Aside from the real-space imaging capability of the instrument, it also provides valuable information on the molecular structure through Low Energy Electron Diffraction (LEED) and provides access to other thermodynamically relevant parameters through voltage-current characteristics that can be recorded both from real space and reciprocal space images [29].

This thesis is organized as follows. In Chapter 2, we detail the techniques that we have used and how they have been applied to the sensitive molecular systems. Both the aspect of real-time real space imaging will be discussed as well as the possibilities that a LEEM instrument has to structurally characterize the molecular films. We also highlight the sample preparation technique which is not an easy undertaking given that most metallic surfaces are easily contaminated by carbon residues from the experiments. In Chapter 3, we discuss the physical background that underpins the type of self-assembly that we investigate in more detail. A brief, but unsuccesful experiment using trimesic acid (TMA) molecules on the $\mathrm{Cu}(001)$ surface is presented. Chapter 4 details the experiments that followed the initial TMA measurements. A bigger molecule, BDA, was used to successfully form self-assembled domains on the $\mathrm{Cu}(001)$ surface. A further refinement of the balance between the molecule-substrate interactions on the one hand, and the molecule-molecule interactions on the other hand, is investigated in Chapter 5 where the same molecule was used to pattern graphene flakes on an $\operatorname{Ir}(111)$ substrate.

Having exposed the role of the substrate, the same experiments are repeated with the $6 \mathrm{P}$ molecule. Its structure and dynamics are investigated on graphene. Chapter 6 details the crystal structure that is formed in few layers high domains of 6P. Chapter 7 details the growth dynamics of the $6 \mathrm{P}$ on graphene. The temperature dependent structure that is observed is the subject of Chapter 8 and concludes our study of the noncovalent functionalization of substrates in two dimensions. 


\section{Bibliography}

[1] G.M. Whitesides and B. Grzybowski, Science 295 (2002), 2418.

[2] IBM Research (2011).

<http://www.zurich.ibm.com/st/storage/concept.html>

[3] R.M. Tromp and J.B. Hannon, Surf. Rev. Lett. 9 (2002), 1565.

[4] F.-J. Meyer zu Heringdorf, M.C. Reuter, and R.M. Tromp, Nature 412, (2001) 517.

[5] R. van Gastel, R. Plass, N.C. Bartelt, and G.L. Kellogg, Phys. Rev. Lett. 91 (2003), 055503.

[6] M. Seul and D. Andelman, Science 267 (1995), 476.

[7] T. Garel and S. Doniach, Phys. Rev. B 26 (1982), 325.

[8] M. Seul and R. Wolfe, Phys. Rev. A 46 (1992), 7519.

[9] S.A. Langer, R.E. Goldstein, and D.P. Jackson, Phys. Rev. A 46 (1992), 4894.

[10] A.B. Kashuba and V.L. Pokrovsky, Phys. Rev. B 48 (1993), 10335.

[11] A.J. Dickstein, S. Erramilli, R.E. Goldstein, D.P. Jackson, and S.A. Langer, Science 261 (1993), 1012.

[12] D.J. Keller, H.M. McConnell, and V.T. Moy, J. Phys. Chem. 90 (1986), 2311.

[13] D. Andelman, F. Brochard, and J.-F. Joanny, J. Chem. Phys. 86 (1987), 3673.

[14] H.M. McConnell, Annu. Rev. Phys. Chem. 42 (1991), 171.

[15] S.L. Keller and H.M. McConnell, Phys. Rev. Lett. 82 (1999), 1602.

[16] K. Kern, H. Niehus, A. Schatz, P. Zeppenfeld, J. George, and G. Comsa, Phys. Rev. Lett. 67 (1991), 855.

[17] P. Zeppenfeld, M.A. Krzyzowski, Ch. Romainczyk, R. David, G. Comsa, H. Röder, K. Bromann, H. Brune, and K. Kern, Surf. Sci. 342 (1995), L1131.

[18] K. Pohl, M. Bartelt, J. Figuera, N. Bartelt, J. Hrbek, and R. Hwang, Nature 397 (1999), 238.

[19] H. Ellmer, V. Repain, S. Rousset, B. Croset, M. Sotto, and P. Zeppenfeld, Surf. Sci. 476 (2001), 95.

[20] G.E. Thayer, V. Ozolins, A.K. Schmid, N.C. Bartelt, M. Asta, J.J. Hoyt, S. Chiang, and R.Q. Hwang, Phys. Rev. Lett. 86 (2001), 660.

[21] V.I. Marchenko, JETP Lett. 33 (1981), 381. 
[22] O.L. Alerhand, D. Vanderbilt, R.D. Meade, and J.D. Joannopoulos, Phys. Rev. Lett. 61 (1988), 1973.

[23] M.M. Hurley and S.J. Singer, Phys. Rev. B 46 (1992), 5783.

[24] D. Vanderbilt, Surf. Sci. Lett. 268 (1992), L300.

[25] C. Sagui and R.C. Desai, Phys. Rev. E 49 (1994), 2225.

[26] A.D. Stoycheva and S.J. Singer, Phys. Rev. E 65 (2002), 036706.

[27] K.-O. Ng and D. Vanderbilt, Phys. Rev. B 52 (1995), 2177.

[28] C. Sagui and R.C. Desai, Phys. Rev. E 52 (1995), 2807.

[29] E. Bauer, Surf. Rev. Lett. 5 (1998), 1275.

[30] J.V. Barth, Annu. Rev. Phys. Chem. 58, (2007) 375.

[31] F. Wang, Y. Zhang, C. Tian, C. Girit, A. Zettl, M. Crommie, and Y.R. Shen, Science, 320 (2008), 206.

[32] A.H. Castro Neto, F. Guinea, N.M.R. Peres, K.S. Novoselov, and A.K. Geim, Rev. Mod. Phys. 81 (2009), 109.

[33] M.D. Stoller, S. Park, Y. Zhu, J. An, and R.S. Ruoff, Nano Lett. 8, (2008), 3498.

[34] Yu-Ming Lin, K.A. Jenkins, A. Valdes-Garcia, J.P. Small, D.B. Farmer, and P. Avouris, Nano Lett., 9 (2009), 422.

[35] R. Grantab, V.B. Shenoy, and R.S. Ruoff, Science 330 (2010), 946.

[36] F.S. Khokhar, R. van Gastel, and B. Poelsema, Phys. Rev. B 82 (2010), 205409.

[37] R.M. Tromp and J.B. Hannon, Phys. Rev. Lett. 102 (2009), 106104.

[38] R. van Gastel, A.T. N'Diaye, D. Wall, J. Coraux, C. Busse, N.M. Buckanie, F. Meyer-zu-Heringdorf, M.H. von Hoegen, T. Michely, and B. Poelsema, Appl. Phys. Lett. 95 (2009), 121906.

[39] C. Heininger, L. Kampschulte, W.M. Heckl, and M. Lackinger, Langmuir 25 (2009), 968.

[40] R.F. Egerton, P. Li, and M. Malac, Micron 35 (2004), 399.

[41] J.V. Barth, J. Weckesser, C. Cai, P. Gunter, L. Burgi, O. Jeandupeux, and K. Kern, Angew. Chem. 112 (2000), 1285.

[42] S. Stepanow, N. Lin, F. Vidal, A. Landa, M. Ruben, J.V. Barth, and K. Kern, Nano lett. 5 (2005), 901.

[43] N. Lin, A. Dmitriev, J. Weckesser, J.V. Barth, and K. Kern, Angew. Chem. Int. Ed. 41 (2002), 4779.

[44] S. Stepanow, T. Strunskus, M. Lingenfelder, A. Dmitiriev, H. Spillmann, N. Lin, J.V. Barth, C. Woll, and K. Kern, J. Phys. Chem. B 108 (2004), 19392. 


\section{Experimental method}

This chapter gives an introduction to the growth methods and the characterization techniques employed in this work. A brief description of LEEM is given, including the more common image contrast mechanisms and characterization capabilities of LEEM, i.e. real-time imaging, microdiffraction, and photoemission. The instrumentation used for the experiments is described with common operational conditions. In the later part of the chapter, the sample preparation methods are described. 


\subsection{Low Energy Electron Microscopy (LEEM)}

In this section, we will briefly discuss the Low Energy Electron Microscopy (LEEM) [1], the instrument, its contrast mechanisms, and some examples of its applications. LEEM utilizes low energy, elastically backscattered electrons to image surfaces with high spatial and temporal resolution. The strong interaction of low energy electrons with matter yields extreme surface sensitivity in LEEM. This makes LEEM a powerful tool to study the static and dynamic properties of surfaces [2] and thin films [3, 4]. Processes like growth and decay [5], phase transitions [6, 7], reactions as well as structure and morphology can all be investigated with LEEM. The following properties give LEEM a unique position in the field of surface image microscopy.

- Real-time imaging capability at video rate

- Several contrast mechanisms for image formation

- Large dynamic range

- Large field of view

- Vertical atomic resolution combined with high lateral resolution

\subsubsection{Contrast mechanisms}

LEEM can be seen as the imaging counterpart of Low Energy Electron Diffraction (LEED). The imaging is achieved by making an angular selection of the diffracted electrons using a mechanical aperture in the imaging column of the instrument. Therefore, the fundamental contrast mechanism is diffraction contrast. The diffraction contrast results from differences in surface and thin film structure or the existence of different surface phases. There are two modes of imaging to exploit the diffraction contrast:

- Bright field mode (specular or (00) LEED spot is used for the imaging)

- Dark field mode (non-specular LEED spot is used for the imaging).

A LEEM bright-field image of a pristine $\mathrm{Cu}(001)$ surface is shown in Fig. 2.1(a). Surface atomic steps appear as dark lines, separating terraces. An example of diffraction contrast and how it is visualized is shown in Fig. 2.1(b). It is a LEEM bright-field image of a graphene surface covered with domains consisting of 4,4'-biphenyldicarboxylic acid (BDA) molecules. The bright areas are the graphene surface and the dark areas are the BDA domains. The structural difference between the two types of surface leads to bright field contrast and makes it possible to observe the adsorption and growth of BDA molecular domains on graphene. In other words, different structures give different reflectivities at a given electron energy, the so-called I(V) curve. A reflectivity difference leads to contrast in LEEM images as shown in Fig. 2.2. This is the most commonly used mode of imaging in LEEM. LEEM bright field intensity has been used in a novel way such as to determine the adatom concentration on surfaces [8,9]. All the deposition experiments described in this thesis were performed in the bright-field imaging mode while dark-field LEEM was employed to study the thin film structure when 

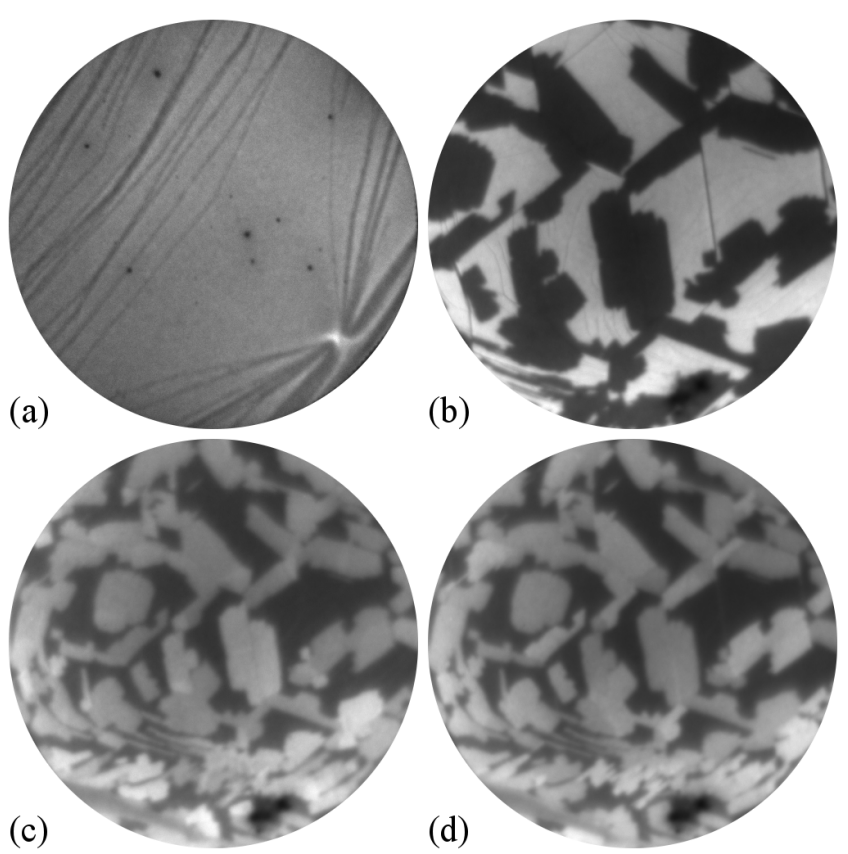

Figure 2.1:

(d)

(a) A pristine $\mathrm{Cu}(001)$ surface. Surface atomic steps appear as dark lines, separating terraces. The dark spots are defects in the detector. The field of view (FoV) is $3 \mu \mathrm{m}$. (b) A bright field LEEM image of BDA domains on graphene. Bright areas represent the graphene surface and dark areas are BDA domains. (c, d) Dark field LEEM images acquired in the same area as (b) but using the superstructure LEED spots of the BDA domains. The graphene surface now appears dark and BDA areas that contribute to the diffracted intensity of the superstructure spots appear bright. A variation of the gray scale is observed between panels $(c)$ and $(d)$ when the aperture is repositioned, illustrating the sensitivity of the image contrast to the momentum transfer parallel to the surface. FoV is $10 \mu \mathrm{m}$ and electron energy is $5.9 \mathrm{eV}$.

the films consisted of several rotational domains. LEEM images shown in Figs. 2.1(c) and (d) were recorded in dark-field mode.

A second important contrast mechanism is the so-called phase contrast, which makes it possible to observe the atomic steps on a certain surface and provides a possibility to achieve atomic resolution in the vertical direction in LEEM. The contrast arises from the interference of electron waves that are reflected from terraces on opposite sides of a step. At certain electron energies, destructive interference occurs between electron waves from the two adjacent terraces at the position of a step on the surface. This is why a step appears as a dark line in a LEEM image as shown in Fig. 2.1(a). The dark lines are the atomic steps on the clean $\mathrm{Cu}(001)$ surface.

Local work function differences and topography variations on the surface can also generate contrast in LEEM. The variations in the accelerating field of the instrument are most prominently seen when a LEEM is used in the mirror mode. In this mode of operation, the sample bias is adjusted so that the electrons reflect in front of the sample 


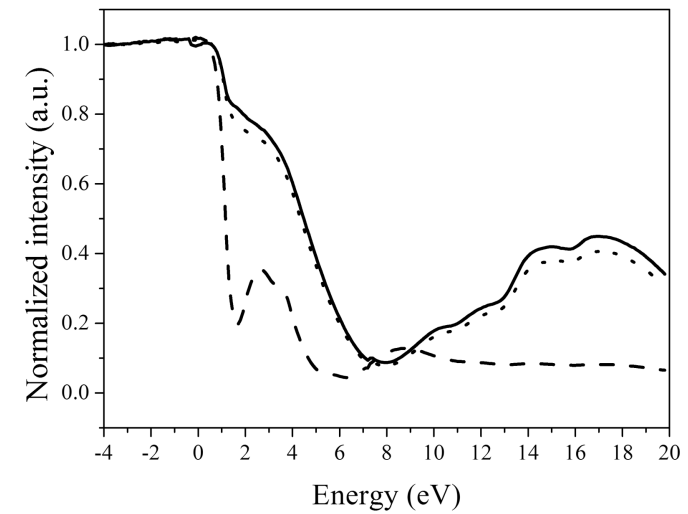

Figure 2.2:

$I(V)$ curves for graphene (solid line), BDA admolecules on graphene (dotted line) and BDA domains on graphene (dashed line).

without interacting or interacting only very weakly with the surface. At these very low electron energies, the reflected intensity becomes extremely sensitive to potential differences and field distortions produced by the topographic features.

\subsection{Low Energy Electron microDiffraction ( $\mu$ LEED)}

LEED is an integral part of LEEM. In this work, LEED is used to determine the crystal quality and cleanliness of the samples before the deposition of organic molecules. After the deposition and growth of organic structures on the samples, Low Energy Electron microDiffraction ( $\mu$ LEED) was employed to determine the structure of a small area such as a terrace or a domain. $\mu$ LEED employs a field-limiting aperture to select an area of interest on the sample to carry out LEED measurements. In the LEEM instrument, it is possible to select a sample area as small as $1.4 \mu \mathrm{m}$ in diameter which is the most frequently used aperture for $\mu$ LEED measurements described in this thesis.

\subsection{PhotoEmission Electron Microscopy (PEEM)}

In PhotoEmission Electron Microscopy (PEEM), electrons emitted from a sample in response to the absorption of ionizing radiation are used to form an image. In our case, we use ultraviolet (UV) light produced by a Hg discharge lamp to perform threshold-PEEM. The dominant wavelength of the photons produced by the discharge is $253.7 \mathrm{~nm}$ or $4.89 \mathrm{eV}$. This value of the photon energy is at or close to the work function of most materials. The yield of emitted electrons is directly determined by the ionization cross-section of the material for that photon energy. To first order, the main mechanism of image contrast in the threshold-PEEM is therefore the sample work function where regions with a low work function will yield higher intensities. Differences in the local work function result in the image contrast. The spatial resolution of PEEM is not as good as that of LEEM, however, it allows us to quantitatively measure work 


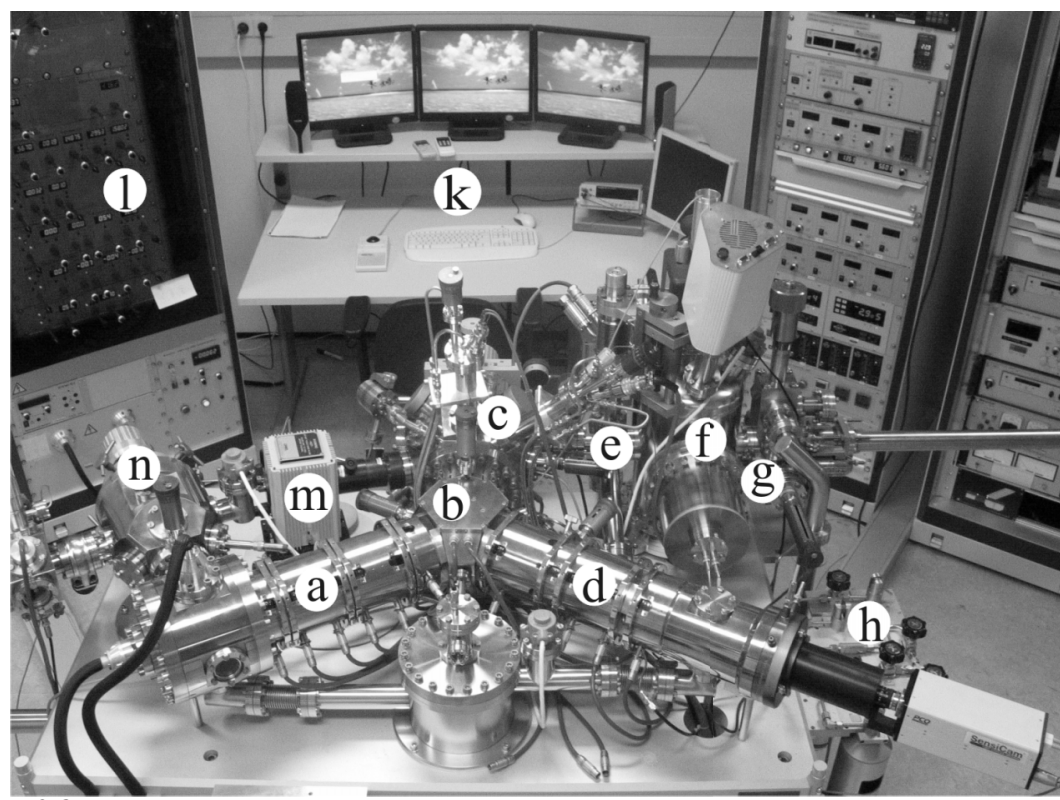

Figure 2.3:

SPLEEM instrument at the Physics of Interfaces and Nanomaterials group. Different components of the LEEM are highlighted by letters. (a) Illumination column. (b) Beam separator. (c) Main chamber. (d) Imaging column. (e) Auger Electron Spectroscopy. (f) Sample preparation chamber. (g) Parking space for 5 samples. (h) Control panel for gases used in sample preparation. (k) Control panel for LEEM. (l) LEEM Electronics. (m) UV Lamp for PEEM. (n) Chamber for the generation of spin polarized electrons. A detailed description on these parts is given in section 2.4 .

function variations on surfaces.

\subsection{Instrumentation}

Fig. 2.3 shows a photograph of the spin-polarized LEEM (SPLEEM) at the Solid State Physics (now known as Physics of Interfaces and Nanomaterials) group of University of Twente and which was used to perform the experimental work described in this thesis. The instrument is unique in the sense that it integrates the capabilities of (1) PEEM, (2) LEEM, and (3) magnetic sample imaging (SPLEEM) in one setup. In this project, we exclusively work with LEEM and PEEM. The structure of the LEEM system can be divided into three major parts: (1) the illumination (a) and imaging columns (d), (2) the main chamber with sample manipulator (c), and (3) the sample preparation chamber (f) which is equipped with a sputtering and annealing facility, Auger Electron Spectroscopy (AES) (e), mass spectrometer, parking space for 5 samples (g), and with a loadlock. The imaging and illumination columns are connected to the main chamber through a gate valve. The illumination column consists of $\mathrm{a} \mathrm{LaB}_{6}$ electron gun with a Wehnelt electrode for controlling the electron emission, magnetic condensor lenses with magnetic deflection coils, and an illumination aperture manipulator having three 
apertures allowing for a reduction of the size of the beam spot on the sample surface to $19 \mu \mathrm{m}, 4.8 \mu \mathrm{m}$, and $1.4 \mu \mathrm{m}$, respectively. The imaging column uses magnetic lenses for image magnification, magnetic deflectors and stigmators, three contrast apertures $(100,30$, and $10 \mu \mathrm{m})$, and a set of microchannel plates with a fluorescent screen to project the diffraction patterns and LEEM images. A high resolution CCD camera acquires images from the fluorescent screen at a video rate or slower as desired for the purpose of signal acquisition. The beam separator (magnetic prism) (b) deflects the electron beam over an angle of $60^{\circ}$ and is part of both the illumination and imaging columns. The microscope objective lens is placed in the main chamber. The sample is biased with respect to the objective lens to create the field that is required to decelerate the electrons to an energy of a few $\mathrm{eV}$. In the main chamber, samples can be annealed and cooled down with liquid nitrogen flow. Sample translational motion and sample tilt adjustment is achieved with a pair of translating micromanipulator screws. Further detailed description of the instrument and its capabilities can be found in ref. [1].

The sample preparation chamber was further developed to improve the working conditions during sample preparation. The standard chamber was replaced with the dome shaped chamber shown in Fig. 2.3. It has a quadrupole mass spectrometer for the residual gas analysis, parking space for five samples, and allows AES analysis in order to determine the amount and chemical nature of contaminants on the sample. A $400 \mathrm{l} / \mathrm{s}$ magnetic turbo pump was installed to achieve a suitable base pressure of $1 \times 10^{-10} \mathrm{mbar}$. The preparation chamber is also equipped with two separate inlets for gas treatment of a sample.

\subsection{Sample preparation}

Preparation of a sample surface to typical LEEM requirements, i.e. contamination free large terraces of several microns wide, bounded by surface steps that do not have any visible pinning sites, is a challenging issue. It becomes an even more challenging issue when the organic adsorbates deposited in the experiments themselves act as contamination source that prevent recycling of the metal crystals using traditional approach of sputtering and annealing. In the following paragraphs, we describe different methods used to prepare the $\mathrm{Cu}(001), \operatorname{Ir}(111)$, and graphene substrates for our experiments.

\subsection{1 $\mathrm{Cu}(001)$ surface preparation}

Prior to insertion into the vacuum system, the $\mathrm{Cu}(001)$ oriented crystal was annealed at $950^{\circ} \mathrm{C}$ in a $\mathrm{H}_{2} / \mathrm{Ar}$ gas mixture for 48 hours in order to reduce the bulk sulfur content of the crystal. After insertion into the vacuum, the common procedure of sputtering and annealing in ultra high vacuum was employed. This method produced clean areas on certain parts of the $\mathrm{Cu}$ surface, however, the distribution of contaminants on the surface was very inhomogeneous. Fig. 2.4(a) is a LEEM image of a $\mathrm{Cu}(001)$ surface after a few cycles of sputtering and annealing. The black dots are believed to be the remnants of the diamond polishing paste used to polish the surface. We found this to be one of the main sources of contamination on the surface in the initial stages of preparation of the surface, prior to the deposition experiments. Removing these contaminants proved to be time-consuming as it essentially requires the complete removal 


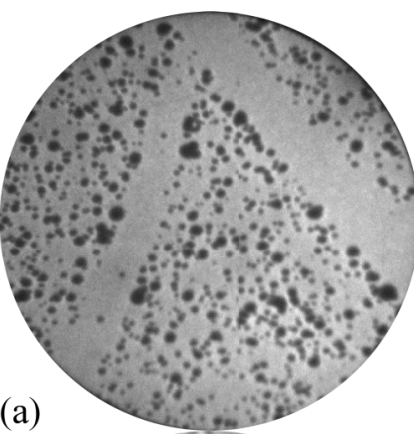

(a)

Figure 2.4:

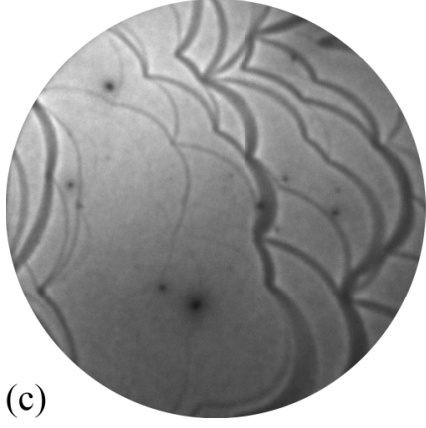

(b)
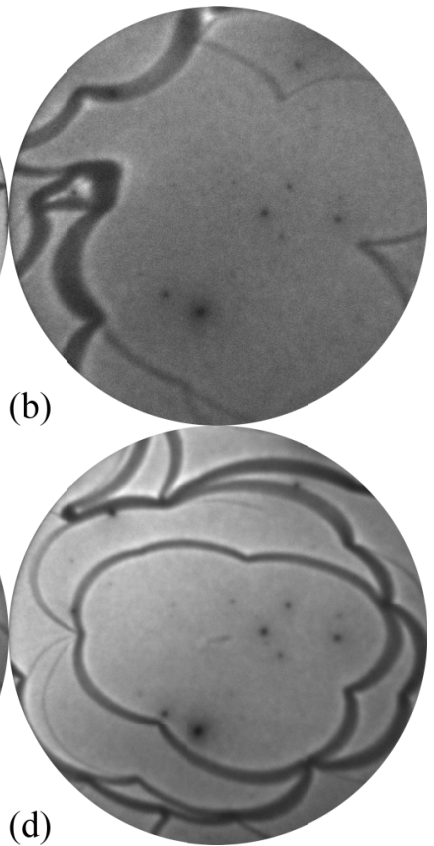

(a) A pristine $\mathrm{Cu}(001)$ surface in the early stages of preparation. The black dots are believed to be remnants of diamond polishing paste. FoV is $10 \mu \mathrm{m}$. (b) $\mathrm{Cu}(001)$ surface after more than 100 cycles of $\mathrm{Ar}^{+}$ion sputtering and annealing, FoV is $3 \mu \mathrm{m}$. (c) and (d) are images of a clean $\mathrm{Cu}(001)$ surface after 3 cycles of $\mathrm{Ar}^{+}$ion sputtering and annealing in the $\mathrm{H}_{2}$ environment. FoV is $3 \mu \mathrm{m}$ and $5 \mu \mathrm{m}$, respectively.

of the $0.25 \mu \mathrm{m}$ diamond polishing grains by sputtering. Fig. 2.4(b) shows the end result of this procedure. To reduce the preparation time between different deposition experiments, a number of different methods were tried:

- Exposure of $\mathrm{Cu}(001)$ to $1 \times 10^{-7}$ mbar of $\mathrm{O}_{2}$ pressure at annealing temperature for $40 \mathrm{~s}$.

- Electrochemical etching of $\mathrm{Cu}(001)$ surface in $\mathrm{H}_{3} \mathrm{SO}_{4}$ solution.

- $\mathrm{Ar}^{+}$ion sputtering at elevated surface temperature.

- $\mathrm{Ar}^{+}$ion sputtering and annealing in a $\mathrm{H}_{2}$ pressure of $1 \times 10^{-6}$ mbar.

However, out of these methods, $\mathrm{Ar}^{+}$sputtering and annealing in $\mathrm{H}_{2}$ pressure met our requirements for producing clean $\mathrm{Cu}(001)$ surfaces for our LEEM experiments in an acceptable time as illustrated in Figs. 2.4(c) and 2.4(d).

\subsection{2 $\operatorname{Ir}(111)$ surface preparation}

Iridium surfaces are commonly cleaned by annealing at elevated temperatures in oxygen [10]. Carbon is found to be the main source of contaminations on $\operatorname{Ir}(111)$ 


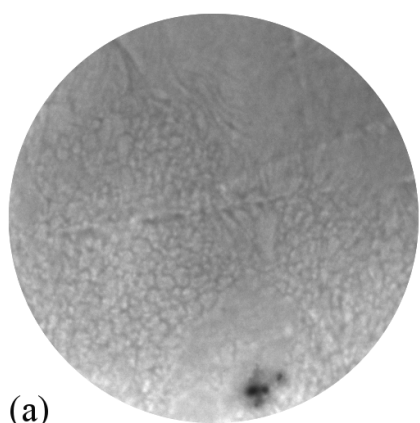

(a)

(c)

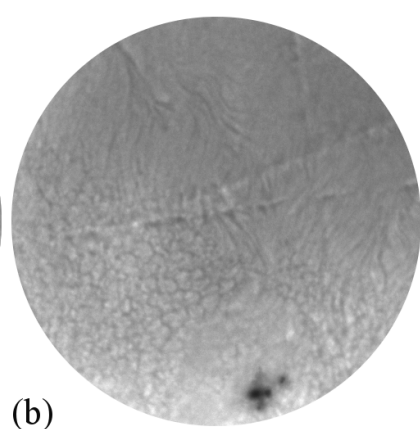

(b)

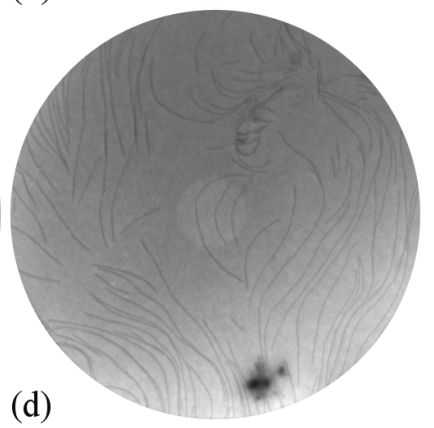

Figure 2.5:

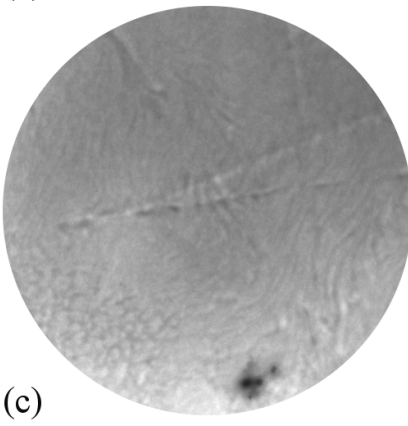

(a-c) A series of LEEM images obtained at a FoV of $25 \mu \mathrm{m}$ with electron energy of $2.4 \mathrm{eV}$ at a surface temperature of $875 \mathrm{~K}$ and $\mathrm{O}_{2}$ exposure of $1 \times 10^{-7}$ mbar. The images illustrate the cleaning process of the Ir(111) surface. Times indicated for the panels are measured with respect to panel $(a)$. $\quad(\mathbf{b}, \mathbf{t}=\mathbf{3 6} \mathbf{s})$ and $(\mathbf{c}, \mathbf{t}=96 \mathbf{s})$ show that the surface contaminations are rapidly removed from the surface. $(\mathbf{d}, \mathbf{t}=\mathbf{1 6 9} \mathbf{s})$ The clean $\operatorname{Ir}(111)$ surface with atomic steps that is acquired after the $\mathrm{O}_{2}$ treatment. The image is obtained at $10 \mu \mathrm{m}$ FoV with an electron energy of $2.7 \mathrm{eV}$.

surface which can easily be cleaned by annealing at elevated temperatures in $\mathrm{O}_{2}$ environment. Thus, constitutes a favorable choice of substrate to perform experiments with organic molecules. In some cases, sputtering was required prior to annealing when Iridium surface is fully covered with carbon. Sputtering creates patches of clean Iridium where $\mathrm{O}_{2}$ can adsorb and react to remove contamination. In our experiments, an $\operatorname{Ir}(111)$ surface is first exposed to an $\mathrm{O}_{2}$ pressure of $1 \times 10^{-7}$ mbar in the instrument's main chamber and is annealed to an elevated temperature. This cleaning procedure is monitored in-situ by LEEM as shown in Figs. 2.5(a)-(c). The $\mathrm{O}_{2}$ exposure is halted as soon as a clean surface is observed as shown in Fig. 2.5(d).

\subsubsection{Graphene preparation}

Graphene films were prepared by Chemical Vapor Deposition (CVD) of Ethylene $\left(\mathrm{C}_{2} \mathrm{H}_{4}\right)$ on the $\operatorname{Ir}(111)$ surface at a temperature of $875 \mathrm{~K}$ [11]. Prior to the growth of graphene, the $\operatorname{Ir}(111)$ surface is cleaned following the procedure described in section 2.5.2. After that, it is exposed to an $\mathrm{C}_{2} \mathrm{H}_{4}$ pressure of $1 \times 10^{-8}$ mbar. The $\mathrm{C}_{2} \mathrm{H}_{4}$ adsorbs on the $\operatorname{Ir}(111)$ and instantly decomposes into its atomic constituents, Carbon 
and Hydrogen. The Hydrogen rapidly desorbs from the surface leaving mobile Carbon adatoms to form graphene. The growth of graphene sheets was followed in real-time using PEEM until sufficiently large flakes were formed as shown in Fig. 2.6. This
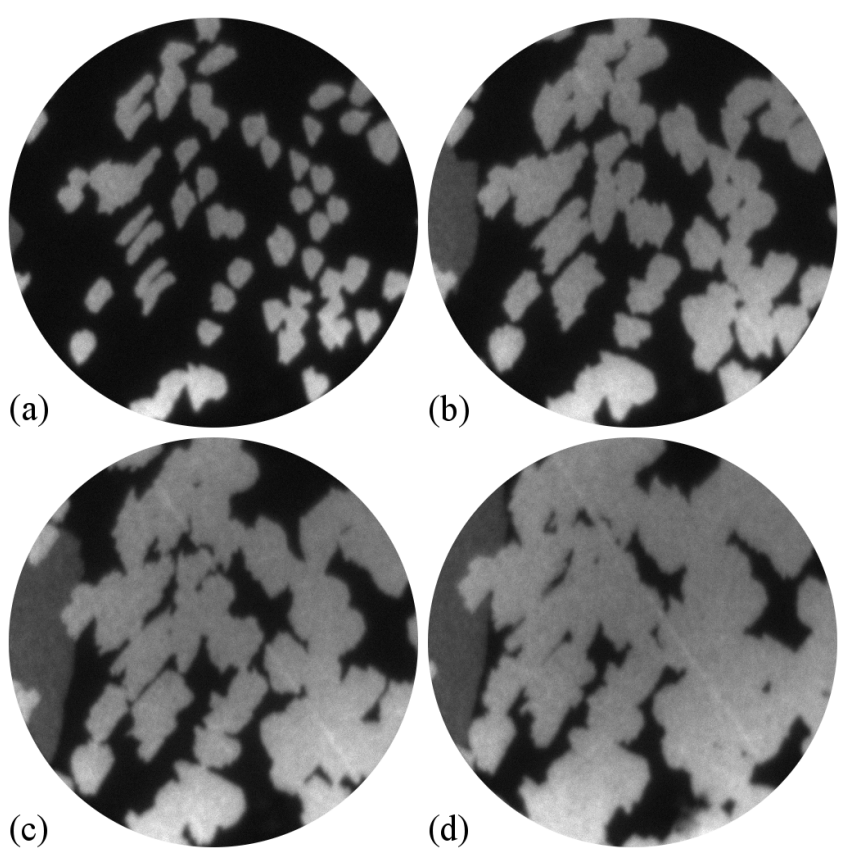

Figure 2.6:

(d)

A series of PEEM images obtained at a FoV of $100 \mu \mathrm{m}$ illustrating the growth of graphene sheets. Times indicated for the panels are measured with respect to the start of $\mathrm{C}_{2} \mathrm{H}_{4}$ adsorption. $(\mathbf{a}, \mathbf{t}=\mathbf{2 0 5} \mathbf{s})$ Graphene domains (light grey) nucleate on the $\operatorname{Ir}(111)$ surface (dark black background). ( $(\mathbf{b}, \mathbf{t}=\mathbf{4 1 0} \mathrm{s})$ With added $\mathrm{C}_{2} \mathrm{H}_{4}$ adsorption, graphene domains grow further and coalesce. Nucleation of graphene domains that are rotated (dark grey) with respect to the Ir(111) surface is also observed. $(\mathbf{c}, \mathbf{t}=\mathbf{6 2 0} \mathbf{s})$ Both rotational graphene domains grow in size with $\mathrm{C}_{2} \mathrm{H}_{4}$ deposition. $(\mathbf{d}, \mathbf{t}=920 \mathrm{~s})$ Graphene domains further grow and coalesce to cover most of the Ir(111) surface.

growth procedure yields large monolayer sheets of graphene that cover extended areas of the surface. The graphene sheets that are grown in this way constitute ideal substrates to study the behavior of organic molecules on graphene since the growth mode of the graphene sheets intrinsically limits the thickness of the sheets to a single monolayer. 


\section{Bibliography}

[1] E. Bauer, Surf. Rev. Lett. 5 (1998), 1275.

[2] F. Watanabe, S. Kodambaka, W. Swiech, J.E. Greene, and D.G. Cahill, Surf. Sci. 572 (2004), 425.

[3] E.Z. Luo, Q. Cai, W.F. Chung, B.G. Orr, and M.S. Altman, Phy. Rev. B Condens. Matter. 54 (1996), 14673.

[4] A. Al-Mahboob, J.T. Sadowski, T. Nishihara, Y. Fujikawa, Q.K. Xue, K. Nakajima, and T. Sakurai, Surf. Sci. 601 (2007), 1304.

[5] J.B. Hannon, H. Hibino, N.C. Bartelt, B.S. Swartzentruber, T. Ogino, and G.L. Kellogg, Nature 45 (2000), 552.

[6] W. Telieps, Appl. Phys. A-Mater. 44 (1987), 55.

[7] K.F. McCarty and N.C. Bartelt, Surf. Sci. 540 (2003), 157.

[8] J. de la Figuera, N.C. Bartelt, and K.F. McCarty, Surf. Sci. 600 (2006), 4062.

[9] E. Loginova, N.C. Bartelt, P.J. Feibelman, and K.F. McCarty, New J. Phys. 10 (2008), 093026.

[10] R.G. Musket, W. McLean, C.A. Colmenares, D.M. Makowiecki, and W.J. Siekhaus, Appl. Surf. Sci. 10 (1982), 143.

[11] J. Coraux, A.T. N'Diaye, M. Engler, C. Busse, D. Wall, N. Buckanie, F.-J. Meyer zu Heringdorf, R. van Gastel, B. Poelsema, and T. Michely, New J. Phys. 11 (2009), 023006. 
Domain patterns and two-dimensional self-assembly

This chapter provides a brief introduction to the formation of domain patterns in twodimensions and the role of competing interactions in inorganic material systems as well as surface supported organic films used in this work. Initial results obtained with trimesic acid (TMA) molecules are outlined and the role of the imaging electrons in causing possible radiation damage to the organic thin films is also discussed. 


\subsection{Domain patterns: Two-dimensional self-assembly and competing interactions}

Self-assembly is a term used to describe processes in which a disordered system of pre-existing components forms an organized structure or pattern as a consequence of specific interactions among the components themselves, without external direction [2]. It is schematically depicted in Fig. 3.1.
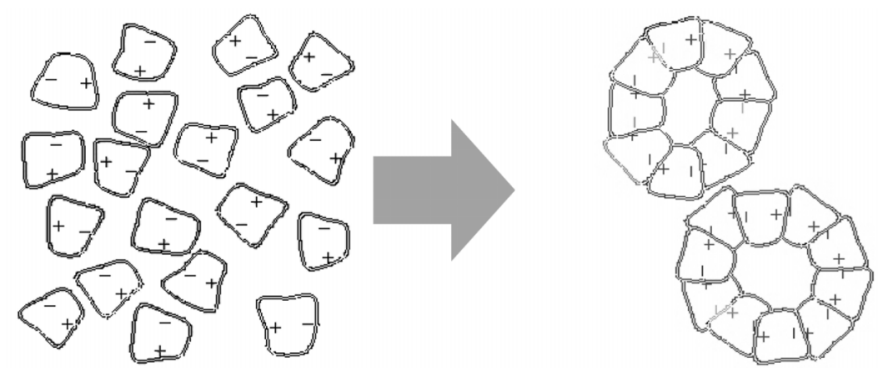

Figure 3.1:

Self-assembly of individual components through a driving interaction [1].

There are two types of self-assembly processes: static and dynamic. In static selfassembly, the system is in local or global equilibrium and it does not dissipate energy. Atomic, ionic, and molecular crystals are common examples of static self-assembly. In the other case, the system dissipates energy. The energy dissipation occurs because of the interactions between components of the self-assembled patterns. Self-assembled systems can be found in biological, chemical, and physical fields of science [2].

In nanoscience, self-assembly is being investigated as an alternative to the existing top-down approach to form nanometer sized structures. Top-down methods become increasingly expensive as the size of the final structures decreases. They are also time consuming. In bottom-up methods, i.e. self-assembly, very small components organize into structures with nanodimensions. It occurs in 'no time' and in a very cost effective manner. Self-assembly, however, poses a challenge and that challenge is to attain sufficient control over the final size of a self-assembled structure. In what follows, we will describe how this challenge is tackled in two dimensions, conceptually and experimentally.

The first question that needs to be asked is why the growth of two-dimensional nanosized structures is so important? The answer to this question is twofold: it is relevant to understand the underlying fundamental physics as well as to harnass the potential of nanosized structures to be used in template applications. It is complicated, however, by the fact that for two-dimensional systems, short-range attractive interactions betweens atoms and molecules tend to dominate. The high perimeter to area ratio of ordered small domains also makes them thermodynamically unstable [3]. Longerranged repulsive forces can act as a stabilizing force. Their existence can lead to a competion with the short-ranged attractive interactions. The resulting stabilization can lead to the formation of macroscopic self-assembled domains. The domains are not 

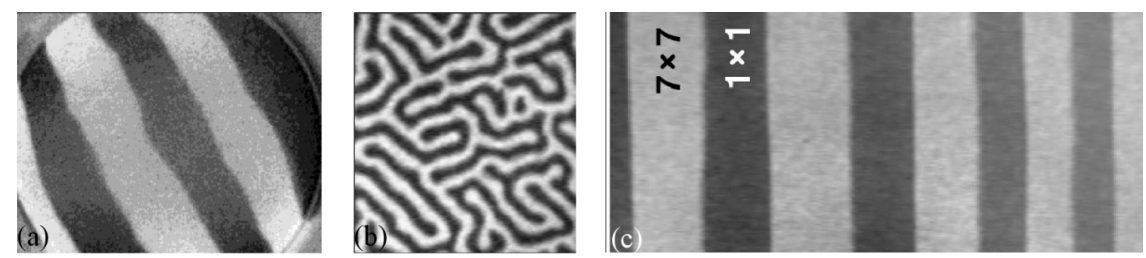

Figure 3.2:

(a) Domain pattern formation in the $\mathrm{Fe} / \mathrm{Cu}(100)$ system. The FoV equals $7 \mu \mathrm{m}$. Contrast in the SPLEEM image comes from the orientation of the local magnetization vector. Bright and dark regions are magnetized up and down, respectively [6]. (b) Self-assembled domain pattern in the Pb/Cu(111)system. FoV is $1.7 \mu \mathrm{m}$. Two different surface phases appear dark and bright, respectively. The stabilizing force is of an elastic nature [7]. (c) LEEM image of coexisting Si $(111)-(7 \times 7)$ and $(1 \times 1)$ domains. FoV is $1 \mu \mathrm{m}$. Elastic interactions give rise to phase coexistence around the $(7 \times 7)$ to $(1 \times 1)$ phase transition temperature [5].

only thermodynamically stable, but tailoring of the balance between the interactions allows us to control the feature size [4]. This opens up a whole new field of research: competing interactions and two-dimensional self-assembly. The long-range interactions can have various physical origins, e.g. elasticity, electrostatics or magnetism. Temperature, the strength of the forces, the nature of the surface, and coverage are the most important parameters, which influence the details of a surface domain pattern. In other words, these parameters can be used to tune the domain patterns.

Advancements in electron microscopy in recent decades have made it possible to investigate the dynamics of domain patterns in great detail. Several theoretical predictions have been confirmed experimentally. Particularly, patterns formed on metal and semiconductor surfaces are well understood [5-7]. In magnetic systems, competion between short-ranged exchange interactions and long-ranged dipolar interactions gives rise to the formation of magnetic domains. Although the dipolar interactions are weak, they become significant when large numbers of dipoles are involved. This enables them to compete with the exchange interaction [4]. An example is shown in Fig. 3.2(a). It is a SPLEEM image of Iron $(\mathrm{Fe})$ domains on $\mathrm{Cu}(001)$. The stability of the magnetic stripe domains is attributed to competing interactions on a different length scale. Domain patterns like those in Figs. 3.2(b) and 3.2(c) show that despite the fundamentally different nature of the interactions, similar patterns are observed.

For elastic relaxations, theoretical studies predict the formation and stabilization of periodic structures with well-defined equilibrium sizes [8-10]. If we consider a surface with two different phases, A and B, the long-range elastic interactions between phase boundaries originate from different surface stresses of the two phases. The elastic relaxations that occur at domain (phase) boundaries not only stabilize the pattern, but also play a major role in its size selection. The energy $\Delta E$ associated with the formation of the domain pattern can be written in the following way [10],

$$
\Delta E=-\frac{C_{\text {elas }}}{l} \ln \left(\frac{l}{\pi a} \sin (\pi f)\right),
$$


where $l$ equals the average size of the domains, $f$ is the relative area fraction of one of the two phases and $a$ is a microscopic cutoff length. The parameter $C_{\text {elas }}$, which gives the magnitude of elastic interactions, is given by

$$
C_{\text {elas }}=\frac{(\Delta \sigma)^{2}\left(1-\nu^{2}\right)}{\pi E},
$$

where $E$ and $\nu$ are the Young's modulus and Poisson ratio of the substrate, and

$$
\Delta \sigma=\sigma_{A}-\sigma_{B}
$$

is the difference in the normal components of the surface stress between A and B domains. The equilibrium feature size $l_{0}$ is determined by balancing the elastic energy against the energetic cost, $F_{b}$ of creating boundaries.

$$
l_{0}=\pi a \csc (\pi f) \exp \left(\frac{F_{b}}{C_{\text {elas }}}+1\right)
$$

For the two examples shown in Figs.3.2(b) and 3.2(c), the equilibrium size of the observed domains can indeed be altered. By changing temperature, the role of entropy in both systems can be enhanced or reduced, leading to a change in the balance between $F_{b}$ and $C_{\text {elas }}[5,7]$.

The theory of domain pattern formation due to competing short-ranged attractive and long-ranged repulsive electrostatic interactions is closely related to the elastic case. Both types of interactions lead to similar behaviour because of their identical $r^{-3}$ scaling [10]. A system that self-assembles into a domain structure due to electrostatic interactions lowers its energy by an amount $\Delta E$ given by

$$
\Delta E=-\frac{C_{\text {elec }}}{l} \ln \left(\frac{l}{\pi a} \sin (\pi f)\right)
$$

It is similar to the elastic case with the exception that the factor $C$ in the electrostatic case is given by

$$
C_{\text {elec }}=\frac{1}{8 \pi^{2}}(\Delta \phi)^{2}
$$

However, it is worthwhile to note that elastic interactions are more complicated because of their tensor nature. For a realistic work function difference of $\Delta \phi=1 \mathrm{eV}, C_{\text {elec }}=$ 0.87 meV/Åimplying that a domain boundary energy no more than a few meV/Å is allowable if the domain pattern is to remain observable [10]. An example is shown in Fig. 3.2(c). In that case, both elastic $(0.28 \mathrm{meV} / \AA)$ and electrostatic $(0.02 \mathrm{meV} / \AA)$ interactions are present. Although the elastic interactions are clearly dominating, the electrostatic interactions also contribute towards the domain pattern's stability [5]. In most cases, domain patterns extend over many microns and depending on the system feature sizes range from a few nanometers to well over one hundred nanometers [57, 11-20]. 

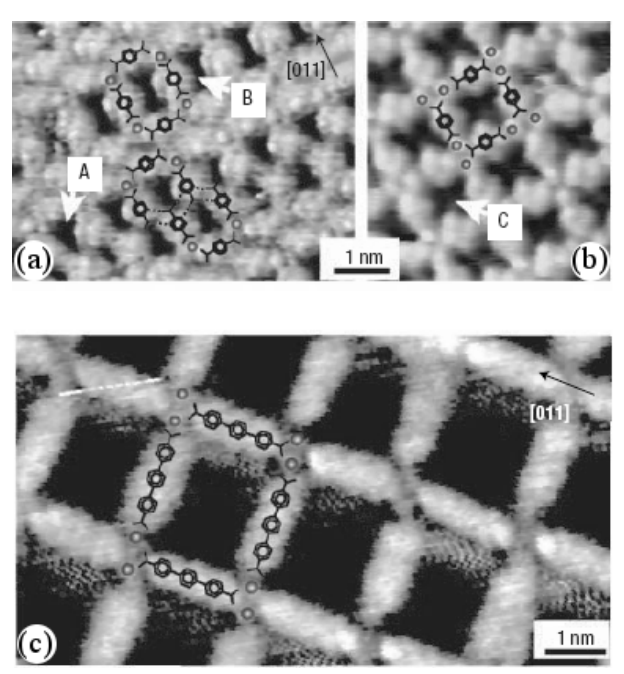

Figure 3.3:

The size of an opening or cavity in a self-assembled structure can be tuned by selecting different length of molecules. STM images of (terephthalic acid) TPA-Fe architectures on the $\mathrm{Cu}(001)$ surface. (a) Structures with two distinct types of nanocavities (marked by A and B). Dashed lines indicated the potential intermolecular hydrogen-bridges. (b) A network of two-dimensional square cavities (marked by $\mathrm{C}$ ). The size of cavities is larger than cavities shown in (a). (c) FeTDA open network with rectangular cavities. Arrows on the images indicate the high-symmetry [011] direction of the $\mathrm{Cu}(100)$ substrate. The size of cavities is larger than the cavities shown in both (a) and (b). Fe atoms are shown as blue spheres [26].

\subsection{Organic molecules and surface supported structures}

Supramolecular chemistry studies the interactions between molecules. The forces that are used to organize and maintain supramolecular self-assemblies in three dimensions, are weak. Bonds typically result from noncovalent interactions such as hydrogen bonds, Van der Waals forces, and metal-organic coordination bonds [21]. The basic concepts of supramolecular chemistry can be applied to two-dimensional self-assembly for surface supported structures. A large number of experimental studies have been performed to grow such two-dimensional nanostructures [22-25]. The formation of two-dimensional organic supramolecular nanostructures on surfaces is a growing area of research [25]. The interest, this field is receiving, is largely because the organic functionalisation of solid surfaces has relevant applications, e.g. in catalysis, sensors, adhesion, corrosion inhibition, molecular recognition, optoelectronics, and lithography [23]. The possibility of tailoring the organic molecules makes this field even more exciting. The properties of the final nanostructure are determined by the individual molecules. An example is shown in Fig. 3.3. The size of the opening or cavity in a self-assembled structure can be altered by simply changing the length of organic molecule. Experimental studies, mostly STM, have shown promising results [22-25]. Another example is shown in Fig. 3.4. Although, these studies highlight the relevance and possibilities for exploiting intermolecular interactions, an in-depth investigation of 


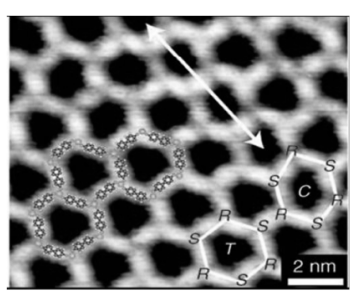

Figure 3.4:

STM images of organic molecular self-assembly. (a) BDA molecules and Fe atoms are codeposited on a Ag(111) surface at room temperature. Admolecules and metal atoms selfassemble into pentagonally shaped cavities. Each Fe adatom is bound to three BDA molecules via metal-organic coordination bonds [29]. (b) BDA molecules on the Au(111) surface at room temperature self-assemble into chains where individual BDA molecules bind head to tail via hydrogen bonds. The image size is $34 \times 34 \mathrm{~nm}^{2}$ [30].

the molecule-surface interaction, underlying long-ranged interactions that drive the formation of organic domains and its influence on the stability of organic nanostructures is still missing.

Metal surfaces will generally show a significant change of work function after the deposition of organic molecules [27]. The relatively weak non-covalent bonding between molecules and the surface on one hand and between the molecules themselves on the other hand, justifies the expectation of self-assembly in these systems. The modification of the surface work function can drive the self-assembly of organic molecules and the resulting structures are stabilized by competition between the potential differences and the energetic cost to form domain boundaries as described in section 3.1. In addition, a study that is performed in-situ and with tailorable molecules, will yield the opportunity to exert control over the size selection of the features. The latter is not only critical for technological applications in which we would like to spread organic structures over large areas but also for the basic understanding of the self-assembly mechanism. The measurement of the strength of the stabilizing interactions, the structures that the organic molecules form as a result and the dynamics that lead to the formation of patterns are the main topics of this thesis.

To realize our study of self-assembled structures on a relevant length scale and investigate the driving forces, an instrument is needed which has a large field of view (FoV) and high spatial resolution. LEEM discussed in Chapter 2 is such a tool. Its real-time imaging capabilities and large FoV that extends to over $100 \mu \mathrm{m}$ enables us to gain the necessary insight into a variety of dynamical processes on surfaces [7] .

\subsection{Growth of TMA structures}

The first task at hand is to select suitable organic molecules to work with and form the self-assembled structures. A promising class of candidates to study self-assembled supramolecular nanostructures is that of planar molecules comprising functional end groups and extended aromatic $\pi-\pi$ systems. Because of the phenyl rings, the molecules tend to bond to most surfaces in a flat-lying geometry. The functional end groups at the molecular periphery are responsible for the intermolecular interactions [28]. They 
Figure 3.5:

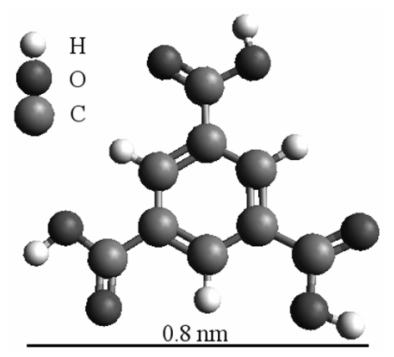

The TMA molecule comprises of a phenyl ring and three identical carboxyl endgroups lying in the same plane.

provide the possibility to benefit from directional bonding, e.g. through hydrogen bonding. These organic species have been successfully employed on surfaces to form large-scale structures [23]. TMA, 1,3,5-benzenetricarboxylic acid, $\mathrm{C}_{3} \mathrm{H}_{3}(\mathrm{COOH})_{3}$ is one such molecule, shown in Fig. 3.5. It is a prototype material for surface supported supramolecular self-assemblies. The molecule is flat, polyfunctional, and 3-fold symmetric, comprising a phenyl ring and three identical carboxyl endgroups in the same plane. TMA is known to assemble in various supramolecular structures due to its trigonal exodentate functionality. The most common motif identified is a planar honeycomb network structure that is formed through the dimerization of the carboxyl groups [23]. The $\mathrm{Cu}(001)$ single crystal surface was employed as a substrate. It was chosen because of its simple surface symmetry. Previous studies of TMA on metal surfaces have yielded information on different interactions (intermolecular, molecule-metal atom, and molecule-surface) within a domain [23, 31]. However, due to instrumental limitations, these studies were unable to yield any insight in the growth dynamics of the domains and any long-range order the domains may exhibit. LEEM with its large FoV and real-time imaging capabilities overcomes these instrumental shortcomings. In the following sub-section, we will discuss LEEM observations of the growth of selfassembled TMA structures on $\mathrm{Cu}(001)$ and compare this to previous STM studies.

\subsubsection{Results and observations}

The experiments are divided in two parts, performed at low temperature $(250 \mathrm{~K})$ and performed at room temperature. Commercially available TMA (Acros Organics, USA) in powder form was deposited by organic molecular beam epitaxy (OMBE) from a Knudsen-cell type evaporator. The temperature of the cell was held constant at $190^{\circ} \mathrm{C}$ during deposition. Decomposition of TMA molecules can occur at around $247^{\circ} \mathrm{C}$ [23]. LEEM images were recorded at a time interval of one second, converted into movies, and later analyzed with a home made computer code.

\section{Low temperature measurements}

In the low temperature measurements, the temperature of the $\mathrm{Cu}(001)$ surface was decreased to $250 \mathrm{~K}$. A temporal evaluation of a typical TMA deposition experiment at low temperature is shown in Fig.3.6. In this experiment, the terraces are not as large as in the case of the room temperature measurements. However, the terraces are 

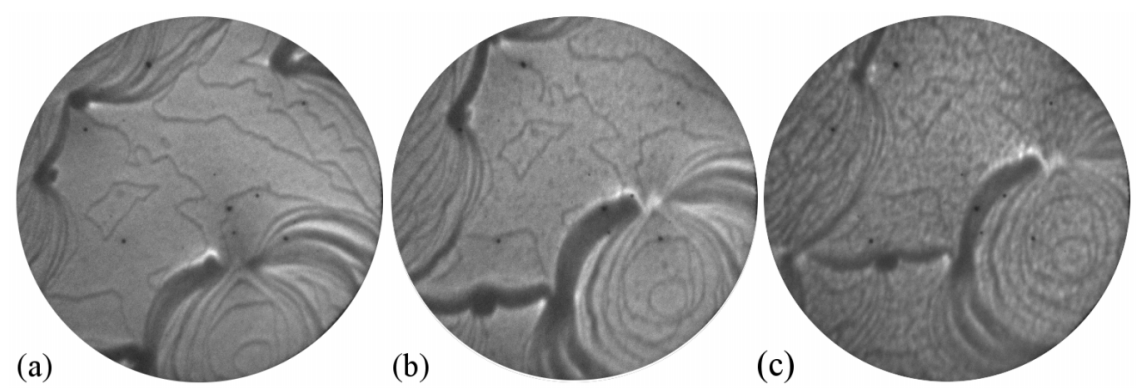

Figure 3.6:

A sequence of deposition and growth of TMA domains obtained at a FoV of $2 \mu \mathrm{m}$ with an electron energy of $2.7 \mathrm{eV} .(\mathbf{a}, \mathbf{t}=\mathbf{0} \mathbf{s})$ Clean $\mathrm{Cu}(001)$ surface, prior to TMA exposure. Atomic steps and pinning sites are present on the surface. $(\mathbf{b}, \mathbf{t}=\mathbf{1 3 7 0} \mathbf{s})$ TMA domains nucleate on the $\mathrm{Cu}(001)$ surface with deposition. Domains nucleate on terraces and atomic steps. The dark features appear in the image are the TMA domains. The domains do not exhibit significant growth. (c, $\mathbf{t}=\mathbf{2 4 7 0} \mathrm{s})$ New domains continue to nucleate on the surface with further deposition of TMA molecules. Existing domains do not show significant mobility.

broad enough to observe the growth dynamics of TMA domains. After a short initial period, the TMA domains nucleate homogeneously and also decorate steps. The TMA domains did not exhibit any mobility throughout the experiment.

\section{Room temperature measurements}

Fig. 3.7 shows a sequence of images obtained during the deposition of TMA on $\mathrm{Cu}(001)$ at room temperature. Fig. 3.7(a) is a typical LEEM image of the clean $\mathrm{Cu}(001)$ surface, prior to TMA deposition. It has one large and a lot of small terraces separated by atomic steps. A large terrace is desired for our LEEM measurements to reduce the influence of surface steps on the growth, especially in a situation where self-assembly is being investigated.

After an initial time during which only the reflected intensity from the surface decreases, deposition of TMA molecules leads to the appearance of dark areas on the surface. We note that the time prior to nucleation is significantly shorter than it is in our low temperature experiments. The dark areas in Fig. 3.7(b) are two-dimensional TMA domains. The TMA domains that form do not exhibit any observable mobility. This suggests a low mobility of isolated TMA molecules on the $\mathrm{Cu}(001)$ surface. Further deposition of TMA, shown in Figs. 3.7(b) to 3.7(e), leads to an increase in average size of the domains. As the growth of TMA approaches one monolayer, domains are observed to coalesce, shown in Fig. 3.7(f). Furthermore, the growing domains appear to form elongated structures.

\subsubsection{Analysis and discussion}

LEEM produces grayscale images. In a grayscale image, the colors are shades of gray (graylevel) and are directly correlated to the electron current density that is projected on the micrchannelplates (MCPs). A common method to analyze grayscale images is thresholding to convert the image into a binary image. The image is segmented 

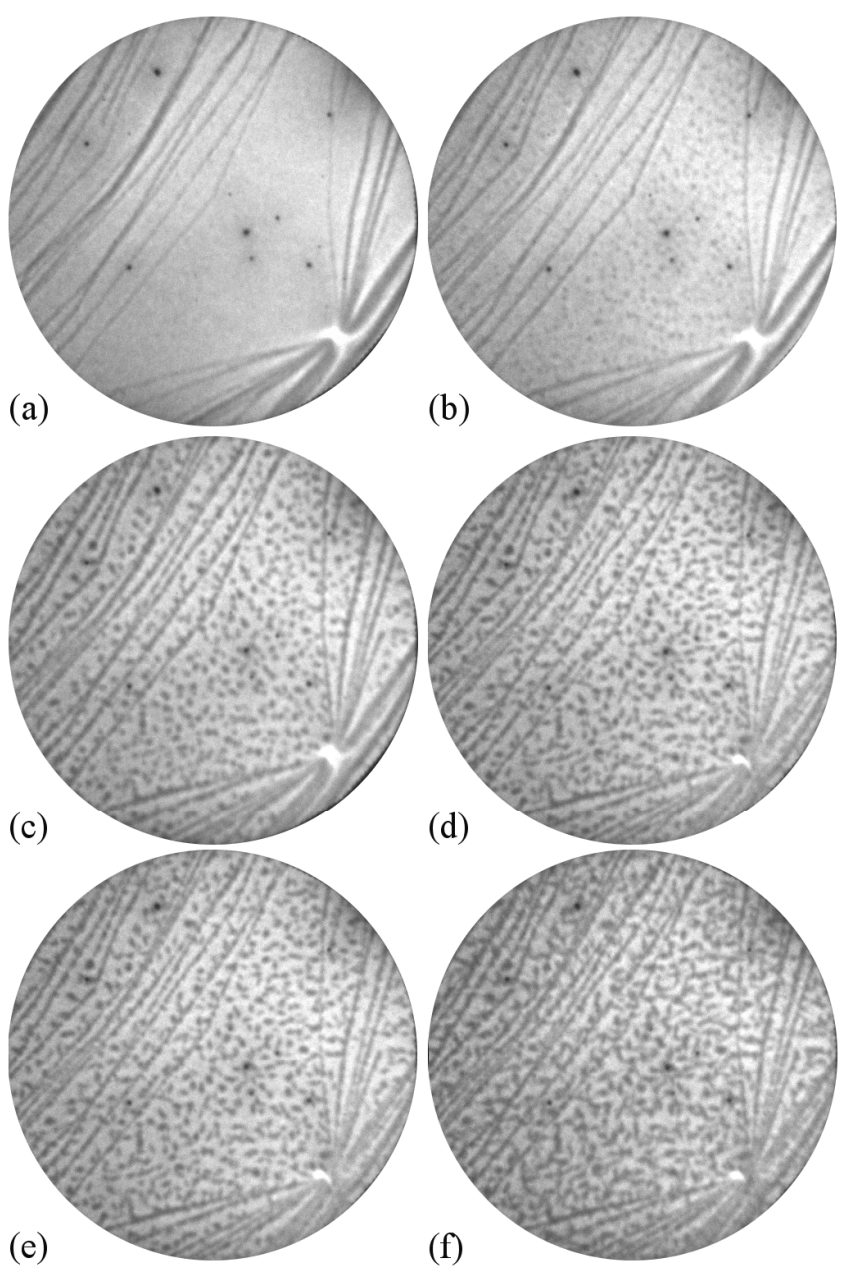

Figure 3.7:

A LEEM image sequence of deposition and growth of TMA domains on $\mathrm{Cu}(001)$ at room temperature obtained at FoV of $2 \mu \mathrm{m}$ with electron energy of $2.7 \mathrm{eV}$. (a, $\mathbf{t}=\mathbf{0} \mathbf{s}) \mathrm{Clean} \mathrm{Cu}(001)$ surface prior to TMA exposure. $(\mathbf{b}, \mathbf{t}=\mathbf{5 0} \mathbf{s})$ TMA domains nucleate on $\mathrm{Cu}(001)$ surface with deposition. Domains nucleate on terraces and atomic steps. $(\mathbf{c}, \mathbf{t}=\mathbf{2 0 0} \mathbf{s})$ Domains grow in size and nucleation of new domains takes place. $(\mathbf{d}, \mathbf{t}=\mathbf{4 0 0} \mathrm{s})$ With continued deposition of TMA, domains grow in size and several coalescence events are observed. $(\mathbf{e}, \mathbf{t}=630 \mathrm{~s})$ TMA domains further increase in size. They do not exhibit any mobility. $(\mathbf{f}, \mathbf{t}=\mathbf{7 0 0} \mathrm{s})$ In the last stages of deposition, mostly coalescence of domains is observed, resulting in elongated TMA structures. 


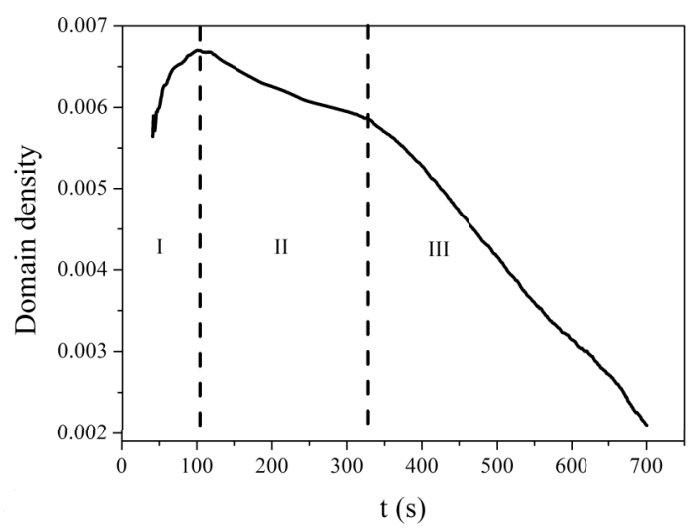

Figure 3.8:

The time dependence of the TMA domain density at room temperature is analyzed. The curve is divided into three regions to understand the behavior of the domain density during deposition. The regimes can be classified as nucleation (I), growth (II), and coalescence (III).

based using a threshold greylevel and split into sections containing domains (dark, 0) and background (bright, 1). The segmentation of a LEEM image into a binary image provides a convenient way to analyze domain properties such as the area, location or boundary length. There should be sufficient contrast, i.e. difference in graylevel, between two segments of an image to correctly establish a threshold and perform an accurate analysis. Care has to be taken with this analysis because a small contrast variation across the MCP detector can lead to errors in threshold estimation that eventually translate into faulty numbers from the image analysis.

To analyze and understand the growth of TMA domains on $\mathrm{Cu}(001)$, our LEEM images are converted into binary form with the TMA domains appearing dark and the $\mathrm{Cu}(001)$ background appearing bright. The TMA domains grown at low temperature were small in area and, as a consequence, also had low contrast between the domains and $\mathrm{Cu}(001)$ surface, which prevented us from doing any detailed form of analysis. The analysis of larger domains formed during the room temperature growth experiments did turn out to be possible. The time dependence of the TMA domain density is shown in Fig. 3.8(a). In the initial stages of deposition, contrast between the TMA domains and the background is small making it difficult to accurately analyze the data. Therefore, data is plotted only after $40 \mathrm{~s}$ of deposition when the contrast between the domains and background is sufficient to allow for an accurate analysis. The curve is divided into three regions. The first region shows a steep increase in domain density which is typical during the early stages of a nucleation and growth experiment [32]. Region II shows saturation where the domain density reaches its peak value and slowly decreases due to coalescence. In this stage, the TMA domains exclusively grow and start to coalesce. In region III, the rate of coalescence increases dramatically and the domain density decreases as more TMA is deposited.

TMA domains did not show mobility during any stage of the experiments. The 
high nucleation density and the absence of any shape fluctuations or diffusion of the domains hints at a low mobility of the individual TMA molecules on $\mathrm{Cu}(001)$. Annealing of the surface after deposition to generate more moblity only resulted in the decay of TMA domains. Even during decay, the position of the TMA domains remained fixed. The increased surface temperature sufficiently disturbs the fine balance between the surface-molecule and molecule-molecule interaction breaking the intermolecular bonds and leading to domain decay.

Even though the elongated shape of the domains that is observed in the later stages of the room temperature growth experiments hints at the presence of a long-range stabilizing interaction, we could not extend our TMA experiments/studies further, because of the low mobility of the TMA domains and their small size, which approaches the resolution limits of LEEM. If a low mobility of the TMA molecules is the reason behind the formation of small domains then the deposition and growth of TMA at a higher substrate temperature should help to form larger domains. A comparison between the low and room temperature measurements underlines the validity of the above argument for our case. The domains grown at room temperature in Fig. 3.7 are larger in size than those grown at low temperature, see Fig. 3.6. Therefore, it is logical to assume that the growth of TMA domains at even higher temperature will lead to the formation of domains of an even larger size. However, the prohibiting factor turns out to be the intermolecular interaction which is weak and rather sensitive to even a minute change in surface temperature. Another approach, which we eventually ended up pursuing, and which is described in Chapter 4, is to use larger organic molecules which can form larger domains.

To summarize this section, we can state that the TMA/Cu(001) system shows us that LEEM is indeed the most appropriate instrument for the kind of investigations we want to undertake. However, after observing the area of TMA domains, grown at low temperature and at room temperature, it is evident that a careful choice of the molecule that is used in the experiments is required to form domains of an observable size. Larger domains provide more opportunities to analyze the growth in the framework of competing interactions, e.g. by investigating domain boundary fluctuations. Moreover, the formation of molecular domains on the $\mathrm{Cu}(001)$ surface leads to a significant change of the work function. The resulting fields that exist at the boundaries of the molecular domains will make any kind of quantitative analysis impossible if the size of the domains is small with respect to the distorting effects.

\subsection{Organic thin films and radiation damage}

A second effect that was highlighted in our initial experiments, but was not yet discussed, and that could provide a potential stumbling block for further investigation is the occurrence of radiation damage to the molecules and domains during exposure to the electron beam. Electron microscopy, in principle, can have damaging effects on the sample that is being imaged. Depending on the energy and interaction, elastic or inelastic, electron beams can damage both inorganic and organic samples. Organic materials with both covalent and other, much weaker, bonds are in fact a prime candidate for e-beam induced degradation. 

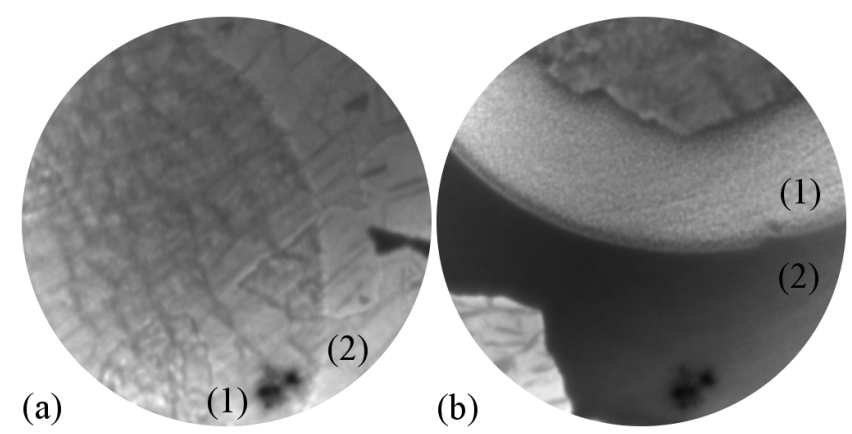

Figure 3.9:

LEEM images obtained from different surface locations at FoV $10 \mu \mathrm{m}$ with $1.5 \mathrm{eV}$ electron energy and an exposure of $3450 \mathrm{~s}$ to the electron beam. (a) BDA film covered graphene. Exposed (1) and unexposed (2) surface portion. (b) Graphene flakes are visible in the top and bottom part of the image. Exposed (1) and unexposed (2) surface portion to electron beam.

In literature, most studies of electron beam induced damage on aromatic materials are high energy transmission electron microscopy (TEM) and scanning electron microscopy (SEM) studies [33]. However, from these examples, we can learn about the interaction between electrons and organic matter and extrapolate the damage down to energies relevant to our experiments. Inelastic scattering causes sample heating. A stationary beam is more damaging then a scanning one. In the case of organic specimen, temperature can increase up to a few hundred degrees if the incident energy of a scanning beam is between $0.5 \mathrm{keV}$ and $2 \mathrm{keV}$ [33]. Aromatic compounds show more resistance to electron beam damage than other compounds. The presence of a stable ring structure with high resonance energy of the $\pi$-electrons is responsible for this stability where deposited energy is shared by many electrons without the breaking of any bonds [33].

Considering the electron energy between 1.5 and $3 \mathrm{eV}$ that is used in our LEEM experiments and the examples discussed above, we can safely assume that our organic samples should not be affected by the thermal effects mentioned above. However, low electron energies can also have damaging effects in the organic samples as shown in the Fig. 3.9. Therefore, a careful inspection of the sample is needed during and after the experiments even at low electron energies.

LEED, which is used concurrently with LEEM during this work, can have a more damaging effect on our samples because of the relatively high electron energy. Therefore, LEED measurements were always performed either in a very swift fashion that minimizes the total exposure to the electron beam or in areas that were totally separated from areas where LEEM images were recorded. This helped to eliminate completely or at least limit the damage that was observed.

To further illustrate the effect of the e-beam on the structure of the molecular domains in LEED measurements, we performed an experiment in which BDA domains were grown on the $\mathrm{Cu}(001)$ surface. LEED patterns were then measured, exposing the surface to the e-beam for a total time of $1800 \mathrm{~s}$. We found that the structure of the do- 

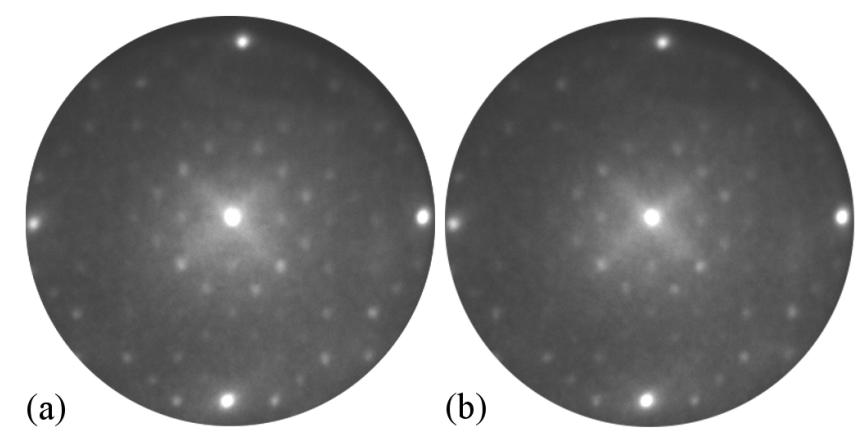

Figure 3.10:

LEED pattern obtained with electron energy of $29 \mathrm{eV}$ on a $\mathrm{Cu}(001)$ surface covered with $\mathrm{BDA}$ molecules. $(\mathbf{a}, \mathbf{t}=\mathbf{0} \mathbf{s})$ Start of LEED measurement. $(\mathbf{b}, \mathbf{t}=\mathbf{1 8 0 0} \mathrm{s})$ LEED pattern at the end measurement.

mains remained stable and the molecular arrangement was preserved, despite the high electron energy as shown in Fig. 3.10. The exposure time therefore appears to be the most important parameter that determines the amount of radiation damage in organic films. At low energies, a sample can be characterized for longer times compared to higher energies.

To summarize this section, we have observed radiation damage on organic thin films both at low (imaging mode of LEEM) and higher (LEED mode of LEEM) electron energies. To limit the radiation damage at high energies, LEED measurements must always be performed in a rapid fashion and equal care has to be taken to minimize the exposure of organic films to the beam during imaging at low energies. 


\section{Bibliography}

[1] The Concord Consortium (2011).

<http://www.mw. concord.org>

[2] G. M. Whitesides and B. Grzybowski, Science 295 (2002), 2418.

[3] R.M. Tromp and J.B. Hannon, Surf. Rev. Lett. 9 (2002), 1565.

[4] E.Y. Vedmedenko, Competing Interactions and Pattern Formation in Nanoworld, Wiley-Vch, Germany (2007), 74.

[5] J.B. Hannon, F.-J. Meyer zu Heringdorf, J. Tersoff, and R.M. Tromp, Phys. Rev. Lett. 86 (2001), 21.

[6] A.K. Schmid, K.L. Man, N.C. Bartelt, H. Poppa, and M.S. Altman, Microsc. Microanal. 9 (2003), 134.

[7] R. van Gastel, R. Plass, N.C. Bartelt, and G.L. Kellogg, Phys. Rev. Lett. 91 (2003), 055503.

[8] V.I. Marchenko and A.Ya. Parshin, Sov. Phys. JETP 52 (1980), 129.

[9] O.L. Alerhand, D. Vanderbilt, R.D. Meade, and J.D. Joannopoulos, Phys. Rev. Lett. 61 (1988), 1973.

[10] D. Vanderbilt, Surf. Sci. Lett. 268 (1992), L300.

[11] T. Garel and S. Doniach, Phys. Rev. B 26 (1982), 325.

[12] D.J. Keller, H.M. McConnell, and V.T. Moy, J. Chem. Phys. 90 (1986), 2311.

[13] D. Andelman, F. Brochard, and J.-F. Joanny, J. Chem. Phys. 86 (1987), 3673.

[14] H.M. McConnell, Annu. Rev. Phys. Chem. 42 (1991), 171.

[15] M. Seul and R. Wolfe, Phys. Rev. A 46 (1992), 7519.

[16] S.A. Langer, R.E. Goldstein, and D.P. Jackson, Phys. Rev. A 46 (1992), 4894.

[17] A.B. Kashuba and V. L. Pokrovsky, Phys. Rev. B 48 (1993), 10335.

[18] A.J. Dickstein, S. Erramilli, R.E. Goldstein, D.P. Jackson, and S.A. Langer, Science 261 (1993), 1012.

[19] M. Seul and D. Andelman, Science 267 (1995), 476.

[20] S.L. Keller and H.M. McConnell, Phys. Rev. Lett. 82 (1999), 1602.

[21] S.T. Nguyen, D.L. Gin, J.T. Hupp, and X. Zhang, Proc. Nat. Acad. Sci. 98 (2001), 11849.

[22] D. Payer, A. Comisso, A. Dmitriev, T. Strunskus, N. Lin, C. Wöll, A. DeVita, J.V. Barth, and K. Kern, Chem. Eur. J. 13 (2007), 3900. 
[23] A. Dmitriev, N. Lin, J. Weckesser, J.V. Barth, and K. Kern, J. Phys. Chem. B 106 (2002), 6907.

[24] S. Stepanow, M. Lingenfelder, A. Dmitriev, N. Lin, T. Strunskus, C. Wöll, J.V. Barth, and K. Kern, BESSY Highlights 2003 (2004), 20.

[25] G. Sheerin and A.A. Cafolla, Surf. Sci. 577 (2005), 211.

[26] S. Stepanow, M. Lingenfelder, A. Dmitriev, H. Spillmann, E. Delvigne, N. Lin, X. Deng, C. Cai, J.V. Barth, and K. Kern, Nat. Mater. 3 (2005), 229.

[27] V. De Renzi, R. Rousseau, D. Marchetto, R. Biagi, S. Scandolo, and U. del Pennino, Phys. Rev. Lett. 95 (2005), 46804.

[28] J.V. Barth, G. Costantini, and K. Kern, Nature 437 (2005), 671.

[29] S. Stepanow, N. Lin, D. Paye, U. Schlickum, F. Klappenberger, G. Zoppellaro, M. Ruben, H. Brune, J.V. Barth, and K. Kern, Angew. Chem. Int. Ed. 46 (2007), 710 .

[30] N. Zhu, T. Osada, and T. Komeda, Surf. Sci. 601 (2007), 1789.

[31] J.V. Barth, J. Weckesser, N. Lin, A. Dmitriev, and K. Kern, Appl. Phys. A 76 (2003), 645.

[32] J.A. Venables, G.D.T. Spiller, and M. Hanbucken, Rep. Prog. Phys. 47 (1984), 399.

[33] R.F. Egerton, P. Li, and M. Malac, Micron 35 (2004), 399. 


\section{Growth, structure, and thermal stability of BDA-domains on $\mathrm{Cu}(001)$}

This chapter describes the growth of BDA on $\mathrm{Cu}(001)$ which has been studied using LEEM and selective area $\mu L E E D$. The emergence of large islands and hydrogen bonding to perpendicularly oriented, adjacent molecules is confirmed. The two benzene rings of adsorbed BDA are twisted along the molecular axis. Unconventional growth of the domains, followed by a second nucleation stage, is observed at room temperature. This unanticipated feature is attributed to the accumulation of stress in the islands. Ostwald ripening in the films and the decay of BDA-domains at $448 \mathrm{~K}$ exhibits features that are consistent with diffusion limited behaviour*.

*Published in J. Chem. Phys. 135 (2011), 24706. 


\subsection{Introduction}

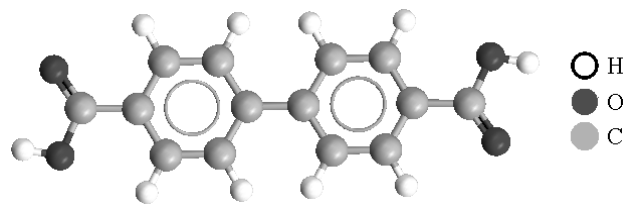

Figure 4.1:

The BDA molecule. BDA comprises of two phenyl rings and two identical carboxylic end groups.

The self-assembly of supra-molecular nanostructures is believed to be a viable step in the bottom-up route for deposition of functional molecular species on suitable substrates [1-6]. One of the more frequently studied building blocks is BDA on $\mathrm{Cu}(001)$ $[7,8]$. It has been reported that BDA molecules reside on $\mathrm{Cu}(001)$ as deprotonated dicarboxylic species [8-10]. BDA is an organic molecule with two phenyl rings and two functional carboxyl end groups. It is a non-chiral molecule, $1.3 \mathrm{~nm}$ in length and is shown in Fig. 4.1. BDA molecules self-assemble in a well-ordered, square twodimensional network structure on the $\mathrm{Cu}(001)$ surface at room temperature [7]. The molecules adsorb in a flat-lying geometry and form large domains when deposited on clean $\mathrm{Cu}(001)$ at room temperature.

Calculations show that the two benzene rings constituting the single BDA molecule are twisted along the long axis of the molecule [11]. Similar twisted benzene rings have been observed previously $[12,13]$. However, for BDA, both rings are expected to be in-plane when adsorbed on, e.g. Au(111) [11]. Adjacent molecules are rotated by $90^{\circ}$ with respect to each other. The lateral molecule-molecule interaction is governed by hydrogen bonding which is the driving force for the square ordering geometry.

As examplified in Fig. 2(a) of Ref. [7], the ordering is almost perfect. However, the hydrogen bonds are relatively weak, which results in a relatively low thermal and mechanical stability. The purpose of the work presented in this chapter is to shed light on the growth of the domains and their thermal stability. The present study is performed using LEEM [14] and selective area $\mu$ LEED.

\subsection{Experimental}

The substrate used in our experiments is an atomically clean and ultra-smooth [15] $\mathrm{Cu}(001)$ crystal mounted in ultrahigh vacuum. It was cleaned by 48 hours of annealing in an $\mathrm{H}_{2} / \mathrm{Ar}$ atmosphere, followed by repeated cycles of $\mathrm{Ar}^{+}$sputtering, and prolonged annealing at $900 \mathrm{~K}$. Commercially available BDA in powder form was deposited by OMBE from a Knudsen-cell type evaporator. In a first deposition experiment, the temperature of the evaporator was gradually ramped up from $413 \mathrm{~K}$ to $463 \mathrm{~K}$ to calibrate the deposition rate at which BDA domains are grown. A sublimation temperature of $463 \mathrm{~K}$ yielded a deposition rate of approximately one monolayer per hour. In subsequent experiments, similar deposition rates were used. The substrate temperature never exceeded $448 \mathrm{~K}$ during the experiments on BDA films to avoid thermal decomposition of the adsorbed molecules.

For imaging, primary energies in the range $2-2.5 \mathrm{eV}$ have been used. Under these 

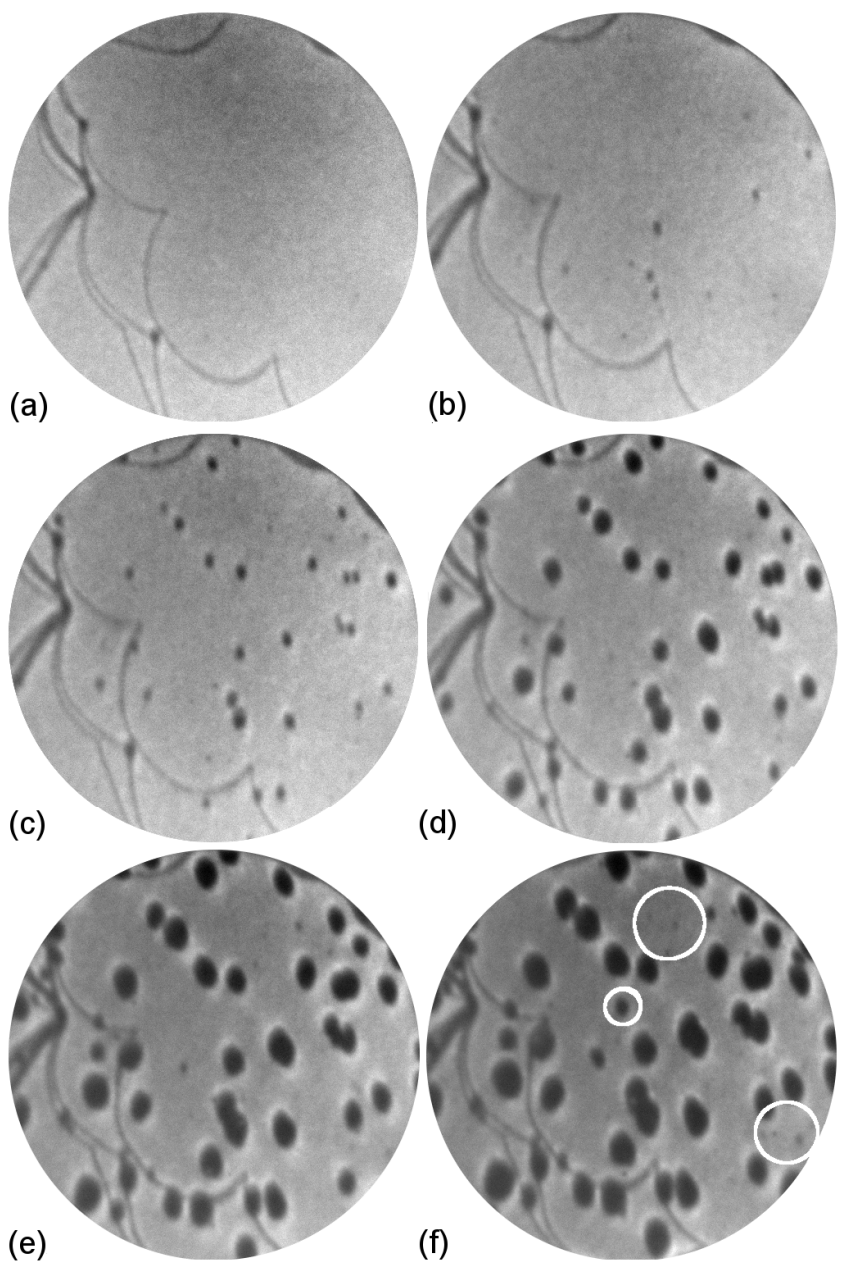

Figure 4.2:

Temporal evolution of $\mathrm{BDA}$ domains on $\mathrm{Cu}(001)$ at room temperature. The FoV is $3 \mu \mathrm{m}$ and the electron energy is $2.0 \mathrm{eV}$. The curved features represent steps and step-bunches. $(\mathbf{a}, \mathbf{t}=\mathbf{0} \mathbf{s})$ The clean $\mathrm{Cu}(001)$ surface at the start of the experiment. $(\mathbf{b}, \mathbf{t}=\mathbf{2 1 5 0} \mathbf{s})$ The start of domain nucleation. $(\mathbf{c}, \mathbf{t}=\mathbf{2 2 5 0} \mathrm{s})$ and $(\mathbf{d}, \mathbf{t}=\mathbf{2 5 5 0} \mathrm{s})$ Existing domains grow and nucleation of several new domains is observed. $(\mathbf{e}, \mathbf{t}=\mathbf{3 0 2 2} \mathbf{s})$ and $(\mathbf{f}, \mathbf{t}=\mathbf{3 3 1 2} \mathbf{s})$ The circles highlight sites at which unexpected late nucleation of new domains is observed. 


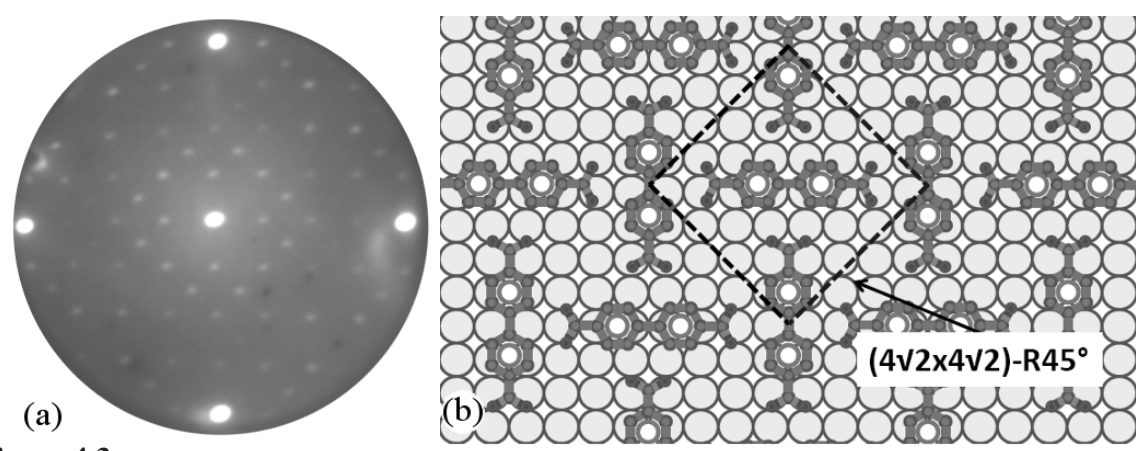

Figure 4.3:

(a) Typical LEED pattern of a Cu(001) surface covered with BDA domains taken at $29 \mathrm{eV}$. The bright features refer to the zero and first order $\mathrm{Cu}(001)$ spots. (b) Schematic of the arrangement of the BDA molecules in the domains on $\mathrm{Cu}(001)$.

circumstances, we have not detected any evidence for beam induced damage to the BDA domains during the prolonged experimental periods. The use of about $30 \mathrm{eV}$ electrons to obtain LEED patterns does lead to visible degradation of the BDA films. The timescale of the degradation, however, still allows for the acquisition of representative diffraction patterns. We judge the relative accuracy of the temperature to be $\pm 3 \mathrm{~K}$.

\subsection{Results}

\subsubsection{Growth at room temperature}

We have first deposited BDA with the $\mathrm{Cu}(001)$ substrate held at room temperature. Fig. 4.2 shows a sequence of representative LEEM images taken during growth. Fig. 4.2(a) shows the clean surface. The nucleation of BDA domains is observed only after a long initiation time of about 2100 seconds as witnessed by the appearance of dark areas in the Figs. 4.2(b) through (f). The BDA domains nucleate randomly, i.e., both (mostly) on the terraces and along the atomic steps as shown in Figs. 4.2(b) and (c). This indicates that heteronucleation on steps does not play a dominant role. A comparison of Figs. 4.2(b) and (c) shows the nucleation of many new domains. Figs. 4.2 (c) to (f) document the growth of existing domains while only a few new domains nucleate at a late stage (compare Fig. 4.2(e) and (f)). However, here nucleation takes place at a markedly lower rate than in earlier stages of the experiment.

We note that the growth rates of the domains that nucleated in the early phase of the experiment are quite similar whereas those of domains that nucleated in the later stages can differ substantially.

One distinct feature of the growth is the complete absence of mobility of BDA domains on the surface. An attempt to make them mobile by increasing the surface temperature resulted in the decay and disappearance of BDA domains (see further below). To elucidate the structure formed by the BDA on $\mathrm{Cu}(001)$, a representative $\mu$ LEED pattern taken from the $\mathrm{Cu}(001)$ surface covered with BDA domains is shown in Fig. 4.3(a). 
Figure 4.4:

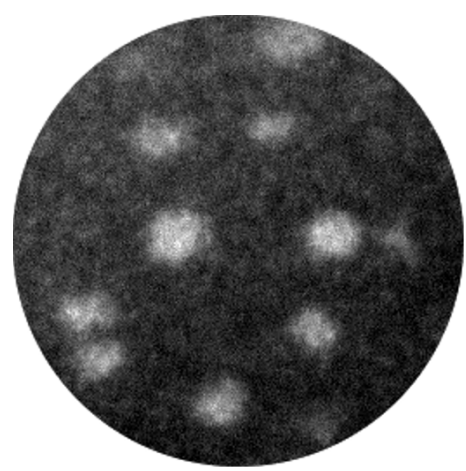

A dark field LEEM image taken by selecting one of the fractional order diffraction spots from the $B D A$ structure on $\mathrm{Cu}(001)$. The BDA domains yield a bright contrast compared to the $\mathrm{Cu}(001)$ surface. FoV is $5 \mu \mathrm{m}$ and electron energy is $2.5 \mathrm{eV}$.

The LEED pattern in Fig. 4.3(a) has been obtained with $29 \mathrm{eV}$. The bright spots refer to the $(0,0)$ spot and to the first order spots from $\mathrm{Cu}(001)$. The sharp superstructure spots reveal that the BDA domains are well ordered indeed, in agreement with Ref. [7]. It is noted that at this energy the quality of the LEED pattern degrades on a time scale of several minutes, indicative of electron beam induced damage in the domains at this higher energy. All domains have a well defined $(4 \sqrt{2} \times 4 \sqrt{2})$-R $45^{\circ}$ superstructure. This is also in line with the dark-field LEEM measurements using the superstructure spots, shown in Fig. 4.4. This illustrates that the intensity in the superstructure spots indeed originates from the BDA domains.

As mentioned in the introduction section of this chapter, the BDA molecules deprotonate on $\mathrm{Cu}(001)$ [8-10] and self-assemble into a well-ordered two-dimensional structure. They are oriented in a mutually perpendicular fashion with their carboxylic end groups hydrogen bonded to the benzene rings $[7,8]$ as shown in Fig. 4.3(b). The real space $(4 \sqrt{2} \times 4 \sqrt{2})-\mathrm{R} 45^{\circ}$ structure gives rise to the LEED pattern in Fig. 4.3(a).

It corresponds at first sight to a $c(8 \times 8)$ pattern with two glide lines along the diagonal directions ([110] and [-110]), due to two perpendicular pairs of flat lying molecules within the unit cell. However, a close inspection of the LEED patterns at various energies between 2 and $27 \mathrm{eV}$ (Ref. [16]) reveals that, depending on the primary energy, substantial intensity is carried by the symmetry forbidden peaks as for instance $(1 / 8,1 / 8),(1 / 8,7 / 8),(3 / 8,5 / 8),(5 / 8,3 / 8)$, and $(7 / 8,1 / 8)$. The corresponding lower symmetry is attributed to substantial twisting of the molecules along their axes, as elaborated in the supporting material [16]. This twisting is known from the gas phase too.

We have analyzed the data in Fig. 4.2 in more detail. A few observations are immediately evident. First, we note the long deposition time, i.e. relatively high coverage, prior to nucleation. At room temperature the onset of nucleation is only seen after about $2100 \mathrm{~s}$. The density of nuclei is quite low with about $1.7 \times 10^{-5} \mathrm{~nm}^{-2}$. As noted above, the system does not show any evidence for dominant heteronucleation behaviour. Furthermore, the complete absence of mobility of BDA domains is remarkable in view of the high mobility of the individual BDA molecules and the weak hydrogen type bond- 

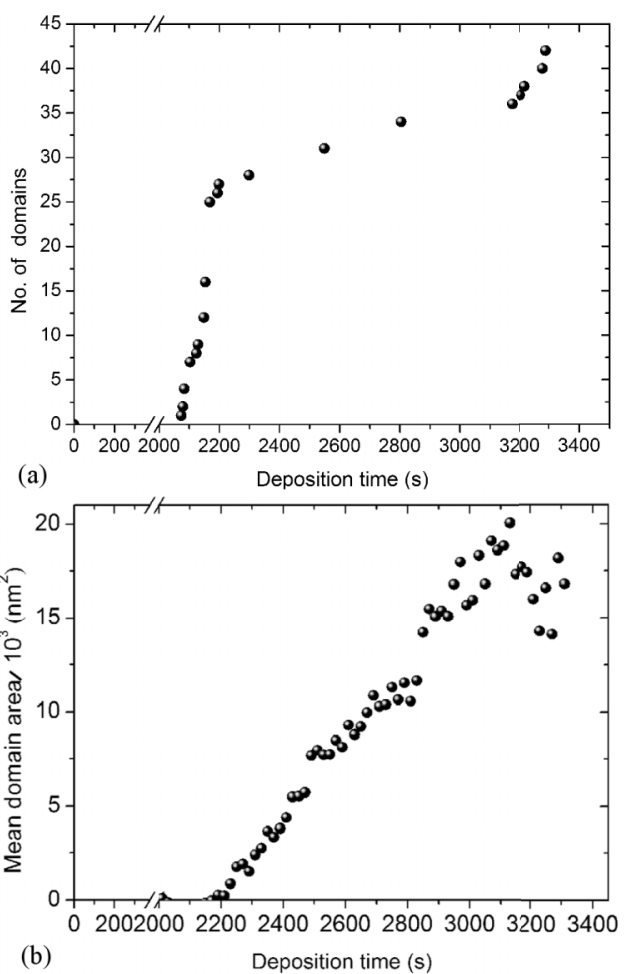

Figure 4.5:

(a) The number of domains as a function of deposition time within the FoV of $3 \mu m$ (cf. Fig. 4.2).

(b) The average area of the domains versus deposition time.

ing between the molecules in the domains. We focus first on the nucleation behaviour.

Fig. 4.5(a) shows the number of domains in an image with a FoV of $3 \mu \mathrm{m}$ for the experiment illustrated in Fig. 4.2. We can distinguish four regimes: i) 0-2100 s: the build-up of a sufficiently high BDA concentration to enable nucleation, ii) around $2100 \mathrm{~s}$ : nucleation commences, iii) $2150-3200 \mathrm{~s}$ : the nucleation phase is completed (only very few new nuclei appear) and the existing nuclei grow and expand and, most surprisingly, iv) after about $3200 \mathrm{~s}$, a second nucleation phase sets in. This behaviour is unconventional since one typically expects the curve to flatten out and even decay to lower values in later stages due to coalescence. Fig. 4.5(b) shows the accompanying average area of the measured domains versus deposition time. As expected before coalescence, the mean size of the islands grows linearly. It is stressed that the onset of the unconventional second late nucleation regime is also apparent here. It is intimately related to a maximum mean size of the island of roughly $1.7 \times 10^{4} \mathrm{~nm}^{2}$, or $8.2 \times 10^{3}$ BDA unit cells. Its linear size corresponds to 90 base vectors.

The emergence of a maximum size of the BDA islands is unexpected and needs to be discussed here. The binding energy of a benzene ring to a $\mathrm{Cu}(001)$ surface is calculated to be $0.68 \mathrm{eV}$ [17]. The entire molecule is rather tightly bound with approxi- 
mately $1.36 \mathrm{eV}$. The influence of the corrugated potential energy surface of the substrate on the orientation is quite strong as comprehensively illustrated by the extended square patterns of BDA on $\mathrm{Cu}(001)$ [7] and the nicely ordered LEED pattern in Fig. 4.2. A closer inspection of the cartoon in Fig. 4.3(b) reveals that the hydrogen bond length is about $0.225 \mathrm{~nm}$, i.e., well above, for instance, water $(0.197 \mathrm{~nm})$. So one may conclude that the BDA domains are under tensile stress due to their intimate connection with the substrate. Apparently, as the domains grow beyond a linear size of more than 90 base vectors, a critical value of the stress induced energy is passed. Thinking along the lines of the well-known Frenkel-Kontorova model, the next molecules that are added can no longer be accommodated in a potential minimum offered by the substrate. Taking the values for water as a guide, the thermal expansion coefficient of the molecular layer is about 10-40 times larger than that of copper. Therefore, the critical size could be strongly temperature dependent and would increase with temperature.

Together with the data in Fig 4.5(a), the linear rise in Fig. 4.5(b) provides an excellent possibility to calibrate the deposition rate. We arrive at a deposition rate of $1.0 \times 10^{-4}$ monolayers per second. This number implies that the spreading pressure corresponds to a molecular density of no less than $21.5 \%$ of the condensed layer. Taking into account that the monolayer corresponds to one molecule per 16 surface atoms, the spreading pressure corresponds to $1.35 \times 10^{-2} \mathrm{BDA}$ molecules per copper surface atom. Unfortunately, the island density in Fig. 4.2 is too low, i.e. the statistics is too poor to apply a rate equation approach [18] in order to make an estimate of the critical size for nucleation or to even determine the binding energy of the molecules in the domain.

\subsubsection{Continued growth of the BDA domains at $448 \mathrm{~K}$}

Slow heating of the substrate from room temperature to $448 \mathrm{~K}$ leads to a gradual decay of the BDA domains. This is attributed to a two-dimensional sublimation of the BDA molecules as a result of a subtle interplay between the intermolecular forces and their registry with the surface. The molecules become part of the two-dimensional diluted phase on the surface as will be discussed further below. At $448 \mathrm{~K}$, the BDA domains have disappeared completely as illustrated in Fig. 4.6(a). After stabilization of the temperature at $448 \mathrm{~K}$, we have reopened the shutter of the Knudsen cell. The starting point (new time origin), thus, corresponds to a concentration of $34.6 \%$ of a monolayer in the dilute phase or $0.022 \mathrm{BDA}$ molecules per copper surface atom. The surface morphology that evolves at a deposition rate of $1.0 \times 10^{-4}$ monolayers per second is shown in Fig. 4.6. Again it takes a prolonged time before resumed nucleation of domains commences. This occurs at a total BDA coverage of about $0.72 \mathrm{ML}$ corresponding to about 0.045 molecules per copper surface atom. Again the new domains are formed on the terraces. Fig. 4.7 shows the evolution of the number of nuclei and their average size during deposition. Nucleation occurs after 3715 seconds and the average size increases linearly. This time, no deviation from normal nucleation and growth behaviour is detected, i.e. no evidence is found for a maximum island size and the consequent second stage nucleation events. Considering the fact that the system is at a $155 \mathrm{~K}$ higher temperature, this is in line with the earlier explanation. If we again compare with the thermal expansion coefficients of water which are about 10-40 times 

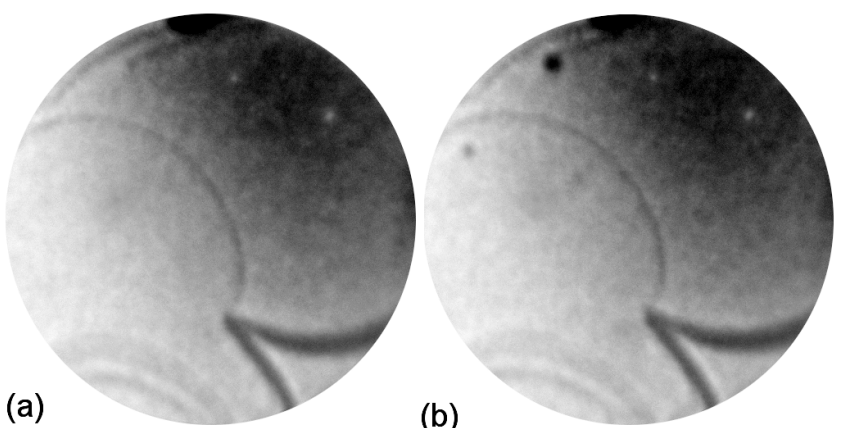

(a)

(b)
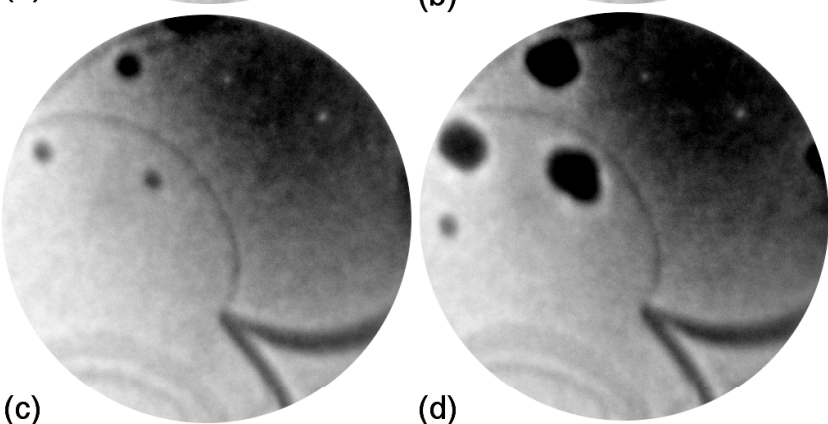

(c)

(d)
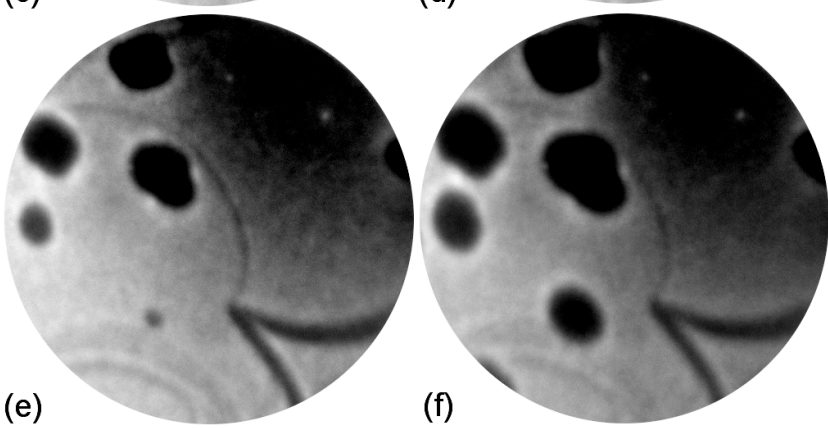

Figure 4.6:

BDA deposition and growth at $448 \mathrm{~K}$. Bright spots visible in the top right side of the images are defects in the detector. FoV is $3 \mu \mathrm{m}$ and electron energy is $2.5 \mathrm{eV}$. $(\mathbf{a}, \mathbf{t}=\mathbf{3 6 9 7} \mathrm{s})$ Single surface atomic steps and step bunches appear as curved features. $(\mathbf{b}, \mathbf{t}=\mathbf{3 7 2 0} \mathrm{s})$ Nucleation of three BDA domains. $(\mathbf{c}, \mathbf{t}=\mathbf{3 7 3 0} \mathrm{s}),(\mathbf{d}, \mathbf{t}=\mathbf{3 7 9 8} \mathrm{s}),(\mathbf{e}, \mathbf{t}=\mathbf{3 7 6 5} \mathrm{s})$, and $(\mathbf{f}, \mathbf{t}=\mathbf{3 8 3 7} \mathrm{s})$ The growth of domains and the nucleation of some new domains. The integral coverage in terms of monolayers is 0.716 , $0.718,0.719,0.723,0.726$, and $0.730 \mathrm{ML}$, respectively. 

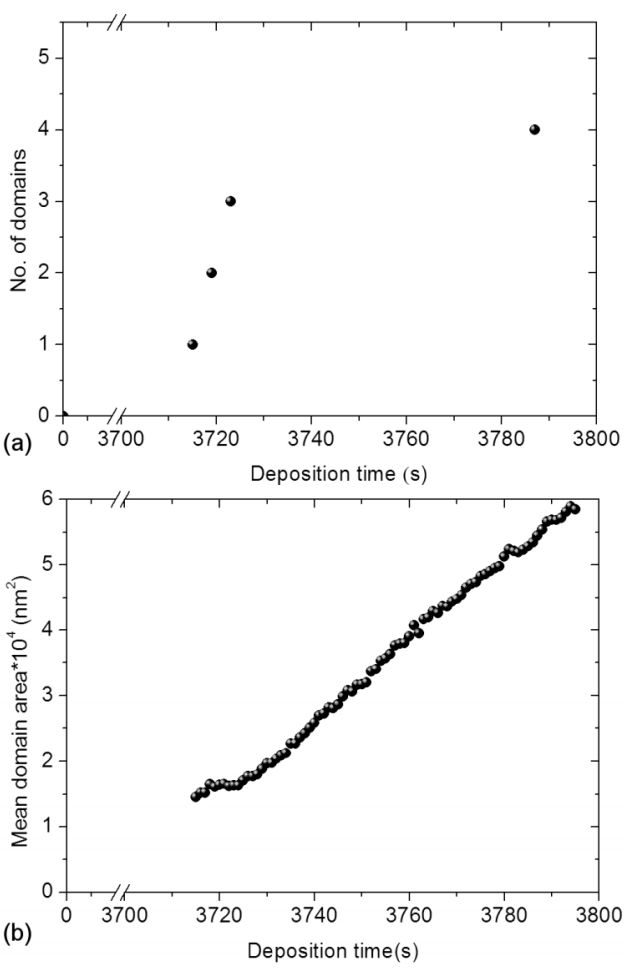

Figure 4.7:

The data is taken from the images shown in Fig. 4.6. (a) The number of domains versus deposition time with the substrate at $448 \mathrm{~K}$. (b) The mean domain size versus deposition time.

larger than that for copper, the hydrogen bond length at $448 \mathrm{~K}$ may be increased by several percents compared to that at $293 \mathrm{~K}$. As a result, the tensile stress will be much lower and there is no longer an apparent reason for a restricted size of the domains.

However, Fig. 4.7(b) illustrates another unexpected observation. From the slope of Fig. 4.7(b), we conclude that the integrated growth rate of the domains is about $2 \times 10^{-3}$ monolayers per second. This value is a remarkably factor of $\sim 20$ higher than the one obtained at room temperature, with identical settings of the Knudsen cell. This can be rationalized by the fact that nucleation involves a Gibbs free energy barrier which is constituted by the interplay between the cost term for the interface (domain boundary) and the gain term determined by the supersaturation, $\Delta \mu$, for molecules within the domains. For two-dimensional condensation, the interface term is proportional to the boundary length of the nucleus and the areal term corresponds to the size of the nucleus. The latter is proportional to the coverage, $\theta$, while the former scales with $\sqrt{\theta}$. Obviously, the latter always prevails at high coverage in the case of supersaturation. Note that we deposit material at a fixed rate. Therefore, the supersaturation is much higher at room temperature than at $448 \mathrm{~K}$. Since the Gibbs free energy barrier for twodimensional nucleation is proportional to $1 / \Delta \mu$ [19], it is much higher at $448 \mathrm{~K}$. The higher growth rate of the BDA domains at $448 \mathrm{~K}$ can now be explained by a substantial 
contribution from the two-dimensional diluted phase after nucleation. After $3795 \mathrm{~s}$, the integrated coverage of the domains is $0.15 \mathrm{ML}$. At that point, the density of the diluted phase is still equivalent to $0.68 \mathrm{ML}$ or 0.042 molecules per copper surface atom. Eventually, the growth rate should level off. Unfortunately, the nucleation stage has not yet been concluded (cf. Fig. 4.7(a)). So we cannot determine the corresponding spreading pressure [20] at $448 \mathrm{~K}$. However, one can take the value of 0.042 molecules per copper atom as an overestimate. With a value of 0.014 molecules per copper atom at room temperature, we obtain an upper estimate of the two-dimensional cohesive energy for $\mathrm{BDA}$ on $\mathrm{Cu}(001)$ of about $0.08 \mathrm{eV}$ per molecule. This value is in the range expected for weak hydrogen bonds.

As mentioned above, a comparison of Figs. 4.5 and 4.7 shows that the integrated expansion rate of the domains differs by a factor of $\sim 20$, being higher at $448 \mathrm{~K}$ than at room temperature. Finally, the question may then be raised how accurate the deposition rate, as determined from Fig. 4.5(b), really is? To answer this question, it is reminded that the high expansion rate of the domains, as shown in Fig. 4.7 (b), is attributed to post-nucleation contributions from the two-dimensional diluted phase as a consequence of a high free energy barrier for nucleation at $448 \mathrm{~K}$. Since the supersaturation is much higher at room temperature, and consequently the free energy barrier for nucleation is much lower, we estimate that the previously derived deposition rate is accurate.

\subsubsection{Thermal stability of the BDA domains}

Closing the shutter after prolonged deposition at $448 \mathrm{~K}$ as described above leads to a gradual disappearance of the BDA domains, i.e. the islands sublimate in twodimensions: the molecules form a diluted or two-dimensional gas phase. This process can be suppressed by lowering the substrate temperature as demonstrated in Fig. 4.8. Fig. 4.8 initially shows three BDA domains present on different terraces (panel (a)). Increasing the temperature leads to a decay of these islands (panel (b)) and finally their complete disappearance (panel (c)) as a result of two-dimensional sublimation. This is corroborated further by the fact that the islands reappear upon lowering the surface temperature (panels (d)-(f)). This temperature cycle can be repeated as often as desired. A comparison of panels (a) and (f), both recorded at $296 \mathrm{~K}$, reveals that both the lateral distribution and the size distribution of the islands depends on the thermal history (temperature trajectory and rate of change). It is also evident that the islands nucleate at different sites supporting the argument for homogeneous nucleation.

\subsubsection{Ostwald ripening}

It is well known that for circular two-dimensional structures with a finite radius of curvature, $r$, the equilibrium density of diffusing particles in the immediate vicinity of the island, $\rho_{r}$, differs from the equilibrium density for infinitely large islands, $\rho_{\infty}$, and is given by the Gibbs-Thomson relation:

$$
\rho_{r}=\rho_{\infty} \exp [\gamma \Omega / k T r]
$$

with $\gamma$ the line tension of the edges and $\Omega$ the area per molecule.

$$
\Delta \rho=\left(\rho_{r}-\rho_{\infty}\right)
$$



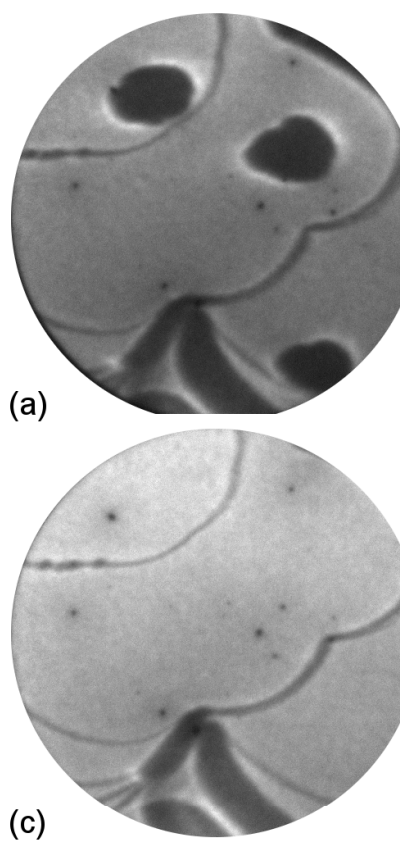

(b)
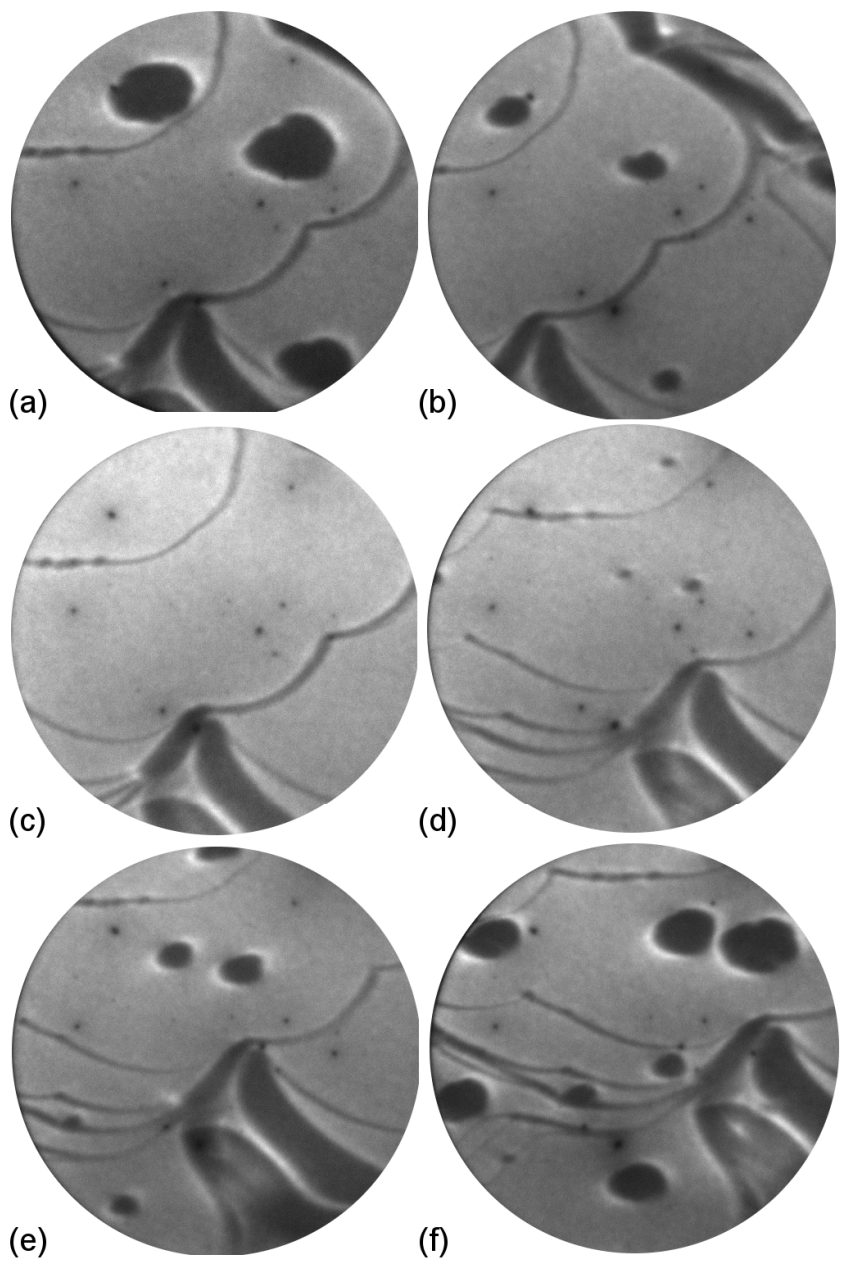

\section{Figure 4.8:}

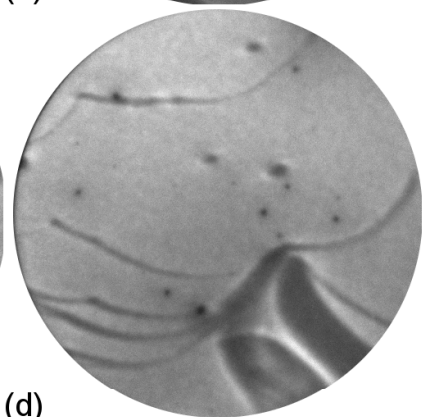

$3 \mu \mathrm{m}$ FoV LEEM images acquired at an electron energy of $2 \mathrm{eV}$. $(\mathbf{a}, \mathbf{T}=296 \mathbf{K})$ The BDA domains were grown by the deposition of BDA molecules as described in the text. $(\mathbf{b}, \mathbf{T}=\mathbf{4 1 7} \mathbf{K})$ The domains decay with increasing surface temperature. $(\mathbf{c}, \mathbf{T}=\mathbf{4 2 8} \mathbf{K})$ All BDA domains have completely vanished (the small black spots are due to damage in the channel-plate). $(\mathbf{d}, \mathbf{T}=\mathbf{3 2 0} \mathbf{K})$ The BDA domains reappear upon lowering the surface temperature. $(\mathbf{e}, \mathbf{T}=\mathbf{3 1 4} \mathbf{K})$ and $(\mathbf{f}$, $\mathbf{T}=\mathbf{2 9 6} \mathbf{K})$ The BDA domains grow in size with decreasing surface temperature. 


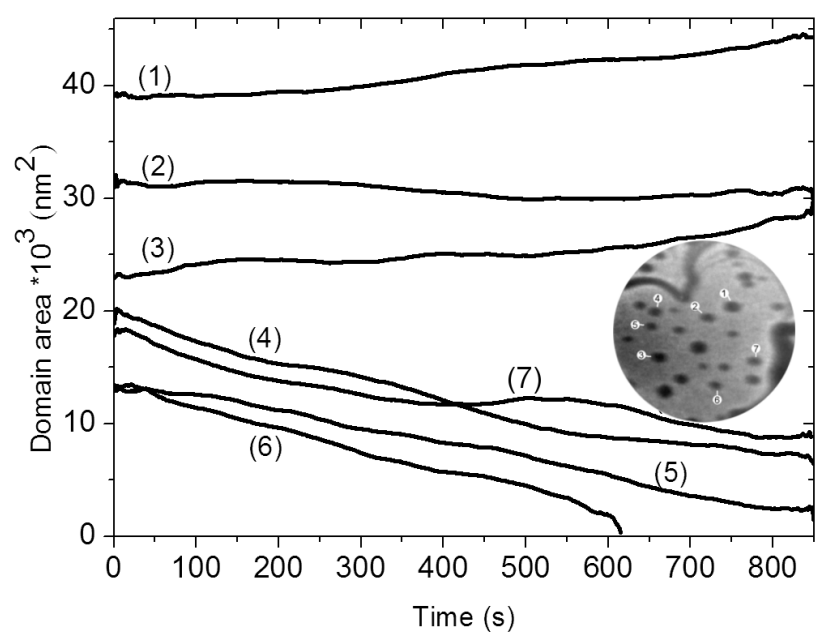

Figure 4.9:

The temporal evolution of a few domains at $373 \mathrm{~K}$, marked in the insert with a FoV of $3 \mu \mathrm{m}$ and electron energy of $2.5 \mathrm{eV}$.

is the driving force for growth or decay. If the presence of neighbouring islands is neglected, the asymptotic decay of the area of an island is given by:

$$
\Sigma=C\left(t_{0}-t\right)^{\alpha},
$$

where $C$ is a constant. The time exponent $\alpha$ depends on the physics and equals, for instance, 1 for interface limited decay and 2/3 for diffusion limited island decay [2123]. In the diffusion limited case, the rate of mass transport is determined by the gradients in the particle density on the terraces [24, 25]. Diffusion limited decay (or ripening), thus, leads to a significant influence of the island positions on the rate of change of the island area. For example, two-dimensional Ag-islands on $\mathrm{Ag}(111)$ decay with different rates despite having a similar size.

Ostwald ripening also occurs for the $\mathrm{BDA} / \mathrm{Cu}(001)$ system at $373 \mathrm{~K}$ as illustrated in Fig. 4.9. Substantial changes in island areas take place on a time scale of hundreds of seconds. The results clearly demonstrate substantial ripening effects and diffusion limited behaviour. The general observation is that the larger islands survive at the cost of smaller ones. Islands of similar size may show growth or decay depending on their local environment. Even a cross-over between growth and decay is observed in a few instances.

\subsubsection{Temporal evolution of BDA domains at $448 \mathrm{~K}$}

We will now attempt to determine the characteristics of the island decay at a more elevated temperature of $448 \mathrm{~K}$. After the growth of domains at $448 \mathrm{~K}$, the deposition of BDA is stopped. Fig. 4.10 shows the temporal evolution of the area, $\Sigma$, of domain A (see insert). Its location on the large terrace defines a relatively isolated, well-defined configuration, i.e. no other domains are in its immediate vicinity and steps do not act as 


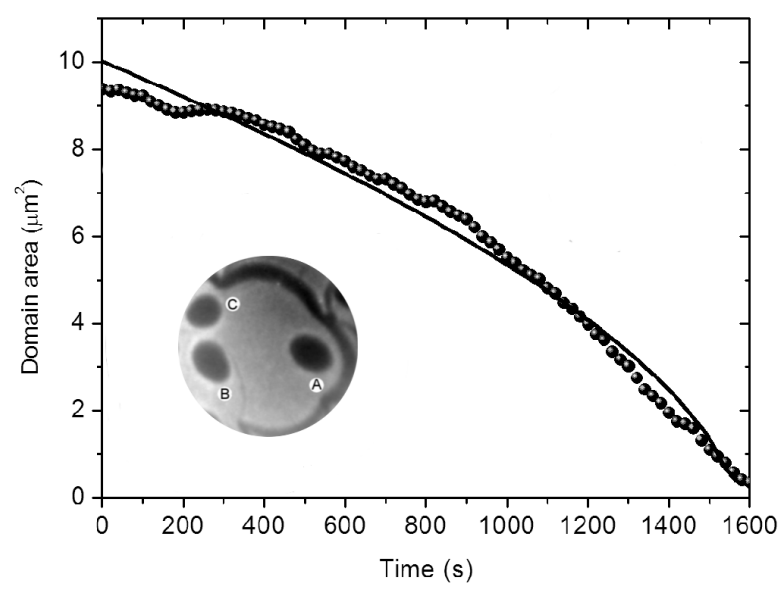

Figure 4.10:

The decay of domain A (see insert, FoV is $3 \mu \mathrm{m}$ and electron energy is $2.5 \mathrm{eV}$ ). The circles show the experimental data and the solid curve is a fit of the domain area as a function of time to: $\Sigma=C\left(t_{0}-t\right)^{\alpha}$. The resulting time exponent $\alpha=0.61 \pm 0.05$.

sinks for adsorbed BDA. The best fit to Eq. 4.3 is obtained for $\alpha=0.61 \pm 0.05$. This behavior reveals that diffusion limited decay still applies at this temperature for the decay of BDA domains on $\mathrm{Cu}(001)$. Similar behavior has been observed for perylenetetracarboxylic acid-dianhydride (PTCDA) on $\mathrm{Ag}(001)$ [25].

\subsection{Conclusions}

Using LEEM and $\mu$ LEED, we have studied the growth and stability of BDA domains on $\mathrm{Cu}(001)$. Preliminary results confirm the formation of large islands with a square arrangement of the individual BDA molecules. Adjacent molecules are oriented in a perpendicular fashion and their interaction is consistent with hydrogen stabilized bonds. $\mu$ LEED reveals that the benzene rings are twisted along the intramolecular axis. Growth at room temperature shows unconventional nucleation behavior: Nucleation occurs late and the subsequent growth of BDA-domains is interrupted when the islands reach a linear size of about 90 units. This feature is attributed to the accumulation of (tensile) stress which is no longer the case at $448 \mathrm{~K}$. Classic Ostwald ripening is observed in island ensembles at $373 \mathrm{~K}$. The decay of a large island at $448 \mathrm{~K}$ in a welldefined geometry shows scaling behavior with a time exponent $\alpha=0.61 \pm 0.05$. This is indicative of diffusion limited decay, in agreement with the observed Ostwald ripening features. 


\section{Bibliography}

[1] J.M. Lehn, Supramolecular Chemistry: Concepts and Perspectives, VCH, Weinheim, (1995).

[2] G.M. Whitesides, J.P. Mathias, and C.T. Seto, Science 254 (1991), 1312.

[3] D. Philip and J.F. Stoddart, Angew. Chem. Int. Ed. 35 (1996), 1154.

[4] J.K. Gimzewski and C. Joachim, Science 283 (1999), 1683.

[5] T.Yokoyama, S. Yokoyama, T. Kamikado, Y. Okuno, and S. Mashiko, Nature 413 (2001), 619.

[6] J.V. Barth, J. Weckesser, C. Cai, P. Gunter, L. Burgi, O. Jeandupeux, and K. Kern, Angew. Chem. 112 (2000), 1285.

[7] S. Stepanow, N. Lin, F. Vidal, A. Landa, M Ruben, J.V. Barth, and K. Kern, Nano lett. 5 (2005), 901.

[8] N. Lin, A. Dmitriev, J. Weckesser, J.V. Barth, and K. Kern, Angew. Chem. Int. Ed. 41 (2002), 4779.

[9] C.C. Perry, S. Haq, B.G. Frederick, and N.V. Richardson, Surf. Sci. 409 (1998), 512.

[10] S. Stepanow, T. Strunskus, M. Lingenfelder, A. Dmitiriev, H. Spillmann, N. Lin, J.V. Barth, C. Wöll, and K. Kern, J. Phys. Chem. B 108 (2004), 19392.

[11] N. Zhu, T. Osada and T. Komeda, Surf. Sci. 601 (2007), 1789.

[12] G.E. Poirier, Chem. Rev. 97 (1997), L254.

[13] J.V. Barth, G. Costantini, and K. Kern, Nature 437 (2005), 671.

[14] E. Bauer, Rep. Prog. Phys. 57 (1994), 895.

[15] U. Linke and B. Poelsema, J. Physics E: Sci. Instr. 18 (1985), 26.

[16] See supplementary material at <http://dx.doi.org/10.1063/1. $3641893>$ for additional background corrected LEED patterns and a discussion of the consequences of their symmetry for the orientation of the adsorbed BDA molecules.

[17] N. Lorente, M.F.G. Hedouin, R.E. Palmer, and M. Persson, Phys. Rev. B 68 (2003), 155401.

[18] J.A. Venables, G.D.T. Spiller, and M. Hanbücken, Rep. Prog. Phys. 47 (1984), 399.

[19] I.V. Markov, Crystal growth for beginners, World Scientific, Singapore, (2003).

[20] I.M. Lifshitz and V.V. Slyozov, J. Phys. Chem. Sold. 19 (1961), 35. 
[21] P.A. Thiel, M. Shen, D.-J. Liu, and J.W. Evans, J. Phys. Chem. C 113 (2009), 5047.

[22] M. Giesen, Prog. Surf. Sci. 68 (2001), 1.

[23] K.Morgenstern, Phys. Stat. Sol. B 242 (2005), 773

[24] G. Rosenfeld, K. Morgenstern, I. Stensgaard, W. Wulfhekel, E. Lægsgaard, F. Besenbacher, and G. Comsa, Surf. Sci. 402 (1998), 401.

[25] J. Ikonomov, C.H. Schmitz, and M. Sokolowski, Phys. Rev. B 81 (2010), 195428. 


\section{The role of topographical defects in growth of BDA film on graphene}

Following our LEEM study of BDA molecules on $\mathrm{Cu}(001)$, we have investigated the growth of the same molecule on the technologically relevant graphene surface, supported by an Ir(111) substrate. LEEM images provide direct insight into the growth dynamics and show that defects in the graphene play a crucial role in the final morphology of the molecular film that forms. BDA is demonstrated to form hydrogen bondstabilized chains on graphene. Dark-field LEEM images revealed that the same defects, which determine the morphology of the film, also direct the orientation of the domains, highlighting the importance of understanding the role of defects in epitaxial processes of organic molecules on graphene*.

*Published in Phys. Rev. B 82 (2010), 205409. 


\subsection{Introduction}

Graphene is a two-dimensional hexagonal lattice of carbon atoms and is attracting significant attention because of its unique electronic properties [1] and potential for applications in fields like nanoelectronics. To realize the true potential of graphene, it has to be grown in a controlled and reproducible manner that yields high quality graphene sheets. Subsequently, the graphene sheets need to be functionalized. Recently, substantial progress has been made in understanding and manipulating the growth of graphene sheets on $\mathrm{SiC}$ [2] and on metal surfaces like Ir [3-5], Ru [6, 7], and Ni [8]. Techniques for patterning and functionalizing the high quality graphene sheets that are now available must be developed. Some recent progress has been made [9], however, this field still requires detailed investigations to improve our understanding of the functionaliziation of graphene sheets using organic molecules for the application of graphene as, e.g. a transparent electrode material. In the work that is described in this chapter, we have investigated the adsorption, growth, and structure of BDA molecules on epitaxially grown graphene sheets [3-5] supported by an $\operatorname{Ir}(111)$ surface.

The BDA molecule is depicted graphically in Fig. 4.1. BDA is an organic molecule comprising two phenyl rings and two dicarboxylic acid functional end groups. It is a non-chiral molecule and $1.3 \mathrm{~nm}$ in length. Theory predicts a dihedral angle of $32^{\circ}$ between the two phenyl rings in the gas phase [10]. BDA has a well-established capability to form extended self-assembled, hydrogen bond stablized, two-dimensional networks [11-15]. It is also used to form two-dimensional host-guest systems [16, 17]. The patterns that were formed by the molecules and the influence of topographical defects that are present in the graphene sheets were imaged in real-time using LEEM [18], PEEM, and structurally characterized using $\mu$ LEED.

\subsection{Experimental}

The experiments were carried out in an Elmitec LEEM III with a base pressure less than $1 \times 10^{-10}$ mbar. An $\operatorname{Ir}(111)$ surface was atomically cleaned by exposing to low pressures of $\mathrm{O}_{2}$ at elevated temperature. Graphene films were then prepared by CVD of $\mathrm{C}_{2} \mathrm{H}_{4}$ on the $\operatorname{Ir}(111)$ surface at a temperature of $875 \mathrm{~K}$ [4]. All experiments were performed on graphene sheets that were orientationally aligned with the $\operatorname{Ir}(111)$ substrate [5, 19], unless otherwise mentioned. The growth of graphene sheets was followed in real-time using PEEM until sufficiently large graphene flakes had formed on the surface as shown in Fig. 5.1(a). All experiments were performed with the Ir surface partially covered by graphene. Commercially available BDA molecules in powder form were deposited by OMBE from a Knudsen-cell type evaporator that was held at a temperature of $523 \mathrm{~K}$.

\subsection{Results}

A LEEM image of a graphene flake on the $\operatorname{Ir}(111)$ surface and the consequent growth of molecular domains as the graphene is exposed to BDA, is shown in Fig. 5.1. $\operatorname{Ir}(111)$ surface steps are visible as thin lines in Fig. 5.1(a) as the graphene is forced to follow the contours of the underlying substrate. A network of straight linear features, appearing much darker than the steps, is also visible on the graphene. These linear 


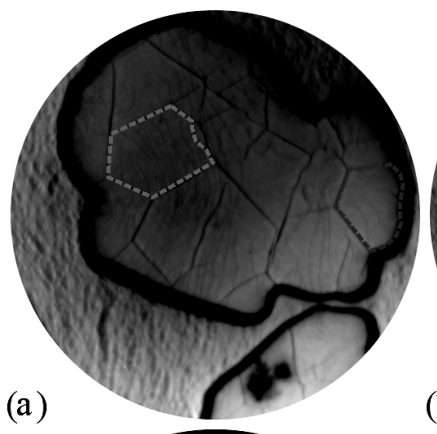

(a)
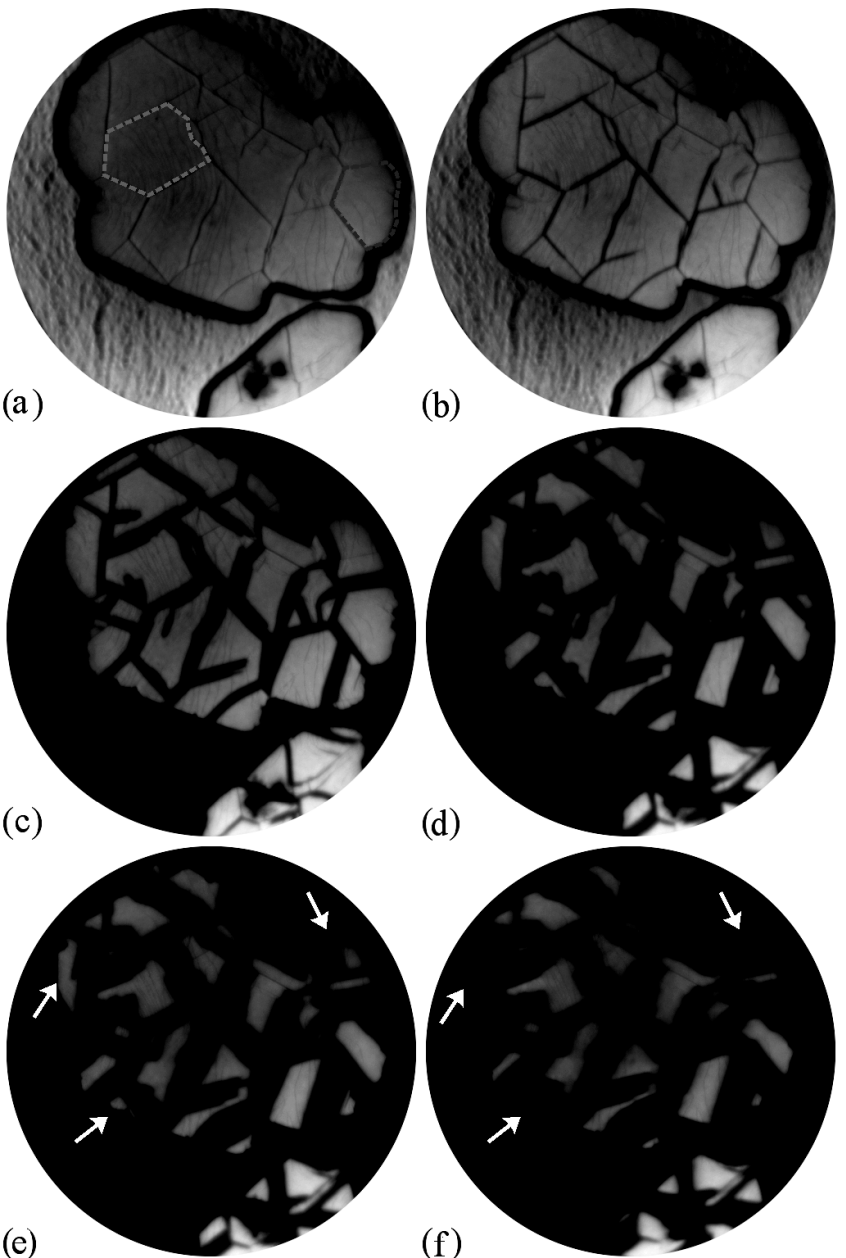

Figure 5.1:

(f)

$(\mathbf{a}, \mathbf{t}=\mathbf{0} \mathbf{s}) 10 \mu \mathrm{m}$ FoV bright-field LEEM image acquired at an electron energy of $1.5 \mathrm{eV}$ and temperature of $347 \mathrm{~K}$. Times indicated for the subsequent panels are measured with respect to the start of BDA deposition. Two graphene flakes are shown on the Ir(111) surface prior to exposure to BDA. Wrinkles and the contours of the underlying Ir(111) substrate steps are visible on the monolayer thick graphene flakes. The dark appearance of the edges of the graphene flakes is a consequence of a potential difference between the graphene and the Ir(111) that locally distorts the LEEM image. The areas used for analysis are indicated by the red and blue dashed lines. $(\mathbf{b}, \mathbf{t}=\mathbf{4 0} \mathbf{s})$ Domains of BDA molecules form and are exclusively observed to decorate wrinkles in the graphene sheet. $(\mathbf{c}, \mathbf{t}=\mathbf{1 9 0} \mathrm{s})$ Growth of domains occurs through lateral expansion of the domains perpendicular to the wrinkles. $(\mathbf{d}, \mathbf{t}=\mathbf{3 0 4} \mathbf{s})$ The shutter of the evaporator is now closed. $(\mathbf{e}, \mathbf{t}=\mathbf{1 8 5 0} \mathbf{s})$ Post-deposition growth of BDA domains on the graphene that are directly adjacent to the bare $\operatorname{Ir}(111)$ substrate is observed as indicated by the arrows. $(\mathbf{f}, \mathbf{t}=\mathbf{4 0 0 0} \mathbf{s})$ Edge regions of the graphene flake are now almost fully covered by the BDA domains. 

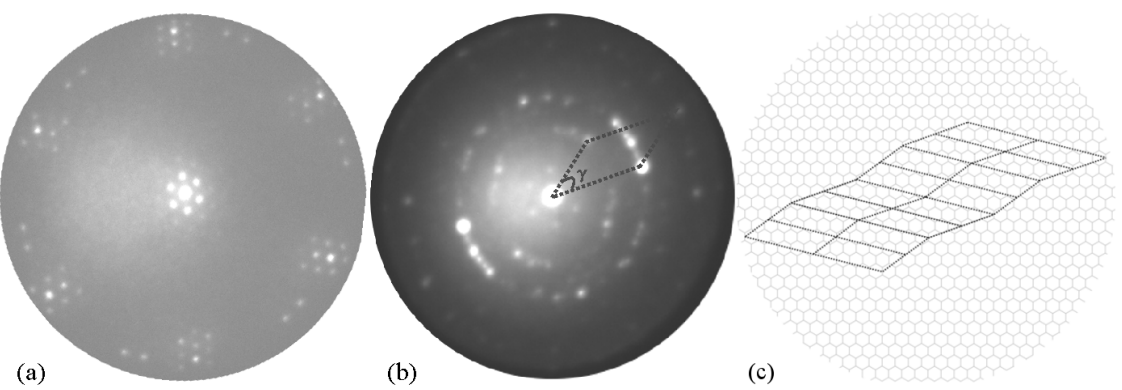

Figure 5.2:

(a) A $\mu L E E D$ pattern of a clean graphene surface taken with an electron energy of $42.4 \mathrm{eV}$ and a 1.4 um field-limiting aperture. The LEED pattern shows a moiré of graphene domains that are aligned with respect to the substrate [19]. (b) A $\mu L E E D$ pattern of BDA covered graphene taken with an electron energy of $14.7 \mathrm{eV}$ and a $1.4 \mu \mathrm{m}$ field-limiting aperture. The specular reflection and other LEED spots associated with various rotational domains of the ordered BDA structure are visible. The image is magnified with respect to panel (a). (c) The structural model proposed from the $\mu L E E D$ pattern shown in (b). The BDA molecules and the chains are positioned in the diagonal directions of the unit cells and hydrogen bonds set on the bridge sites. The aromatic rings are tilted with respect to the surface.

features are wrinkles in the graphene sheet that result from elastic relaxations that occur when the sample is cooled from the graphene growth temperature to $347 \mathrm{~K}$ for the BDA deposition $[19,20]$. The wrinkles vertically extend about $3 \mathrm{~nm}$ from the surface and are a few nanometers in width. They have been observed not just in graphene on $\operatorname{Ir}(111)[19,20]$, but also on exfoliated graphene [21], $\mathrm{SiC}$ [22], and other metals like $\mathrm{Ni}$ [23, 24], Pt [25], and $\mathrm{Cu}$ [26]. Fig. 5.1(b) illustrates the inhomogeneous nucleation of molecular domains as BDA is deposited. The domains decorate the wrinkles indicating that the BDA molecules preferentially reside near graphene wrinkles after adsorption from the vapor phase. No nucleation of domains was observed on the terraces between the wrinkles or on the edges of graphene flakes. After nucleating, the domains grow in a very distinct fashion. First, they extend parallel to the wrinkles to form linear domains until the wrinkles are fully covered. The growth of a domain along a wrinkle ceases as it impinges on other domains and wrinkles. This is followed by a widening of the domains perpendicular to the wrinkles, shown in Fig. 5.1(c). Nucleation of a second layer is not observed. A final, striking observation is made after the shutter of the BDA evaporator is closed in Fig. 5.1(d). Those regions that are directly adjacent to the $\operatorname{Ir}(111)$ substrate continue to gradually fill in with BDA molecules whereas the regions that are completely encircled by wrinkles do not show any further domain growth as is witnessed in Figs. 5.1(e) and (f).

First, $\mu$ LEED was used to study the molecular structure of BDA on graphene. Shown in Fig. 5.2(a) are patterns that were first recorded on the graphene flakes that support the BDA illustrating that the orientation of the flakes is aligned to the $\operatorname{Ir}(111)$ substrate. A typical $\mu$ LEED pattern of the BDA domains is shown in Fig. 5.2(b). It consists of the specular reflection surrounded by several rings of LEED spots and reveals an ordered molecular structure. Within the aperture that was used to obtain the LEED 
pattern, several different rotational domains are present. The total number of possible orientations could not be determined from our observations. The red lines highlight the unit cell formed by BDA on graphene. The ordered structure of the molecules that we propose from the $\mu$ LEED pattern is shown in Fig. 5.2(c). BDA molecules are self-assembled into linear chains. In these chains, the molecules are linked together via hydrogen bonds formed by the carboxylic end groups. The chains run parallel and neighboring chains are offset with respect to one another. The proposed model is based on our LEED measurement as well as theoretical findings [27]. These suggest that the $\mathrm{OH}$ unit of the molecule is positioned over the center of a carbon ring in the graphene sheet and that the oxygen atom in the $\mathrm{CO}$ unit of the $\mathrm{COOH}$ end group lies in between two carbon atoms. This arrangement of the $\mathrm{COOH}$ end group helps to predict the position of the hydrogen bonds which are formed over the bridge sites on the graphene. A slight variation in unit cell dimensions allows us to find a bridge site for all the hydrogen bonds and a zigzag type arrangement of the unit cells emerges. The BDA molecules and the chains are positioned in the diagonal direction of the unit cells. In this arrangement, the hydrogen bonds exist with variable lengths and/or individual molecules are contracted or stretched. The dimensions of the unit cell vectors are $0.604 \pm 0.002 \mathrm{~nm}$ and $0.846 \pm 0.003 \mathrm{~nm}$ in length at an angle $\gamma$ of $39.7^{\circ}$. The area $b$ of one unit cell is equal to $0.322 \pm 0.005 \mathrm{~nm}^{2}$. Unlike previous studies $[11,12]$, because of the $\pi-\pi$ repulsive interaction between the core of the BDA molecule and graphene, the BDA molecules are not lying flat on the graphene [27]. The aromatic rings in the molecules are tilted with respect to the surface to minimize the energetic cost of the $\pi-\pi$ repulsion. The carboxylic end groups have a dual function. They are not only responsible for the intermolecular hydrogen bonding that stabilizes the chain structure but also for the molecule-surface bonding. It is important to note that the adsorption of molecules on graphene reduces the strength of intermolecular hydrogen bonds $[27,28]$. The arrangement of the BDA molecules in chains is similar to what is found for BDA/Au(111) and BDA/HOPG $[12,10]$. The temporal evolution of the BDA coverage from a wrinkle bounded region at the center and at the edge of the flake, indicated by the red and blue dashed lines in Fig. 5.1(a), were measured and are shown in Fig. 5.3. In what follows, we shall refer to these as a center and an edge region, respectively.

After the shutter of the evaporator is opened, it takes approximately $50 \mathrm{~s}$ before BDA domains nucleate in either region. We interpret this time as the time that is required to attain a sufficiently high supersaturation of BDA molecules to start the nucleation of BDA domains. After this time, nucleation of BDA domains occurs. The uncovered area of the center region is found to decrease linearly with time and remains constant after the deposition is stopped after $304 \mathrm{~s}$. However, the uncovered area of the edge region shows more complex behavior. The rate at which the uncovered area of the edge region fills in with BDA is initially approximately equal to that of the center region. Later, it is observed to increase with time and becomes greater than that of the center region. When deposition is stopped after $304 \mathrm{~s}$, the uncovered area of the center region remains constant whereas that of the edge regions slowly continues to decrease at a modest rate, illustrated by the arrows in Figs. 5.1(d), (e), and (f).

The gradual growth of BDA domains in edge regions after ceasing deposition and 


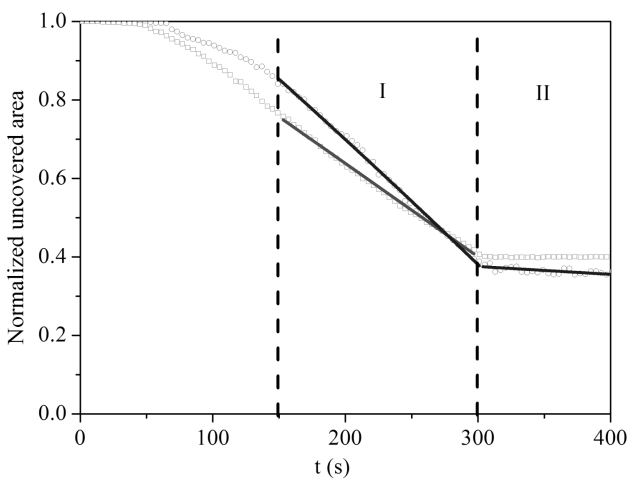

Figure 5.3:

The relative, uncovered area of two graphene regions as a function of deposition time. The red and blue data points were taken from the corresponding areas in Fig. 5.1(a). The solid lines are fits to the data using a linear fit and Eqn. (7.2) in the region I. In region II, solid line is fit to the data using linear fit.

the absence of identical growth in center regions allows us to draw two conclusions. First, there is a net flow of molecules from $\operatorname{Ir}(111)$ onto the graphene at $347 \mathrm{~K}$. Second, there is no net flow of molecules from center regions to edge regions and vice versa. The latter implies that the wrinkles in the graphene sheets effectively block the diffusion of molecules across BDA domains and center regions can effectively be considered as isolated regions for the purpose of BDA deposition and diffusion. A linear fit of the rate at which the uncovered area of the center region decreases in Fig. 5.3, yields a deposition rate, $F$, of $7.38 \pm 0.18 \times 10^{-3} \mathrm{~nm}^{-2} \mathrm{~s}^{-1}$.

The rate at which the uncovered area of edge regions decreases is not constant indicating that the net flow of molecules from $\operatorname{Ir}(111)$ onto the graphene also changes as a function of time. To estimate the rate at which BDA molecules move from $\operatorname{Ir}(111)$ onto graphene, the intensity of the specularly reflected electrons was measured from LEEM images and is shown in Fig. 5.4(a). The intensity measured from the $\operatorname{Ir}(111)$ surface shows a very sharp decrease with time whereas the intensity from the graphene regions decreases only by a minimal amount. We interpret the decrease in reflected intensity on graphene in terms of diffuse scattering caused by an increasing density of admolecules [29]. This interpretation is justified by the LEEM I(V) curves shown in Fig. 5.4(b). They show that a small difference in reflectivity exists at an electron energy of $1.5 \mathrm{eV}$ for clean and BDA covered graphene. The intensity variations on graphene are described with linear fits over three different regions. The linear decay of intensity in region I is due to the diffuse scattering of the admolecules as the concentration of admolecules on graphene gradually increases. Further intensity decay in region II is caused by a work function change that is induced by the close presence of BDA domains which have a lower reflectivity at $1.5 \mathrm{eV}$, see Fig. 5.4(b). The intensity remains constant after ceasing the BDA deposition, as seen in region III. The deposition of BDA molecules on $\operatorname{Ir}(111)$ significantly lowers its work function as well. The onset 

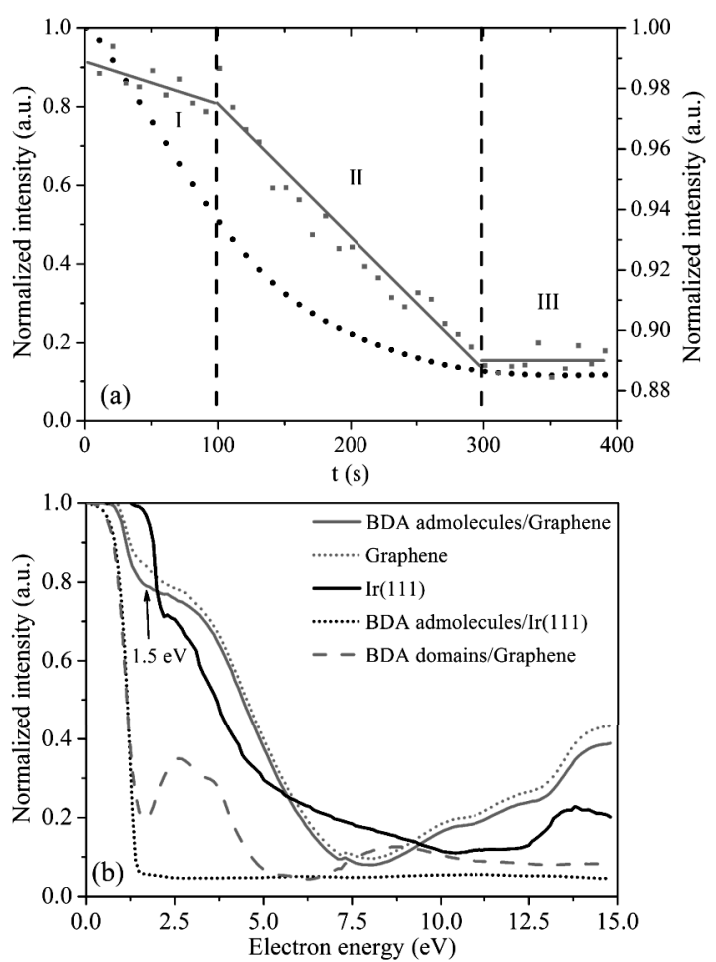

Figure 5.4:

(a) Bright field intensity as a function of deposition time measured on the Ir(111) surface (black solid circles) and graphene (red solid squares) at an energy of $1.5 \mathrm{eV}$. The solid red lines are fits to the data using a linear fit to highlight three regions marked as I, II, and III. Left and right y-axes indicate the intensity of the Ir(111) surface and graphene, respectively. (b) LEEM $I(V)$ characteristics of Ir(111) (solid black line), Ir(111) with BDA admolecules (dotted black line), graphene (dotted red line), graphene with BDA admolecules (solid red line), and BDA domains on graphene (dashed red line). The measured intensities are normalized to the intensity at $-2.0 \mathrm{eV}$.

of total reflection for electrons impinging on the BDA covered $\operatorname{Ir}(111)$ is shifted to the left in the graph of Fig. 5.4(b). This yields a large decrease of the measured bright field intensity in LEEM images. Using the previously measured deposition rate, the density of admolecules can be directly correlated to the reflected intensity on both graphene and $\operatorname{Ir}(111)$. The mechanism through which BDA molecules are able to transfer from $\operatorname{Ir}(111)$ to the BDA domains on the graphene flakes can be further illucidated by examining the temporal evolution of the uncovered area of the edge region which is marked in Fig. 5.1(a). In Fig. 5.5, this area is plotted for $t>304 \mathrm{~s}$, after the deposition of BDA molecules from the vapor phase has already been stopped. The slope of the curve in Fig. 5.5 decreases with time indicating that the rate at which the area of the edge region fills in increases with time. Fig. 5.6 plots the decay rate of the open area of the edge region versus time. The decay rate of the open area is found to increase linearly with time for $t>1000 \mathrm{~s}$. In the absence of a significant temperature variation, a change 


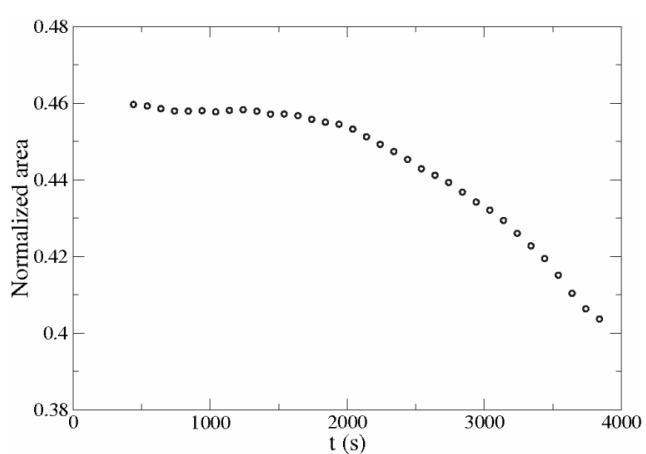

Figure 5.5:

The relative uncovered area of the edge region indicated in Fig. 5.1(a) as a function of time. In the absence of a temperature variation, the rate at which the edge region fills in, is observed to increase with time.

Figure 5.6:

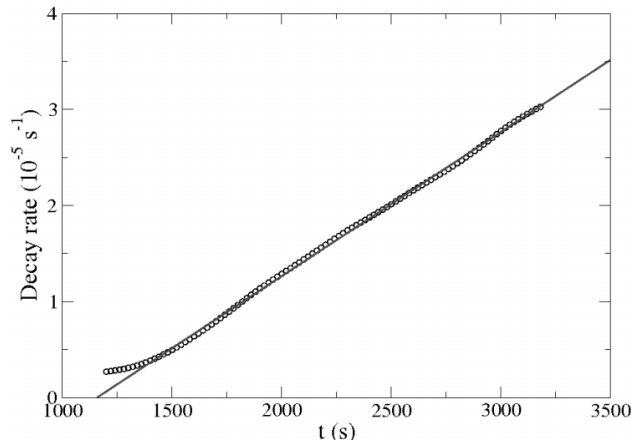

The rate at which the open area of the edge region decays versus time. For $t>1000 \mathrm{~s}$, a linear increase in the decay rate is observed.

in the rate at which edge regions fill in, is surprising. A potential explanation for this behavior is that the fraction of the boundary of the edge region which is open towards the Ir substrate and available for the BDA molecules to ascend onto the graphene, is changing as a function of time. This would imply that BDA molecules can only transfer from the Ir substrate onto a graphene flake at selected sites along the boundary, e.g. at kink sites in the graphene edges. Given that graphene is stable at the temperature of the experiment, $347 \mathrm{~K}$, a change in the physical structure of the edge of the uncovered graphene flake can be ruled out. Instead, we find that the changing decay rate directly correlates with the fraction of the boundary that is decorated by BDA domains on the graphene side of the flake edge. Fig. 5.7 shows the length of the edge of the graphene flake which is part of the blue region of Fig. 5.1 and decorated by BDA domains on the graphene side of the edge. The linear variation that is observed in the decay rate of the area is also found in the length of the edge which is decorated by BDA domains. What this means is that not only the wrinkles in the graphene are effective barriers for the BDA to diffuse across the graphene, also the flake edge constitutes a significant 
diffusion barrier for the BDA to make its way onto the graphene flake. Only with the assistance of BDA molecules which already decorate the top side of a flake edge, are BDA molecules able to make their way onto a graphene flake at $347 \mathrm{~K}$. The net growth

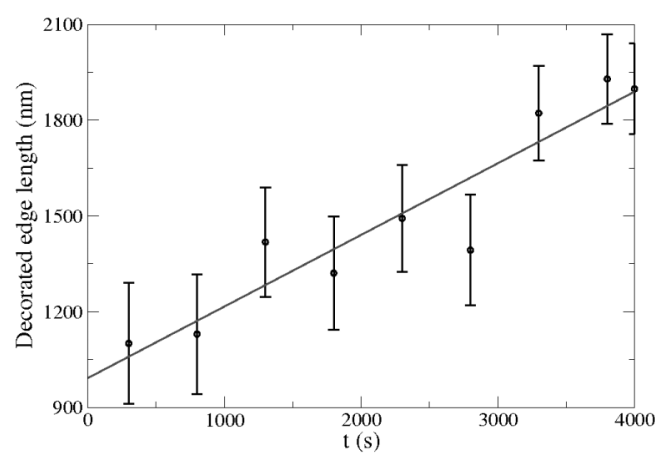

Figure 5.7:

The length of the flake edge that is decorated by BDA domains at the top of the edge plotted versus time.

rate of $\mathrm{BDA}$ domains in edge regions can now be accurately modeled to fit the blue curve of Fig. 5.3. It is equal to the contribution from the vapor phase plus the product of the difference in density of admolecules adjacent to an $\operatorname{Ir}(111)$-graphene boundary multiplied by the hopping rate of molecules across the length of boundary which is available for the BDA to ascend the graphene. The normalized, uncovered area of an edge region is given by

$$
A(t)=1-b \int_{0}^{t} F+F_{\text {boundary }}\left(t^{\prime}\right) d t^{\prime},
$$

where $A$ is the normalized area, and $F_{\text {boundary }}$ is the additional rate at which the edge region fills in with BDA due to molecules crossing the edge of the graphene boundary. The nonlinear evolution of the uncovered area of the edge region which is shown in Fig. 5.3, can now be understood. $F_{\text {boundary }}(t)$ varies significantly with time. When BDA forms domains on graphene, the density of admolecules on graphene has become sufficiently supersaturated for nucleation to occur. The equilibrium concentration of BDA molecules on $\operatorname{Ir}(111)$ is, however, much higher and takes significantly longer to reach as witnessed from Fig. 5.4(a). When the concentration of admolecules on $\operatorname{Ir}(111)$ becomes substantial, $F_{\text {boundary }}(t)$ becomes significant and the blue curve in Fig. 5.3 begins to curve down. Including the contribution of molecules arriving from the vapor phase, the growth of BDA domains in edge regions can be written as

$$
A(t)=1-b F t-\frac{b \sigma a(t) F}{2 l} t^{2}
$$

where $l$ is the length of the boundary of the edge region facing $\operatorname{Ir}(111), a(t)$ is the length of this boundary that is decorated by BDA domains, and $\sigma$ is the hopping rate at which the BDA decorated graphene edge is crossed. All the parameters in Eqn. (7.2) are known except the rate, $\sigma$. The value for the hopping rate that is extracted from a 
quadratic fit to the blue line in region II of Fig. 5.3 is $6.4 \pm 0.3 \times 10^{-3} \mathrm{~s}^{-1}$ for $347 \mathrm{~K}$.

To further expose the behavior of BDA on graphene as a function of temperature, two separate experiments at lower sample temperature (room temperature), shown in Figs. 5.8(a) and (b) and a higher sample temperature, 370 K, shown in Figs. 5.8(c) and (d), were performed. At room temperature, BDA molecules are observed to decorate
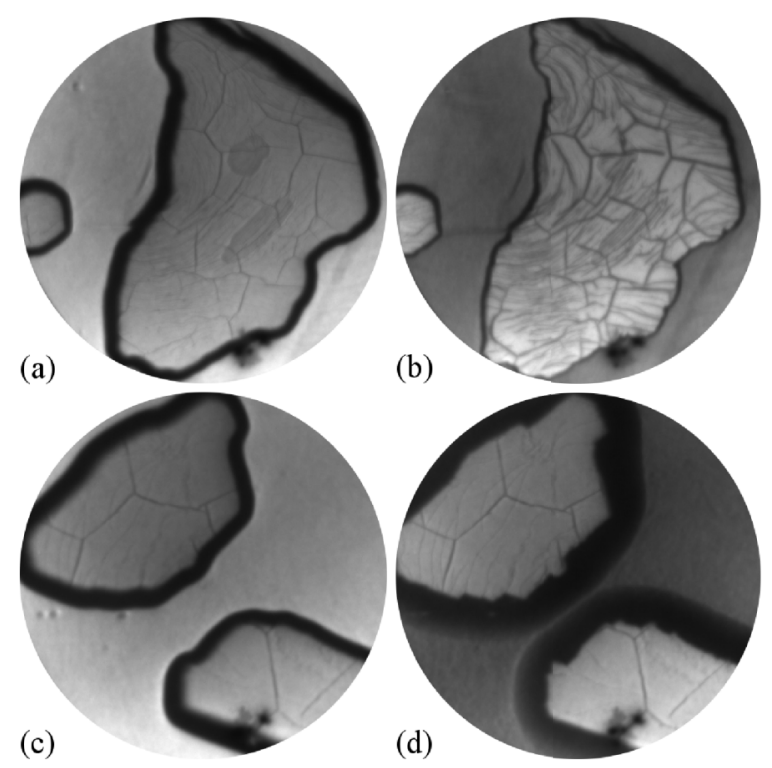

Figure 5.8:

(d)

Time indicated for the subsequent panels are measured with respect to the start of BDA deposition. Two graphene flakes are shown on the Ir(111) surface prior to exposure to BDA. Wrinkles and the contours of the underlying Ir(111) surface steps are visible on the monolayer thick graphene flakes. $(\mathbf{a}, \mathbf{t}=\mathbf{0} \mathbf{s}) 10 \mu \mathrm{m}$ FoV bright-field LEEM image acquired at an electron energy of $1.5 \mathrm{eV}$ and room temperature. $(\mathbf{b}, \mathbf{t}=\mathbf{2 2 0 0} \mathrm{s}) B D A$ molecules decorate the graphene wrinkles and steps. $(\mathbf{c}, \mathbf{t}=\mathbf{0} \mathbf{s}) 6 \mu \mathrm{m}$ FoV bright-field LEEM image acquired at an electron energy of $1.5 \mathrm{eV}$ and temperature of $370 \mathrm{~K} .(\mathbf{d}, \mathbf{t}=\mathbf{5 8 0} \mathrm{s})$ The BDA molecules exclusively form domains on the Ir(111) that extend outwards from the boundaries of the graphene flakes.

graphene wrinkles and protrusions in the graphene flake formed by the Ir steps underneath it. The lower mobility of BDA at this temperature causes the substrate steps to be included with the set of defects on which the BDA domains nucleate. At $370 \mathrm{~K}$, BDA molecules do not decorate either substrate steps or wrinkles. Instead, they show a strongly reduced affinity for graphene and decorate the edges of the graphene flakes on the Ir side of the edge, see Figs. 5.8(c) and (d). This implies that at $370 \mathrm{~K}$, the diffusion of BDA across the graphene edge that was previously observed at $347 \mathrm{~K}$, is in fact reversed. $\mu$ LEED patterns were recorded on the BDA domains that circumfere the graphene flake boundaries but no discernable LEED pattern could be observed from the BDA domains implying a disordered structure of the BDA in those regions.

We have further investigated the orientation of the domains in the BDA networks on graphene by recording dark field LEEM images. Fig. 5.9 shows a $\mu$ LEED pattern and 
images that were recorded by using different spots of the LEED pattern. Figs. 5.9(c)

\section{Figure 5.9:}

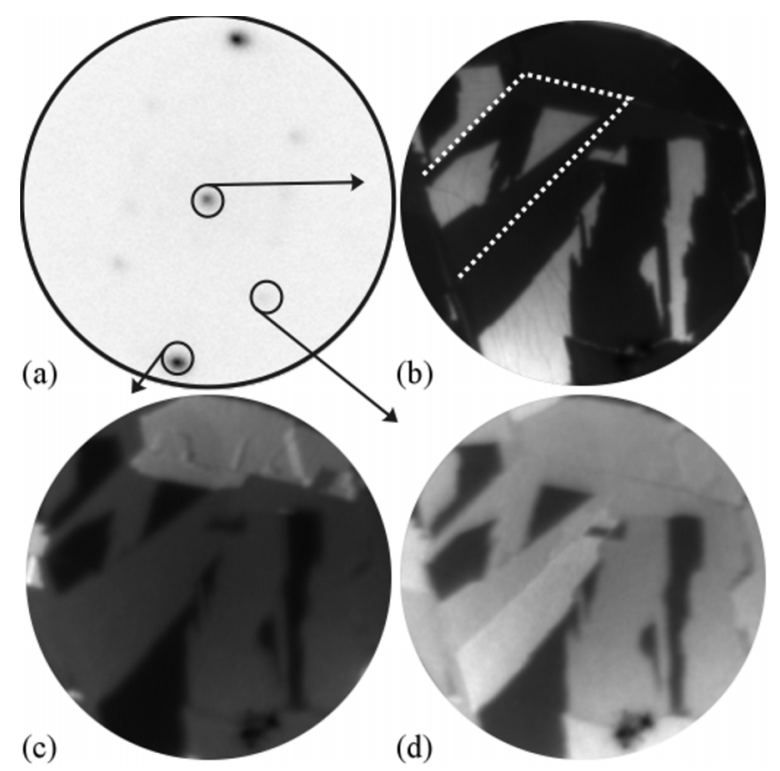

(a) A $\mu L E E D$ pattern of the graphene flake shown in panel (b). (b) A FoV of $6 \mu \mathrm{m}, 5.3 \mathrm{eV}$ LEEM bright field image of a BDA covered graphene flake. The dark areas in the image are $B D A$ domains and bright areas are graphene patches. Dashed white lines in the figure mark the position of wrinkles in the graphene. Panels $(\mathbf{c})$ and $(\mathbf{d})$ are the images recorded in dark field mode using the spots that are indicated by the black circles. In all these images, graphene patches appear dark and BDA domains appear at various levels of contrast depending on the spot used.

and (d) are the dark field LEEM images. In an ideal dark field image, only one single orientation is bright and the rest are dark. This is not always the case in our images because of the finite aperture size and the distribution of orientations of BDA domains present on the graphene flakes. The dotted lines in Fig. 5.9(b) highlight three sides of an area bounded by wrinkles. The domains, which nucleated on the upper side of the marked area maintain their orientation across the wrinkle as shown in Fig. 5.9(c). However, it is also observed that domains that formed on the right hand side of the marked areas have a different orientation with respect to each other on either side of the wrinkle, see Fig. 5.9(d).

Post-deposition annealing of the structures shown in Figs. 5.10(a-c) resulted in decay of the BDA domains. The domains are immobile and remain intact up to a temperature of $360 \mathrm{~K}$. A further increase in temperature leads to a slow decay of the domains. The domains eventually vanished at $370 \mathrm{~K}$ shown in Fig. 5.10(c). If the surface temperature is then reduced to $347 \mathrm{~K}$ as shown in Fig. 5.10(d), nucleation and growth of BDA domains is again observed. This demonstrates that the BDA molecules do not evaporate during the annealing, instead dissociate from the domains that they formed at lower temperature. The BDA chain structure of the domains is stabilized by in- 

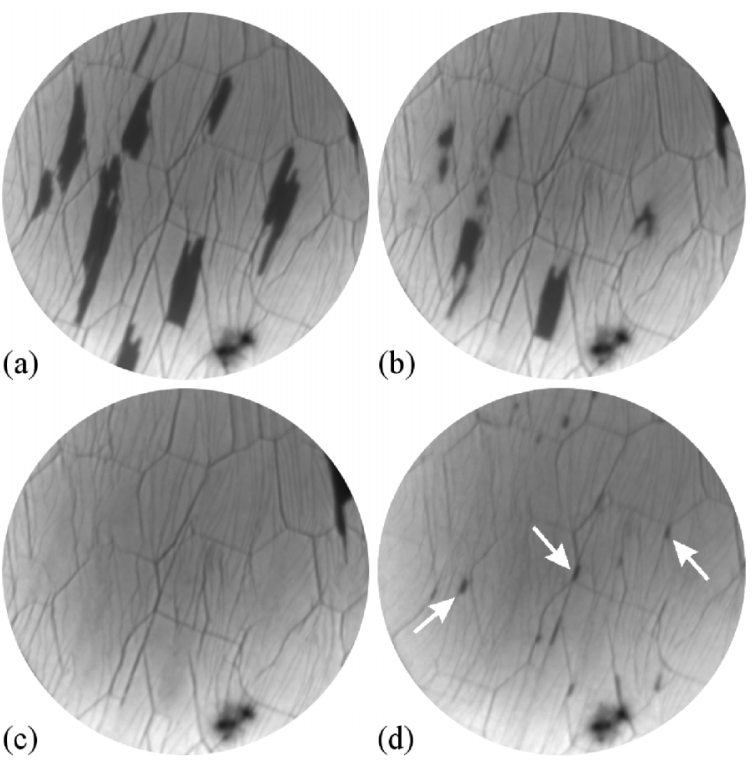

Figure 5.10:

(c)

(d)

(a, T = 346 K) $6 \mu \mathrm{m}$ FoV bright-field LEEM images acquired at an electron energy of $1.5 \mathrm{eV}$ on a graphene flake that is $30^{\circ}$ rotated with respect to the $\operatorname{Ir}(111)$ substrate. BDA domains are grown by deposition. $(\mathbf{b}, \mathbf{T}=\mathbf{3 6 8} \mathbf{K})$ The domains decay with an increase of the graphene surface temperature. $(\mathbf{c}, \mathbf{T}=\mathbf{3 7 0} \mathbf{K})$ All BDA domains have now completely vanished. $(\mathbf{d}, \mathbf{T}=\mathbf{3 4 7} \mathbf{K})$ $B D A$ domains reappear upon lowering of the surface temperature.

termolecular hydrogen bonds. As temperature is increased to a value where the bond energy can be overcome, the BDA chains will gradually fall apart. Assuming thermally activated behavior, an estimate of the pre-factor is needed to determine the activation energy for the breaking of the hydrogen bonds. The breakup of linear chains of similar 4-[trans-2-(pyrid-4-yl-vinyl)] benzoic acid (PVBA) molecules [30] has yielded a prefactor of $10^{10.3 \pm 0.4} \mathrm{~s}^{-1}$. The bonding between PVBA molecules is very similar to that between BDA molecules. Both molecules bond end-to-end by forming hydrogen bonds from carboxyl end groups and experience a weak attractive lateral interaction through $\pi$-bonds. Using this pre-factor, we derive an activation energy of $0.73 \pm 0.03 \mathrm{eV}$ for the breaking of the intermolecular hydrogen bonds which is marginally lower than the value obtained for PVBA. We ascribe the difference to the different substrates used, with the bonding on graphene expected to be weaker.

In a final LEEM experiment, a closed BDA monolayer was formed on top of a graphene flake, shown in Fig. 5.11. The white arrows indicate the position of graphene wrinkles (appearing darker than the BDA film) and black arrows indicate domain boundaries (appearing brighter than the BDA film). The domain boundaries do not have a specific orientation since they result from the coalescence of different rotational domains, which in turn results from the specific configuration of wrinkles that was present prior to the deposition. Domain boundaries are observed to run both parallel (at the lower of the two white arrows) and across (in the far right of the image) wrin- 


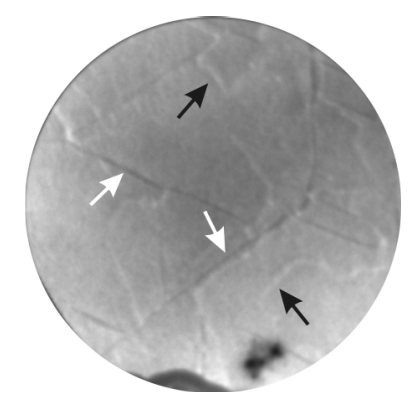

Figure 5.11:

A $4 \mu \mathrm{m}$ FoV bright-field LEEM image of a fully BDA covered graphene flake measured at an electron energy of $1.5 \mathrm{eV}$. The substrate step structure is no longer visible. White arrows indicate wrinkles and black arrows indicate domain boundaries. The domain boundaries occur at the position where different rotational domains coalesce during growth.

kles in Fig. 5.11. Nucleation of a second layer of BDA on the graphene flakes was not observed during or after the completion of the first layer.

\subsection{Discussion}

The observations of Fig. 5.1 confirm that, at $347 \mathrm{~K}$, BDA molecules are mobile on graphene and diffuse over large distances. They are not hindered by the bumps created by the $\operatorname{Ir}(111)$ steps and reach the wrinkles where they nucleate BDA domains as shown in Fig. 5.2, illustrating the important role that wrinkles play in the nucleation of BDA domains. Nucleation ceases when there are no empty wrinkles left on the graphene.

We now speculate on the reason for the preferred adsorption of BDA molecules on wrinkles. Steps on crystal surfaces are well known to constitute a preferential adsorption site for adatom species [31]. The local curvature of the graphene sheets that occurs at wrinkles could effectively turn the wrinkles into step sites. A second possible explanation could be that the local delamination of the graphene film from the Ir substrate around wrinkles leads to a stronger bonding of BDA to the graphene in the delaminated areas. The spatial extent of the wrinkle, however, is laterally limited to only a few $\mathrm{nm}$. Instead, our observation that the diffusion of BDA molecules is not hindered by steps protruding from the $\operatorname{Ir}(111)$ substrate as well as the observation that BDA domains nucleate in graphene regions with a high curvature, hints at a different mechanism. The formation of wrinkles has been demonstrated to lead to a local variation of strain in the graphene film particularly on and near the wrinkles themselves [20]. The local variations of strain in the graphene film will lead to variations in binding energy of the BDA molecules and cause them to preferentially attach to these energetically favored adsorption sites [32]. On the other hand, local variation of surface strain modifies the diffusion of adsorbates. A location with a lower adsorbate mobility is a preferred nucleation site for the domains [33]. This effect is directly probed by following the heterogeneous nucleation of the adsorbates as was done in Fig. 5.1 and is likely to be the reason for BDA to be unable to transgress the wrinkles in the graphene.

A puzzling observation that still remains to be discussed is that at $347 \mathrm{~K}$ BDA flows 
from Ir to graphene whereas the situation is reversed at $370 \mathrm{~K}$ and the BDA prefers Ir over graphene. This surprising reversal of affinity for both types of surface must have an energetic origin rather than a kinetic one. We first note that the reversal of the BDA transport from and to graphene coincides with the breaking and re-establishing of the hydrogen bonds which are responsible for the vanishing of BDA domains on extended graphene areas upon heating from $347 \mathrm{~K}$ to $370 \mathrm{~K}$ and their reappearance upon cooling from $370 \mathrm{~K}$ to $347 \mathrm{~K}$. Disordered BDA on $\operatorname{Ir}(111)$ must have a higher free energy than the hydrogen bond induced ordered domains that BDA forms on graphene at $347 \mathrm{~K}$. It is also known that individual BDA molecules have a higher affinity for Ir than for graphene since BDA evades the graphene flakes at $370 \mathrm{~K}$ in favour of Ir. These ingredients provide the key for a possible explanation. We suggest that strain governs, whether or not ordered BDA domains form on graphene. The stronger bonding of BDA on the mismatched Ir imposes substantial strain which can only be relieved by accommodating the BDA in ordered domains on graphene, with its lesser affinity and probably smaller mismatch as driving factors.

\subsection{Conclusions}

BDA has been employed as a prototype molecule to study the formation of molecular films on metal supported graphene. Our bright field LEEM measurements reveal that the morphology that develops as graphene is exposed to BDA is determined by defects in the graphene film. Wrinkles are the preferred sites for the nucleation of BDA domains. The origin of the inhomogeneous nucleation of BDA domains is speculated to be the strain relaxation that occurs at the wrinkles. $\mu$ LEED measurements reveal that the BDA domains that form are an ordered, hydrogen bond stabilized, chain structures. Dark field LEEM measurements reveal that a continuous film of BDA consists of numerous different rotational domains that transgress wrinkles and bumps originating from substrate steps. Domain boundaries are observed in those locations where growing domains have coalesced. The films that were grown were stable over extended periods of time at room temperature and slightly above. Above $350 \mathrm{~K}$, decay of the BDA domains was observed. The edges of a graphene flake were shown to act as an additional source of molecules for extended times even when BDA was no longer deposited from the vapor phase. BDA domains that were present at the top side of a graphene flake edge was shown to assist in the upwards diffusion of BDA onto the graphene flake.

The use of graphene as a substrate for self-assembly of molecular networks opens up a new applications area by combining the principles of two-dimensional organic self-assembly with the remarkable electronic properties of graphene. It offers enormous possibilities when using custom-made molecules that alter the molecule-molecule or molecule-graphene interaction. 


\section{Bibliography}

[1] A.H. Castro Neto, F. Guinea, N.M.R. Peres, K.S. Novoselov, and A.K. Geim, Rev. Mod. Phys. 81 (2009), 109.

[2] R.M. Tromp and J.B. Hannon, Phys. Rev. Lett. 102 (2009), 106104.

[3] A.T. N'Diaye, J. Coraux, T.N. Plasa, C. Busse, and T. Michely, New J. Phys. 10 (2008), 043033.

[4] J. Coraux, A.T. N'Diaye, M. Engler, C. Busse, D. Wall, N. Buckanie, F.-J. Meyer zu Heringdorf, R. van Gastel, B. Poelsema, and T. Michely, New J. Phys. 11 (2009), 023006.

[5] R. van Gastel, A.T. N’Diaye, D. Wall, J. Coraux, C. Busse, N.M. Buckanie, F.-J. Meyer zu Heringdorf, M. Horn von Hoegen, T. Michely, and B. Poelsema, Appl. Phys. Lett. 95 (2009), 121901.

[6] P.W. Sutter, J.-I. Flege, and E.A. Sutter, Nat. Mater. 7 (2008), 406.

[7] E. Loginova, N.C. Bartelt, P.J. Feibelman, and K.F. McCarty, New J. Phys. 10 (2008), 093026.

[8] A. Reina, X. Jia, J. Ho, D. Nezich, H. Son, V. Bulovic, M.S. Dresselhaus, and J. Kong, Nano Lett. 9 (2009), 30.

[9] Q.H. Hwang and M.C. Hersam, Nat. Chem. 1 (2009), 206.

[10] C. Heininger, L. Kampschulte, W.M. Heckl, and M. Lackinger, Langmuir 25 (2009), 968.

[11] S. Stepanow, N. Lin, F. Vidal, A. Landa, M. Ruben, J.V. Barth, and K. Kern, Nano Lett. 5 (2005), 901.

[12] N. Zhu, T. Osada, and T. Komeda, Surf. Sci. 601 (2007), 1789.

[13] A. Dmitriev, N. Lin, J. Weckesser, J.V. Barth, and K. Kern, J. Phys. Chem. B 106 (2002), 6907.

[14] D. Payer, A. Comisso, A. Dmitriev, T. Strunskus, N. Lin, C. Wöll, A. DeVita, J.V. Barth, and K. Kern, Chem. Eur. J. 13 (2007), 3900.

[15] T. Suzuki, T. Lutz, D. Payer, N. Lin, S.L. Tait, G. Costantini, and K. Kern, Chem. Phys. 11 (2009), 6498.

[16] S. Stepanow, M. Lingenfelder, A. Dmitriev, H. Spillmann, E. Delvigne, N. Lin, X. Deng, C. Cai, J.V. Barth, and K. Kern, Nat. Mater. 3 (2004), 229.

[17] S. Griessl, M. Lackinger, M. Edelwirth, M. Hietschold, and W.M. Heckl, Single Mol. 3 (2002), 25.

[18] E. Bauer, Surf. Rev. Lett. 5 (1998), 1275. 
[19] E. Loginova, S. Nie, K. Thürmer, N.C. Bartelt, and K.F. McCarty, Phys. Rev. B 80 (2009), 085430.

[20] A.T. N’Diaye, R. van Gastel, A.J. Martínez-Galera, J. Coraux, H. Hattab, D. Wall, F.-J. Meyer zu Heringdorf, M. Horn-von Hoegen, J.M. Gómez-Rodríguez, B. Poelsema, C. Busse, and T. Michely, New J. Phys. 11 (2009), 113056.

[21] K. Xu, P. Cao and J.R. Heath, Nano Lett. 9 (2009), 4446.

[22] K.S. Kim, Y. Zhao, H. Jang, S.Y. Lee, J.M. Kim, K.S. Kim, J.-H. Ahn, P. Kim, J.-Y. Choi, and B.H. Hong, Nature 457 (2009), 706.

[23] A.N. Obraztsov, E.A. Obraztsova, A.V. Tyurnina, and A.A. Zolotukhin, Carbon 45 (2007), 2017.

[24] J.S. Chae et al., Adv. Mater. 21 (2009), 2328.

[25] P. Sutter, J.T. Sadowski, and E. Sutter, Phys. Rev. B 80 (2009), 245411.

[26] X. Li, W. Cai, J. An, S. Kim, J. Nah, D. Yang, R. Piner, A. Velamakanni, I. Jung, E. Tutuc, S.K. Banerjee, L. Colombo, and R.S. Ruoff, Science 324 (2009), 1312.

[27] A. Rochefort and J.D. Wuest, Langmuir 25 (2009), 210.

[28] A. Rochefort, private communication.

[29] J. de la Figuera, N.C. Bartelt, and K.F. McCarty, Surf. Sci. 600 (2006), 4062.

[30] J. Weckesser, J.V. Barth, and K. Kern, J. Chem. Phys. 110 (1999), 5351.

[31] L.W. Bruch, R.D. Diehl, and J.A. Venables, Rev. Mod. Phys. 79 (2007), 1381, and references therein.

[32] M. Gsell, P. Jakob, and D. Menzel, Science 280 (1998), 717.

[33] H. Brune, K. Bromann, H. Röder, K. Kern, J. Jacobsen, P. Stoltze, K. Jacobsen, and J. Nørskov, Phys. Rev. B 52 (1995), 14380. 


\section{Smooth growth of 6P films on graphene for high efficiency electronics}

The growth of high-quality films of conjugated molecules with smooth interfaces is important to assist the advent of organic electronics. In previous chapters, we have investigated the growth of BDA molecules on different surfaces. Here, we report on the layer-by-layer growth of the organic semiconductor molecule, 6P, on the transparent electrode material graphene. 6P has a longer backbone than BDA and is anticipated to form more complex structures when grown on flat, defect-free substrates. LEEM and $\mu L E E D$ reveal the morphological and structural evolution of the thin film, the layerby-layer growth of $6 P$ on graphene*.

*Published in Nano Lett. 11 (2011), 333. 


\subsection{Introduction}

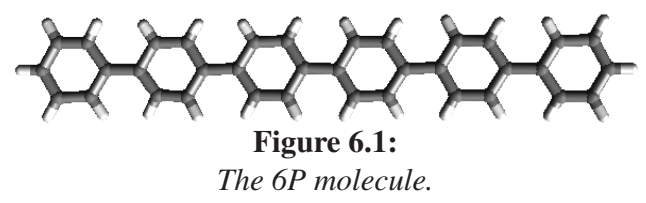

Smooth interfaces are a prerequisite for future, high performance, and low cost organic electronic devices [1] based on small conjugated molecules. The quality of the first few layers is of critical importance since all important charge transport processes are confined to the first 2 or 3 monolayers [2]. However, often these films grow in a three dimensional manner resulting in rough surfaces [3-7]. To obtain sufficient smoothness at the interface, it is a prerequisite that the active region is formed in the layer-by-layer growth mode. The resulting flat interfaces exhibit a lower number of defects and generally yield a higher charge carrier mobility [8-11]. We have achieved this goal for para-sexiphenyl (6P) molecules on graphene. The 6P molecule is depicted graphically in Fig. 6.1. Layer-by-layer growth of lying 6P molecules on metal supported graphene flakes is realized. The formation of several layers has been monitored in situ by means of LEEM. $\mu$ LEED has been used to reveal a bulk-like structure of the submonolayer, monolayer, and multilayer regime. The combination of the established deposition technique OMBE with the unique properties of organic semiconductors and graphene is a viable route for future flexible and cost efficient devices based on small conjugated molecules. On the one hand, $6 \mathrm{P}$ is a blue light emitter with a high charge carrier mobility [12], which makes it well suited for the fabrication of organic light emitting diodes. On the other hand, graphene is a flexible, highly conductive, and transparent electrode material [13, 14], ideally suited as a technological substrate for organic semiconductors $[15,16]$. For the present study, graphene flakes on $\operatorname{Ir}(111)$ were used as they show only weak coupling to the underlying substrate [17] and can be grown in millimeter size [18]. A transparent substrate and layer-by-layer growth of lying molecules are the perfect combination for high output color tunable organic light emitting diodes [19].

\subsection{Experimental}

Single layer graphene sheets were grown on an $\operatorname{Ir}(111)$ surface [20]. The metal crystal was cleaned by high temperature exposure to $\mathrm{O}_{2}$. The graphene layer was then formed by thermal decomposition of $\mathrm{C}_{2} \mathrm{H}_{4}$ on the hot $(875 \mathrm{~K}) \operatorname{Ir}(111)$ surface [18]. The growth of graphene was followed in real-time using PEEM until sufficiently large flakes had formed on the surface. Using $\mu \mathrm{LEED}$, the orientation of the graphene flakes was verified. Only flakes that are aligned with the substrate were selected for analysis during and after the deposition of 6P [18]. The sample was then cooled to $240 \mathrm{~K}$ before deposition of 6P by OMBE from a resistively heated Knudsen-cell evaporator designed for the deposition of organic molecules. 


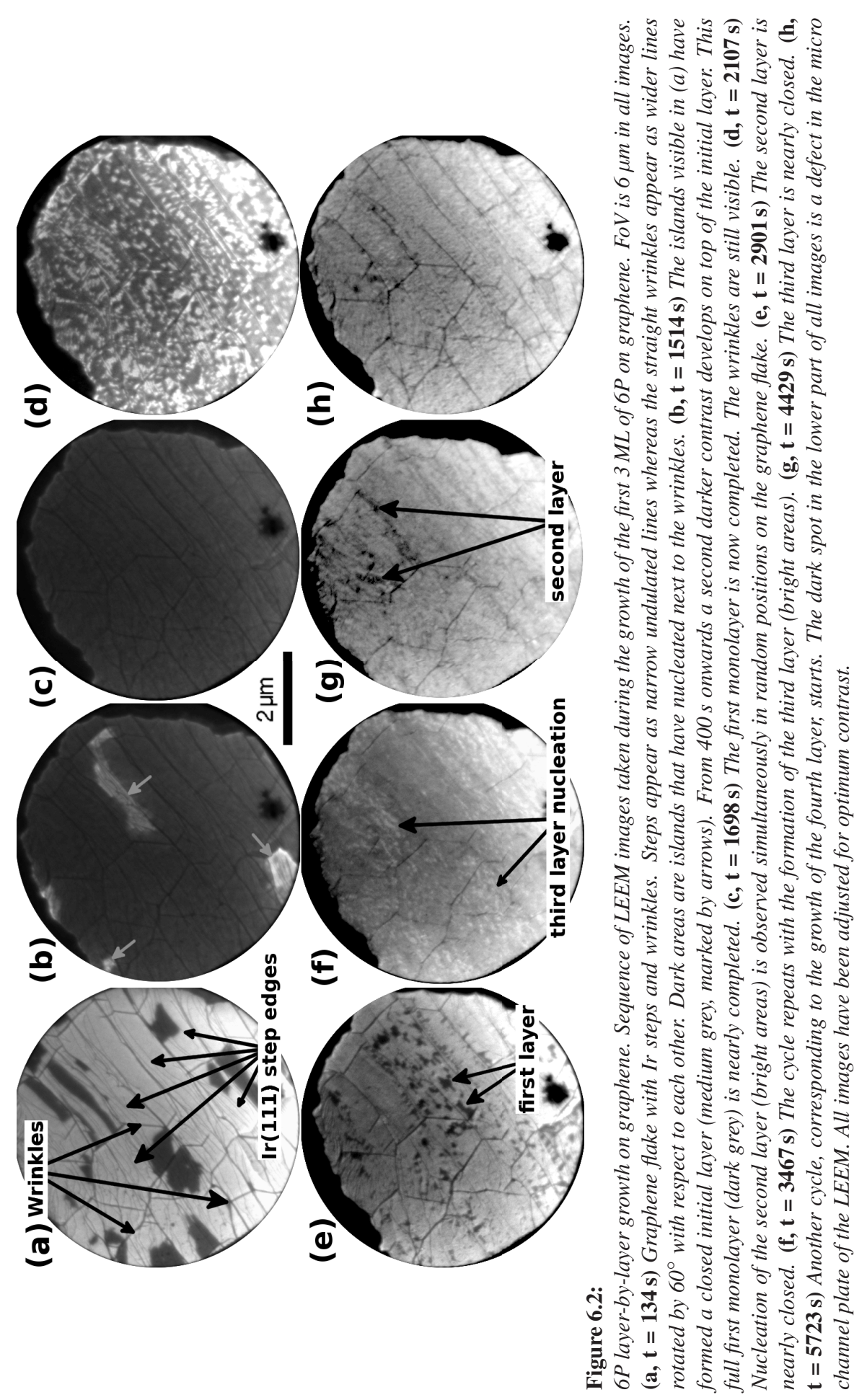




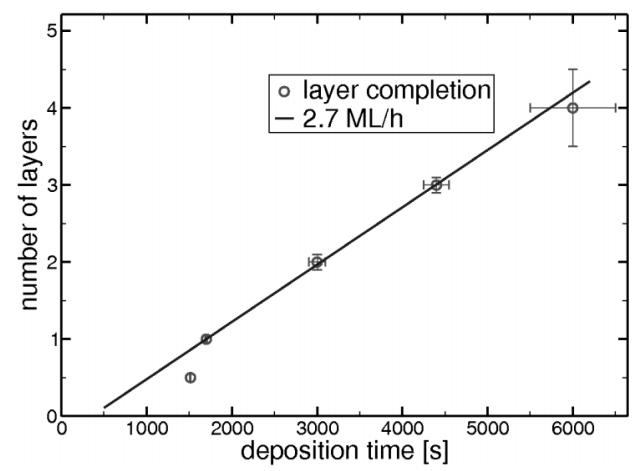

Figure 6.3:

Layer completion times. All but the first data point correspond to the closing of a full monolayer of 6 . From the linear fit (ignoring the first data point), a growth rate of $2.7 \mathrm{ML} / \mathrm{h}$ is obtained.

The sublimation purified source material has been purchased from TCI Europe N.V. Care was taken to remove remaining low boiling point contaminations by a thorough outgassing of the evaporator for several hours prior to the experiment. The film formation was followed in-situ using an Elmitec LEEM III. Images were recorded every second at typical energies of $2 \mathrm{eV}$, well below the band gap of $3.1 \mathrm{eV}$ for $6 \mathrm{P}$. The film structure was investigated using the in situ $\mu$ LEED capabilities of the instrument. All $\mu$ LEED measurements were carried out at the deposition temperature of $240 \mathrm{~K}$ using a field limiting aperture with a projected diameter of $1.4 \mu \mathrm{m}$.

\subsection{P growth}

Fig. 6.2 shows a sequence of LEEM images taken during growth of the first 4 MLs of $6 \mathrm{P}$ on graphene. Fig. 6.2a shows a graphene flake and the first $6 \mathrm{P}$ islands that nucleated after $134 \mathrm{~s}$ of deposition. Thin undulated lines correspond to steps of the supporting $\operatorname{Ir}(111)$ substrate. The more pronounced straight thick lines stem from wrinkles in the graphene layer $[21,22]$ while the islands do not grow over the wrinkles they do cross the steps of the underlying Ir. After roughly $400 \mathrm{~s}$ of growth (not shown) a second, darker level of contrast becomes visible in the center of the existing 6P islands. Eventually, the initial layer (1514 s of 6P growth, medium grey, marked by arrows) has closed (Fig. 6.2b). Only $184 \mathrm{~s}$ later, the second darker area covers the whole surface (Fig. 6.2c). In Fig. 6.2d, bright islands are observed to form. They eventually coalesce (Fig. 6.2e) leading to a uniform contrast. This cycle then repeats with the nucleation of another set of islands (Fig. 6.2f). After some time, the contrast becomes uniform again (see Fig. 6.2g) indicating the completion of the next layer. Fig. 6.2h shows the start of the next repetition of this cycle. Fig. 6.3 shows the temporal distribution of the formation of fully closed layers. Using all but the first data point, which corresponds to the closing of the initial layer, we find a growth rate of $2.7 \mathrm{ML} / \mathrm{h}$. Here, we use the term monolayer for a closed layer of molecules having the final structure. The layerby-layer growth is therefore followed for 4 complete layers, the structure of which will be discussed next. 

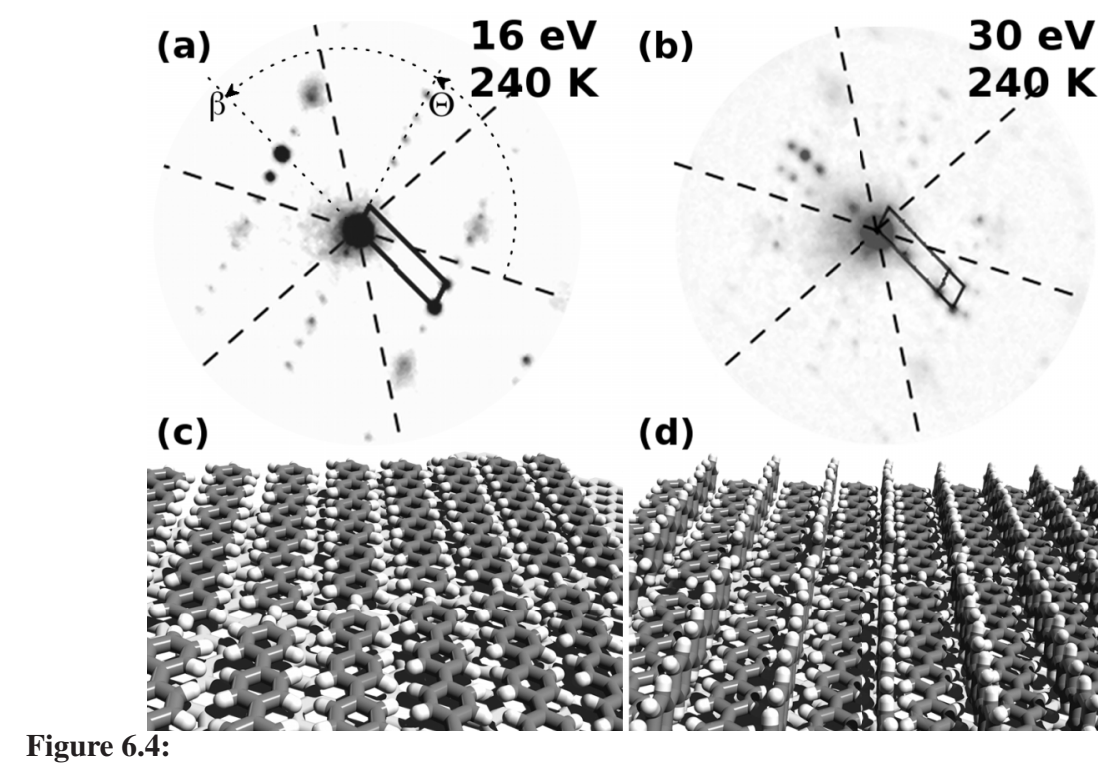

Figure 6.4:

Submonolayer and monolayer structure. (a) $\mu L E E D$ pattern obtained from the first half and, (b) the completed first layer. Dashed lines indicate the [1000] (zigzag) direction of the graphene flake. The angles $\beta$ and $\Theta$ used for the description of the unit cells are indicated. Panels (c) and (d) show the proposed structure of the first half and full first layer. (In (c) and (d), planar molecules are used for clarity).

The structure of the 6P layers was characterized by $\mu$ LEED. Fig. 6.4 shows two LEED patterns obtained during the formation of the first complete layer of $6 \mathrm{P}$ on graphene. The measured unit cell is $a=28.1 \AA$ and $b=6.0 \AA$, with $\beta=79^{\circ}$ and $\Theta=79^{\circ}$ for the initial layer (Fig. 6.4a) that yields a medium grey contrast in Figs. 6.2(a), (b). Here, $a$ and $b$ denominate the long and short unit cell axis, $\beta$ the angle between them and $\Theta$ is the angle between the long side $a$ and the [1000] zigzag direction of the graphene layer. From the size of the unit cell, it is immediately evident that the film is formed by one flat-lying molecule per unit cell, i.e. molecules where the average orientation of the benzene rings is parallel to the substrate. Contrary to what has been observed for 6P on highly oriented pyrolytic graphite [23], the molecules are not aligned with the [1]100] armchair direction of graphene. Instead, a similar, loosely packed open structure with a matrix notation of $\left[\begin{array}{cc}8.7 & 13.0 \\ -1.3 & 1.5\end{array}\right]$ is found (Fig. 6.4c). The long axis (LA) of the molecule is parallel to the [0120] direction of graphene (corresponds to rotation by $11^{\circ}$ with respect to graphite) while the short axis (SA) is $3^{\circ}$ off the $[\overline{2} 0 \overline{1} 0]$ direction. Assuming an on-top position for the first phenyl ring, the fourth phenyl ring will have a similar position. This metastable structure only exists in the initial stage of the formation of a layer. Why it is different might be related to the unique structure of graphene. Little is known about the adsorption of organic molecules on graphene. However, recent studies on benzene adsorption reveal a net Mullikin charge transfer of $0.03 \mathrm{e}$ from the molecule to the graphene [24]. In addition, the same study reveals a weaker binding of benzene to graphene $\left(E_{b}=0.24 \mathrm{eV}\right)$ compared to values 
reported for graphite $\left(E_{b}=0.35 \mathrm{eV}\right)$ [25]. As a first estimate for $6 \mathrm{P}$ on graphene, these values can be multiplied by six to achieve the correct order of magnitude.

To shed more light on the difference in adsorption geometry, some basic molecular dynamic simulations have been performed. Single 6P molecules have been placed on sufficiently large pieces of either single or double layer graphene to simulate graphite. A variant [26] of the Tripos-5.2 force-field [27] has been used for these calculations in combination with the molecular modeling software Avogadro [28]. All four possible configurations have been allowed to relax until the change between two successive steps was less than a fraction of $10^{-8}$ of the total energy. The obtained total energies were compared to the sum of the total energies of the molecule and the substrate. Comparing the calculated binding energies, the case of the LA of $6 \mathrm{P}$ parallel to the $(01 \overline{2} 0)$ direction of graphene $\left(L A_{6 P} \|\left(01 \overline{2} 0_{\text {graphene }}\right)\right)$ is favored (by $\left.\approx 300 \mathrm{meV}\right)$ on graphene while the LA of $6 \mathrm{P}$ parallel to the $10 \overline{1} 0$ direction $\left(L A_{6 P} \|\left(10 \overline{1} 0_{\text {graphite }}\right)\right)$ is favored (by $\approx 100 \mathrm{meV}$ ) for the double layer graphene sheet. These results perfectly agree with the observations by Wang et al. for 6P on graphite, and those made in the current paper for 6P on graphene.

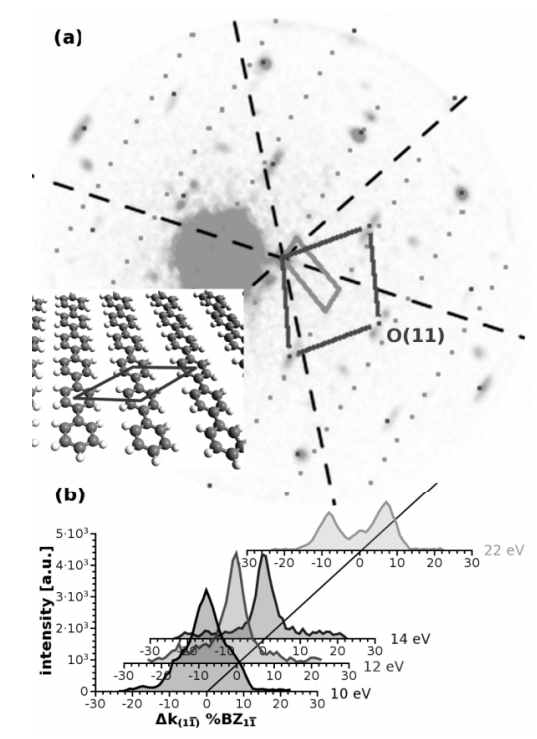

Figure 6.5:

$\mu L E E D$ pattern obtained from a 4.5 ML thick 6P film on graphene. (a) The measured unit cell parameters for the green cell are: $a=26.9 \AA, b=9.2 \AA$, with $\beta=74^{\circ}$ and $\Theta=77^{\circ}$. The red unit cell with a size of $a=6.8 \AA, b=6.4 \AA$, with $\beta=75^{\circ}$ and $\Theta=141^{\circ}$, results from diffraction of the individual phenyl rings. The inset shows the real space structure of the adlayer together with the unit cell (solid red line). The dashed unit cell indicates the resulting unit cell for the molecules. (b) Spot profiles of the $\mathrm{O}(11)$-spot belonging to the red unit cell in panel (a). The profiles for different electron energies have been shifted to enhance visibility.

After roughly $400 \mathrm{~s}$ of $6 \mathrm{P}$ film growth, a second more dense structure starts to form resulting in the LEED pattern, presented in Fig. 6.4b, and corresponding to the dark grey contrast in the LEEM images (see Figs. 6.2b,c). The unit cell size increases to 
$a=28.1 \AA, b=7.5 \AA, \beta=69^{\circ}$, and $\Theta=79^{\circ}$ (matrix notation: $\left[\begin{array}{cc}8.7 & 13.0 \\ -1.7 & 1.9\end{array}\right]$; two molecules per unit cell). The only way to accommodate the additional $6 \mathrm{P}$ molecules is in an edge-on configuration, interdigitating the flat-lying molecules. In addition, some of the former flat-lying molecules will need to tilt into an edge-on configuration as well. The energy gain due to the higher mismatch - compared to the completely flat lying initial layer - is more than compensated by the positive effect of a bulklike arrangement of the molecules already in the first monolayer (Fig. 6.4d). A similar structure and growth mechanism for the first monolayer of $6 \mathrm{P}$ has been observed on $\mathrm{Au}(111)$ [29].

Fig. 6.5a shows a $\mu$ LEED pattern that was obtained from a $4.35 \mathrm{ML} 6 \mathrm{P}$ film on graphene. Two unit cells can be identified. Several weak spots can be attributed to a unit cell with a size of $a=26.9 \AA, b=9.2 \AA$, with $\beta=74^{\circ}$ and $\Theta=77^{\circ}$. A second smaller unit cell has the dimensions: $a=6.8 \AA, b=6.4 \AA$, with $\beta=75^{\circ}$ and $\Theta=141^{\circ}$. Keeping the amount of deposited $6 \mathrm{P}$ in mind, we interpret the former as a bulk continuation of what was observed for the first layer. This unit cell is similar to the surface unit cell of the bulk $6 \mathrm{P}\{1 \overline{11}\}$ plane [30]. This bulk-like unit cell with

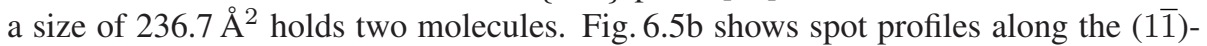
direction of the spot labeled $O(11)$ for different energies (extracted from the $\mu$ LEED patterns). Such spot profiles are the result of a regular step train in two layers, i.e. single layer islands of equal size in a regular arrangement [31]. From the spot splitting, we infer an island size of $28.7 \AA$ implying that the $6 \mathrm{P}$ molecules themselves give rise to the spot-splitting. Therefore, the smaller of the two unit cells is associated with diffraction from the ordered phenyl rings that constitute the $6 \mathrm{P}$ molecule. Using the measured positions for the phenyl rings, a unit cell for the molecules can be derived with a size of $a=29.2 \AA, b=6.3 \AA$, with $\beta=75^{\circ}$ and $\Theta=0^{\circ}$ Here, $\Theta$ is given with respect to the long unit cell axis of the underlying bulk $6 \mathrm{P}$. One molecule is contained in this unit cell which has an area of $162.7 \AA^{2}$ and a matrix notation of $\left[\begin{array}{cc}1.1 & 0 \\ 0 & 0.7\end{array}\right]$ with respect to the underlying $6 \mathrm{P}$. This overlayer is aligned with the underlying bulk $6 \mathrm{P}$ but only every 11 and 7 molecules along the long and short axis direction, respectively, will be in the same position. As a result of the lower molecular density in the adlayer, $50 \%$ of the surface is covered by this adlayer. The measured energy dependence of the spot profile (see Fig. 6.5b) allows to estimate the thickness of the adlayer. Using $2 d=n \lambda$ [32] for the in phase condition (at $14 \mathrm{eV}$ ) and $2 d=\left(n+\frac{1}{2}\right) \lambda$ [32] for the out of phase condition (at $22 \mathrm{eV}$ ), we can calculate a value of $n=2(1.97)$. The resulting spacing between the adlayer and the uppermost flat molecules of the bulk $6 \mathrm{P}$ is then $3.3 \AA$. The distance between two $\{1 \overline{11}\}$ planes in the bulk is $4.6 \AA$ [30]. Fig. 6.6 shows the proposed structure for the full stack of molecules. Four layers of bulk-like $6 \mathrm{P}$ (grey carbon atoms) with the $\{1 \overline{11}\}$ plane parallel to the underlying graphene sheet (light blue carbon atoms for clarity) are covered by an adlayer of only flat lying molecules (orange carbon atoms).

\subsection{Conclusions}

In conclusion, using LEEM, we have demonstrated the growth of atomically smooth layers of the organic semiconductor $6 \mathrm{P}$ on a graphene substrate. Initially, small islands are formed. An open structure consisting of only flat-lying molecules was found as 


\section{Figure 6.6:}

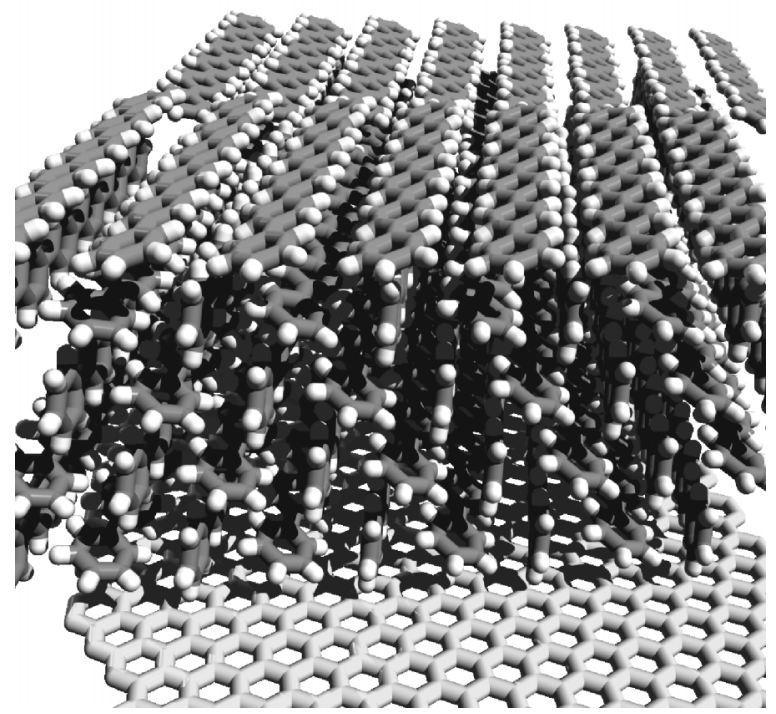

Proposed structure of 6P on metal supported graphene. 4 Layers of bulk-like 6P are deposited with their (111) plane parallel to the graphene substrate (light blue carbon atoms for clarity). The adlayer covering 50\% of the top surface is shown with orange carbon atoms.

an initial structure for the first layer with $\mu$ LEED. This layer then transforms into a complete monolayer through the addition of interdigitating edge-on molecules, which result in a bulk-like arrangement of the molecules. Subsequent layers are formed by a repetition of this cycle, as we find, an adlayer with an open structure similar to what was found for the initial layer covering the surface of thicker films. Up to at least 4.35 ML, the growth continues in this layer-by-layer fashion. This growth mode will lead to films with a high charge carrier mobility and good overall device performance. As such it is an enabler for future organic, flexible, and low cost devices [33]. 


\section{Bibliography}

[1] C.K. Chiang, R.C. Fincher, Y.W. Park, A.J. Heeger, H. Shirakawa, E.J. Louis, S.C. Gau, and A.G. MacDiarmid, Phys. Rev. Lett. 39 (1977), 1098.

[2] A. Dodabalapur, L. Torsi, and H.E. Katz, Science 268 (1995), 270.

[3] A.C. Dürr, F. Schreiber, K.A. Ritley, V. Kruppa, J. Krug, H. Dosch, and B. Struth, Phys. Rev. Lett. 90 (2003), 016104.

[4] S. Kowarik, A. Gerlach, S. Sellner, F. Schreiber, J. Pflaum, L. Cavalcanti, and O. Konovalov, Phys. Chem. 8 (2006), 1834.

[5] T. Mikami and H. Yanagi, Appl. Phys. Lett. 73 (1998), 563.

[6] G. Hlawacek, P. Puschnig, P. Frank, A. Winkler, C. Ambrosch-Draxl, and C. Teichert, Science 321 (2008), 108.

[7] D.M. Smilgies and E.-J. Kintzel, Phys. Rev. B 79 (2009), 235413.

[8] H. Yan, S. Swaraj, C. Wang, I. Hwang, N.C. Greenham, C. Groves, H. Ade, and R.C. McNeill, Adv. Funct. Mater. 40 (2010), 4329.

[9] S.E. Fritz, T.W. Kelley, and C.D. Frisbie, J. Phys. Chem. B 109 (2005), 10574.

[10] S. Steudel, S. De Vusser, S. De Jonge, D. Janssen, S. Verlaak, J. Genoe, and P. Heremans, Appl. Phys. Lett. 85 (2004), 4400.

[11] D. Schumacher and D. Stark, Surf. Sci. 123 (1982), 384.

[12] T. Singh, G. Hernandez-Sosa, H. Neugebauer, A. Andreev, H. Sitter, and N.S. Sariciftci, Phys. Stat. Sol. B 243 (2006), 3329.

[13] A.K. Geim and K.S. Novoselov, Nat. Mater. 6 (2007), 183.

[14] T.J. Echtermeyer, M.C. Lemme, M. Baus, B.N. Szafranek, A.K. Geim, and H. Kurz, IEEE J. EDL 29 (2008), 952.

[15] P. Lauffer, K.V. Emtsev, R. Graupner, T. Seyller, and L. Ley, Phys. Stat. Sol. B 245 (2008), 2064.

[16] Q.H. Wang and M.C. Hersam, Nat. Chem. 1 (2009), 206.

[17] I. Pletikosić, M. Kralj, P. Pervan, R. Brako, J. Coraux, A.T. N’Diaye, C. Busse, and T. Michely, Phys. Rev. Lett. 102 (2009), 056808.

[18] R. van Gastel, A.T. N'Diaye, D. Wall, J. Coraux, C. Busse, N.M. Buckanie, F.J. Meyer zu Heringdorf, M. Horn von Hoegen, T. Michely, and B. Poelsema, Appl. Phys. Lett. 95 (2009), 121901.

[19] S. Tasch, C. Brandstaetter, F. Meghdadi, G. Leising, G. Froyer, and L. Athouel, Adv. Mater. 9 (1997), 33. 
[20] J. Coraux, A.T. N’Diaye, C. Busse, and T. Michely, Nano Lett. 8 (2008), 565.

[21] A.T. N'Diaye, R. van Gastel, A.J. Martínez-Galera, J. Coraux, H. Hattab, D. Wall, F.J. Meyer zu Heringdorf, M. Horn von Hoegen, J.M. Gómez-Rodríguez, B. Poelsema, C. Busse, and T. Michely, New J. Phys. 11 (2009), 113056.

[22] E. Loginova, S. Nie, K. Thürmer, N.C. Bartelt, and K.F. McCarty, Phys. Rev. B 80 (2009), 085430.

[23] Z.H. Wang, K. Kanai, K. Iketaki, Y. Ouchi, and K. Seki, Thin Solid Films 516 (2008), 2711.

[24] Y.-H. Zhang, K.-G. Zhou, K.-F. Xie, J. Zeng, H.-L. Zhang, and Y. Peng, Nanotechnology 21 (2010), 065201.

[25] A.J. Fisher and P.E. Blöchl, Phys. Rev. Lett.70 (1993), 3263.

[26] OBForceFieldGhemical (2010).

<http://openbabel.org/wiki/OBForceFieldGhemical/>

[27] T. Hassinen and M. Peräkylä, J. Comput. Chem. 22 (2001), 1229.

[28] Avogadro version 1.0.0 (2010).

<http://avogadro.openmolecules.net/>

[29] S. Müllegger and A. Winkler, Surf. Sci. 600 (2006), 1290.

[30] K.N. Baker, A.V. Fratini, T. Resch, W.W. Adams, E.P. Socciand, and B.L. Farmer, Polymer 34 (1993), 1571.

[31] M. Henzler, Appl. Surf. Sci. 11-12 (1982), 450.

[32] M. Henzler, Surf. Sci. 19 (1970), 159.

[33] D.J. Gundlach, Nat. Mater. 6 (2007), 173. 


\section{Diffusion and submonolayer growth of 6P on $\operatorname{Ir}(111)$ and graphene}

Transparent, flexible, and conductive graphene sheets form an ideal substrate for the fabrication of organic light emitting diodes. In order to obtain an optimal final device, it is vitally important to understand the underlying nucleation and growth processes. In this chapter, the growth kinetics of 6P thin films on graphene and on $\operatorname{Ir}(111)$ has been investigated. Special attention has been paid to directed and concerted diffusion processes of 6P molecules on graphene grown on Ir(111). From the movement of large islands - formed by flat-lying molecules - across wrinkle-free graphene areas, the activation barrier for the diffusion of $6 P$-molecules along step edges of $6 P$-islands has been estimated to be about $0.55 \mathrm{eV}$. For the case of $6 P$ growth on $\operatorname{Ir}(111)$, ramified islands formed by upright standing molecules are found. Heteronucleation, in combination with particularities in the shape of possible smallest stable clusters, is identified as the root cause for the measured critical nucleus size of zero*.

*Published in IBM J. Res. Dev. 55 (2011), 15. 


\subsection{Introduction}

Devices based on small conjugated molecules are starting to enter the market but are limited in performance. These limitations arise at least partially from rough film morphology, misaligned grains, and the resulting grain boundaries $[1,2]$. Although, the understanding of the underlying growth processes is currently improving rapidly $[3,4]$, a widely accepted method for achieving the desired layer-by-layer growth is still to be found. The achieved layer-by-layer growth at low temperatures and the transition to a Stranski-Krastanov like growth at relatively high temperature is rooted in the interactions of $6 \mathrm{P}$ with both graphene and its supporting substrate $\operatorname{Ir}(111)$.

In this chapter, we describe in detail the nucleation, island evolution, and diffusion of $6 \mathrm{P}$ deposited on graphene flakes and on uncovered $\operatorname{Ir}(111)$ parts. The substrate induced changes of the diffusion behavior are discussed in detail, and models for the critical nucleus are proposed.

The combination of the organic semiconductor 6P, graphene, and the well established deposition technique of OMBE, will allow future flexible and low cost devices based on small conjugated molecules. 6P is well suited for the fabrication of blue [7], or color tunable [8] light emitting diodes. In addition, graphene is a flexible, highly conductive, and transparent electrode material $[9,10]$, ideally suited as a technological substrate for organic semiconductors $[11,12]$.

\subsection{Experimental}

Graphene flakes were grown on an $\operatorname{Ir}(111)$ surface [13]. The metal crystal was cleaned by high temperature exposure to Oxygen. Graphene was then formed by thermal decomposition of $\mathrm{C}_{2} \mathrm{H}_{4}$ on the hot $\operatorname{Ir}(111)$ surface [14]. Low boiling point impurities were carefully removed by thoroughly out-gassing the source material for several hours. 6P has been deposited onto the sample by means of a resistively heated Knudsen-cell evaporator designed for organic molecules. The substrate temperature was fixed at $240 \mathrm{~K}$ during deposition.

An Elmitec LEEM-III has been used to record images every second. The growth of graphene was followed in real-time using PEEM until sufficiently large flakes had formed on the surface. The molecular film formation has been followed using LEEM with a start voltage of $2 \mathrm{eV}$, well below the band gap of $3.1 \mathrm{eV}$ for $6 \mathrm{P}$. As a result, no beam induced degradation of the films was observed.

\subsection{P on graphene flakes}

The layer-by-layer growth of $6 \mathrm{P}$ on graphene flakes at $240 \mathrm{~K}$ proceeds in an interesting two-step mechanism. In a first step, islands composed of a relatively low density of flat lying molecules are formed on the graphene surface. With increasing coverage, these transform into a denser, full first monolayer, which now exhibits the structure of the (1-1-1) plane of bulk 6P, i.e. the layer then consists of interdigitating rows of molecules, rotated with respect to each other. This structure is described in detail in Chapter 6. However, the nucleation and growth behavior of the initial islands as well as their mobility require more specific attention. 


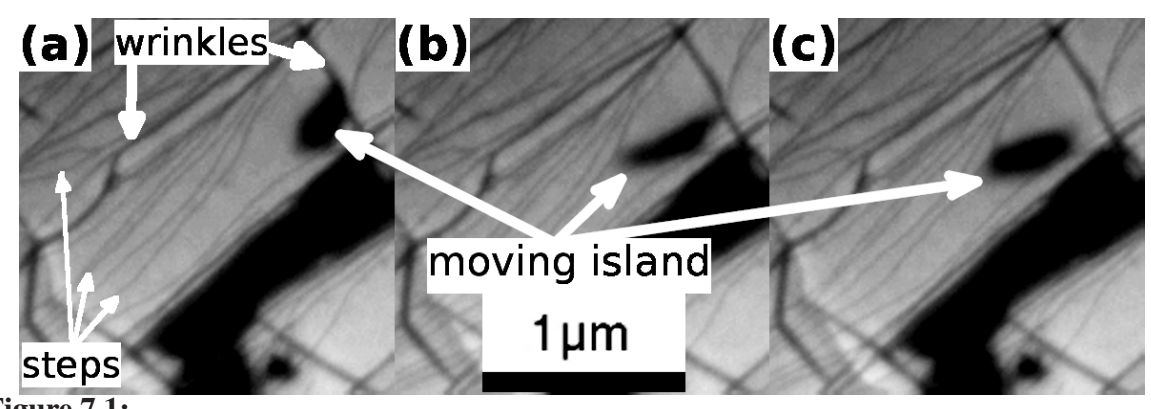

Figure 7.1:

Three consecutive LEEM images (one second between images) showing the mobility of the initial islands (dark patches) on graphene. A few graphene wrinkles (thick lines) and steps in the Ir(111) surface (thin lines) are marked. (a) A small 6P-island nucleated in the upper right corner next to a wrinkle and, (b) grows in size. (c) When the accumulated stress reaches a critical level, (middle image) the island elongates and detaches from the original site, to move to a location further away from the wrinkle. The entire process takes $2 \mathrm{~s}$.

Fig. 7.1 shows three LEEM images recorded within a time period of $2 \mathrm{~s}$. Fig. 7.1(a) shows the initial situation. An island has nucleated next to a wrinkle and has grown to a size of roughly $50,000 \mathrm{~nm}^{2}$. The aspect ratio of the island is 1:1.9. Fig. 7.1(b) shows the same island only one second later. It has elongated (aspect ratio 1:3; size: $55,000 \mathrm{~nm}^{2}$ ) and is about to detach from the wrinkle that acted as the nucleation site. When again one second later, the process is completed (Fig. 7.1(c)) the center of mass of the island has moved $280 \mathrm{~nm}$. The new position is clearly detached from the original nucleation site. The island shape returns to a more rounded outline (aspect ratio 1:2.3; size: $61,000 \mathrm{~nm}^{2}$ ) after the movement is completed. Note that the deposition continued during the sequence of images.

Careful analysis of many nucleation events reveals that the 6P islands start to grow in areas close to the wrinkles or next to wrinkle crossings. Highly curved graphene areas as the wrinkles themselves are unfavorable for the deposition of organic molecules [15]. Nevertheless, the islands nucleate in close proximity of the wrinkles. This can be explained by the following observation.

The wrinkles are formed during cool-down of graphene to room temperature to relieve compressive stress that originates from the different thermal expansion coefficients of graphene and Ir $[16,17]$. This stress relief is most effective close to the wrinkles leading to nearly strain free graphene in those regions. In areas further away from the wrinkles, compressive strain will remain. It is well know that for metallic systems, diffusion is faster on compressively strained areas [18-20]. Assuming the same behavior occurs for $6 \mathrm{P}$ on graphene, this leads to a higher diffusion rate further away from the wrinkles as compared to areas adjacent to the wrinkles. A higher diffusion rate will reduce the nucleation density on the strained part of the graphene and nucleation will preferentially occur close to the wrinkles.

The growth of the 6P island is characterized by an extremely high molecular mobility even at low temperatures. Subsequently, at low coverage, 6P islands can arrange in a way that minimizes free energy. Even though islands prefer to nucleate close to 
the wrinkles, they eventually change shape and diffuse to different locations on the graphene flake. At least two possible driving forces can be brought forward to account for this. First, a strain based argument will be given as a possible driving force for the island movement. A delicate balance between the size and shape of a $6 \mathrm{P}$ island and the total strain energy it holds, is one of the possible driving forces [21, 22]. As the island grows, tensile strain in the island will increase. This stress is a result of the lower density of the initial layer as compared to the later formed bulk structure and the mismatch between the phenyl ring spacing and the graphene ring spacing. Depending on the balance between stress and the step free energy, this can result in a shape transition of the island [23]. In our scenario, however, an additional pathway for relieving the strain exists. As mentioned above, metal supported graphene flakes are known to have areas that exhibit different levels of compressive strain. This compressive strain is lower next to wrinkles than further away from these defects. Depending on the local environment, a large island can thus become more stable in a more strained area which is found further away from the wrinkles. On compressively strained graphene, the molecules as well as the phenyl rings can relax into a closer packing and, therefore, reduce the tensile strain in the island.

A second argument is based on changes in the electronic structure. The electronic structure of graphene changes under the influence of the $\operatorname{Ir}(111)$ surface and the strain field created by the epitaxial mismatch [24]. Therefore, different properties can be expected in areas adjacent to the wrinkles and further away from them. Little is known about the precise electronic interaction between 6P and graphene. However, a slight charge transfer from 6P to the graphene can be expected based on theoretical work done for benzene on graphene [25]. From our experiments, we have no means to differentiate between the two scenarios. Most likely, both effects are active but with different strength.

During the transition shown in Fig. 7.1, an area of $55,000 \mathrm{~nm}^{2}$ has been cleared from 6P molecules. The size of the unit cell, which contains one molecule is $1.56 \mathrm{~nm}^{2}$ [5]. Assuming that the motion of $6 \mathrm{P}$ molecules proceeds along the edges of the island, we can estimate that at least 35,000 6P molecules needed to move from the back, to the front of the island. This corresponds to a distance of $400 \mathrm{~nm}$. Assuming the smallest possible step to be of the order of the graphene lattice constant (2.46 $\AA$ ), a total of $\nu=$ $2.9 \times 10^{7}$ hops is necessary to complete the process. This is an upper limit of hops for a directed movement of the molecules along the rim of the island. The activation barrier $E_{A}$ can then be calculated from

$$
\nu=\nu_{0} e^{-\frac{E_{A}}{k_{B} T}},
$$

where $\nu_{0}$ is the attempt frequency, $k_{B}$ the Boltzmann constant, and $T=240 \mathrm{~K}$ for the described situation. Using $\nu_{0}=1 \times 10^{13} \mathrm{~s}^{-1}$, an activation energy of $E_{A}=0.26 \mathrm{eV}$ is obtained. However for organic molecules, high attempt frequencies for desorption are reported experimentally [26-29] as well as theoretically [30-32]. For 6P in particular, an extreme value of $\nu_{0}=5.6 \times 10^{25} \mathrm{~s}^{-1}$ is reported for desorption experiments [28]. Furthermore, a recent study of $6 \mathrm{P}$ on modified mica shows that also the pre-exponential factor for surface diffusion is increased by four orders of magnitude [33]. In addition, 


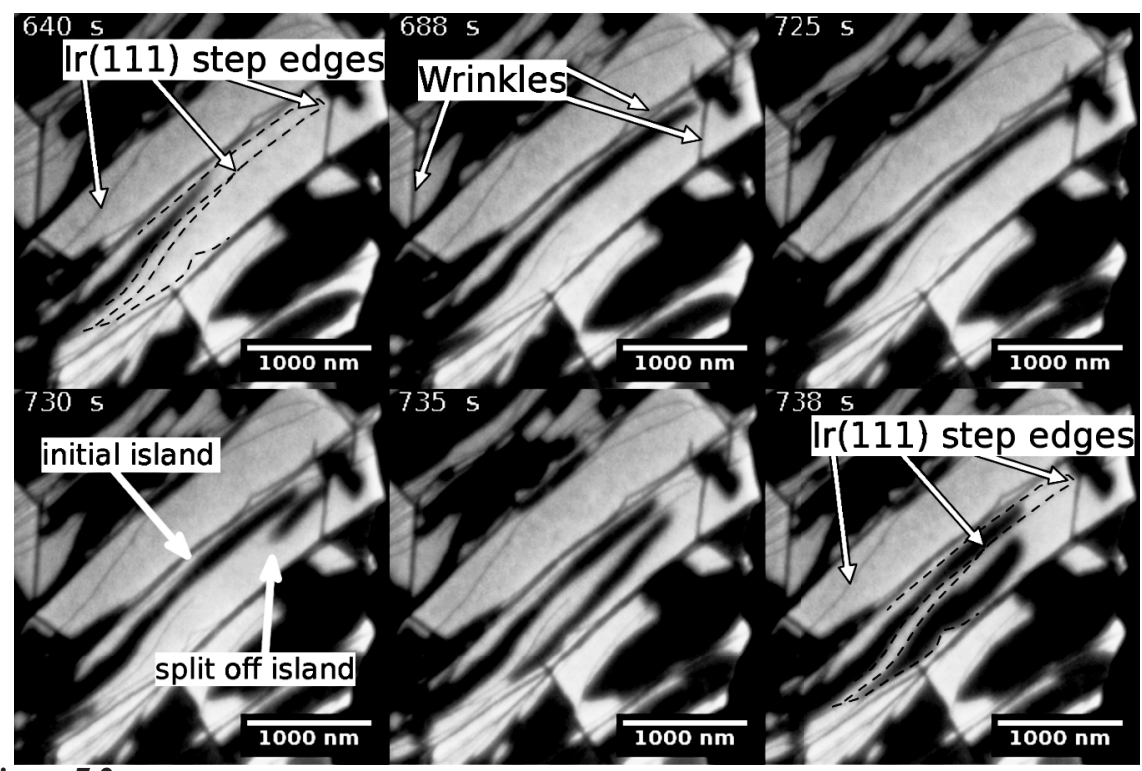

Figure 7.2:

Sequence of LEEM images recorded during the deposition of $6 \mathrm{P}$ on graphene at $240 \mathrm{~K}$. A narrow island (dark patches) which is confined between two steps slowly grows and finally fills the area enclosed by the steps (deposition time from $640 \mathrm{~s}$ to $688 \mathrm{~s}$ ). $32 \mathrm{~s}$ later, the island overcomes the step and spills out onto the adjacent graphene covered Ir(111) terrace (725 s of deposition). As the island on the new terrace grows in size (730s of deposition), it moves away from the low strain area next to wrinkle (735 s of 6P deposition). After reaching an area where the strain mismatch between the big $6 P$ island and graphene is reduced, it starts to fill the entire area by reducing its aspect ratio (738s of deposition). Please be aware of the non-uniform time step between the images. Wrinkles and Ir(111) step edges are marked by arrows and dashed lines to guide the eye.

Schunack et al. have observed an anomalous amount of long jumps in the surface diffusion of large organic molecules [34]. Both effects - changes in the apparent attempt frequency and heaped occurrence of long jumps might influence this value. Although, these activation barriers for diffusion are still lower limits - since we assumed a directed diffusion along the edge of the island - they roughly agree with other diffusion related values known for 6P. The binding energy of benzene on graphene has been calculated to be of the order of $0.16 \mathrm{eV}$ to $0.24 \mathrm{eV}$ [25]. As a first order approximation, $6 \mathrm{P}$ can be seen as a linear combination of 6 benzene rings. The binding energy of $6 \mathrm{P}$ on graphene is, therefore, expected to be between $1 \mathrm{eV}$ and $1.44 \mathrm{eV}$. The values for the diffusion energies reported here, despite sizable uncertainties, are substantially lower and, thus, do not conflict with the estimated binding energy. Processes as illustrated in Fig. 7.1 are always observed to be confined to an area enclosed by wrinkles. Often, the path of the islands is also confined by steps in the underlying Ir. The increased curvature of the graphene sheet at the position of the $\operatorname{Ir}(111)$ step poses a small but observable obstacle to the growth of the $6 \mathrm{P}$ islands. However, the effect is smaller than that due to the wrinkles. Fig. 7.2 depicts an island that is first overcoming a step edge, then splits 
in two, and finally moves across the graphene flake. Initially the island grows in size without crossing the surrounding steps until $720 \mathrm{~s}$ of $6 \mathrm{P}$ deposition. It then suddenly crosses the small end of the step enclosed area and spills out on the next graphene covered terrace. There, it quickly grows in size covering the area next to the wrinkle. As in the above situation, when a certain size is reached, it detaches from the wrinkle (island size: $117,400 \mathrm{~nm}^{2}$; aspect ratio: 1:10) and for this particular case also separates from the initial island. It continues to grow (final island size: $300,000 \mathrm{~nm}^{2}$; maximum aspect ratio: 1:13) while the center of mass moves $810 \mathrm{~nm}$ across the surface. After reaching an area where the mismatch between the 6P island and the strained graphene becomes small enough, also the shape transition is reversed leading to a lower aspect ratio of 1:6.

A detailed LEED study reveals the precise orientation of the molecules with respect to graphene lattice [5]. However, a relation of this data to the real space orientation of the molecules in each island is difficult if not impossible at all. From the way that the islands move on the surface, we can directly deduct the molecular orientation of the island in real space. Fig. 7.3 schematically shows a $6 \mathrm{P}$ island on a surface. Molecules are detaching from the left hand side and diffuse to the right hand side of the island (these are the edges parallel to the molecular axis, referred to as the parallel edge). This could be caused by a strain field induced by a wrinkle on the left side and the island exceeding a certain size. As the molecule diffuses around the island, no stable bond can be formed at the edge perpendicular to the long molecular axis (referred to as the perpendicular edge). Either the crystalline symmetry of the island has to be broken or the molecule is connected only by a single hydrogen bond. Both situations are unfavorable compared to incorporation at the parallel edge where at least six hydrogen bonds can be formed without breaking the symmetry of the crystalline structure. This is well known behavior of many anisotropic molecules. For thicker films, it will lead to the frequently observed needle or chain like growth of three-dimensional islands $[35,36]$. In these three-dimensional structures, the molecular axis is oriented perpendicular to the fiber axis. For flat lying 6P molecules on graphene, it leads to an anisotropic growth and directed movement of the islands. The preferred direction of island growth and movement is perpendicular to the long molecular axis of the $6 \mathrm{P}$ molecules forming the island. The frequency at which these processes occur and the speed of the actual transformation is a result of the high diffusion rate of individual 6P molecules in this configuration. As soon as any interdigitating, edge-on molecules are added to complete the first monolayer, the mobility of islands is reduced to a negligible value on the timescale of our experiment. For higher layers, no island mobility is observed. Compared to the first half layer, they constitute a more stable structure. This is also evident when comparing the initial open structure of the initial layer to the more bulk like arrangement of the thicker films [5].

\subsection{P on $\operatorname{Ir}(111)$}

Fig. 7.4(a) shows a graphene-free part of the iridium surface after the deposition of $6 \mathrm{P}$ has been stopped. A relatively large number of small irregularly shaped islands is visible. From $\mu$ LEED measurements, we obtain a typical intermolecular distance of $4.4 \AA$. This corresponds to the distance between the center molecule and the corner 


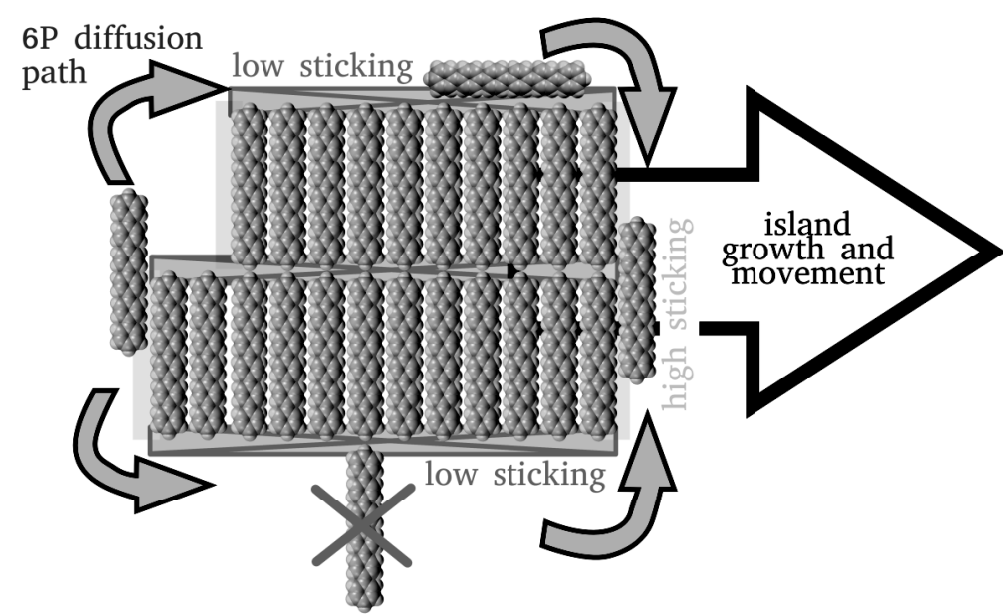

Figure 7.3:

Schematic $6 P$ islands outlining the possible diffusion paths and preferred incorporation sites. $6 P$ molecules detach from the left and move towards the right side of the island. The side facets are formed by the terminating hydrogen atoms of 6 , and posses a low sticking probability. Only the facets terminated by the long side of $6 P$ have a high sticking probability due to the large number of hydrogen bonds that can be formed there.

molecules in the (001)-plane of bulk 6P. The irregularly shaped islands on the $\operatorname{Ir}(111)$ surface are therefore formed from upright standing 6P molecules. Converting the coverage measured on the graphene flakes of 4.35 ML [5] of flat lying molecules to upright standing molecules, one expects $0.8 \mathrm{ML}$ of upright standing 6P. The coverage obtained from analyzing the LEEM images is only $0.5 \mathrm{ML}$. However, it is well known that a high step edge barrier which is effective during the growth of upright 6P islands, leads to a pronounced mound formation [3]. Assuming Poisson shaped mounds, the expected first layer coverage is $55 \%$ for a nominal film thickness of $0.8 \mathrm{ML}$ and in good agreement with the measured value.

In comparison to Fig. 7.1 and Fig. 7.2, it is evident that the nucleation density, $N_{s}$ $=7.2 \mu \mathrm{m}^{-2}$, on the Iridium surface is substantially higher than that for graphene, although no numbers can be given for the latter surface. From the capture zone distribution, we can calculate the size of the critical nucleus using the distribution function of the general Wigner-Seitz surmise as proposed in Ref. [37], i.e.,

$$
P_{\beta}(s)=a_{\beta} s^{\beta} e^{-b_{\beta} s^{2}},
$$

Here, $a_{\beta}^{\dagger}$ and $b_{\beta}{ }^{\ddagger}$ are constants given by the normalization and unit-mean conditions, respectively. The fluctuating variable, $s=A /\langle A\rangle$, is obtained from the areas of the Voronoi cells around the center of mass for each island. The only fit parameter $\beta=$ $i+2$ [38] allows to extract the critical nucleus size directly. In Fig. 7.4(b), the capture

\footnotetext{
${ }^{\dagger} a_{\beta}=2 \Gamma((\beta+2) / 2)^{\beta+1} / \Gamma((\beta+1) / 2)^{\beta+2}$, Here, the $\Gamma$ symbol refers to the gamma function in mathematics.

${ }^{\ddagger} b_{\beta}=[\Gamma((\beta+2) / 2) / \Gamma((\beta+1) / 2)]^{2}$
} 


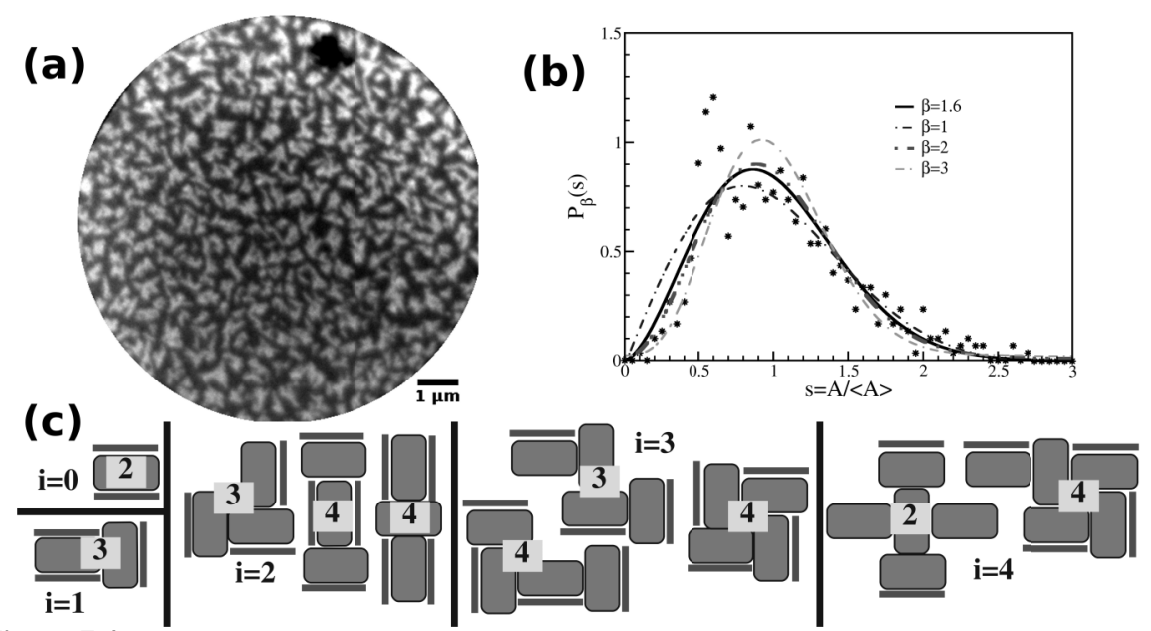

Figure 7.4:

$6 P$ growth on $\operatorname{Ir}(111)$. (a) LEEM image showing small islands formed by upright standing $6 P$ molecules on $\operatorname{Ir}(111)$. FOV $=10 \mu \mathrm{m}$. (b) Capture zone distribution for $6 P$ on $\operatorname{Ir}(111)$ grown at $240 \mathrm{~K}$. The best fit (solid line) to the data (stars) and distribution functions of the GWS for $\beta=1,2$, and 3 (line styles: dash-dot, dash-dot-dot and dash-dash-dot) are shown. (c) Possible configurations of the critical nucleus for $i=0$ to $i=4$. Upright standing $6 P$ molecules are represented by rectangles with an aspect ratio based on the Van der Waals dimensions of 6P. The $\pi$-system of the molecule is situated on the long sides of the rectangle. The central numbers on yellow background indicate the number of completely uncovered $\pi$-systems which are marked by red bars.

zone distribution for $6 \mathrm{P}$ islands on $\operatorname{Ir}(111)$ at $240 \mathrm{~K}$ is shown. The best fit $(\beta=1.6)$ and the curves for $\beta=1,2$, and 3 are shown. The critical nucleus size obtained in this way is zero, meaning a single molecule would be immobile on the surface and spontaneous nucleation proportional to the ad-molecule density should be observable.

To understand this result, we have to keep in mind that these calculations are strictly speaking only valid for homogeneous nucleation. A critical nucleus size of zero can be understood in the context of heterogeneous nucleation or defect nucleation $[39,40]$. $G$. Haas et al. explain the transition from $i=0$ to $i=3$, they observed in the $\mathrm{Pd} / \mathrm{MgO}$ system by the presence of defects, which act as traps and geometrical considerations that will make $i=3$ exceptionally stable [41]. The situation observed here is similar since both effects play a role in the growth of $6 \mathrm{P}$ on the $\operatorname{Ir}(111)$ surface. First, it is easy to understand that the $\operatorname{Ir}(111)$ surface is not clean. Carbon residues, maybe in the form of small graphene flakes, will be present all over the surface. This is also suggested by the fact that we observe upright standing molecules. One would expect a clean metal surface to have flat lying $6 \mathrm{P}$ molecules $[42,43]$. However, it has been shown that small amounts of carbon on the metal surface will change the orientation of the molecules and lead to films formed by upright standing molecules [44, 45]. Furthermore, it has been shown that defects such as dangling bonds can force molecules into an upright orientation, although, they would in principle energetically prefer to lie flat on the surface [46]. Second, certain assumptions can be made on the form of the critical nucleus. 
It is reasonable to assume that the molecules forming the nucleus are arranged in a configuration that is close to the bulk herringbone structure. Fig. 7.4(c) schematically shows $6 \mathrm{P}$ clusters starting from $i=0$ to $i=4$. The number of completely exposed $\pi$-systems is given for each configuration. It turns out that only $i=0$ and $i=4$ have a minimum number of two $\pi$-systems completely exposed. In general, one can assume that clusters with a small number of exposed $\pi$-systems are energetically favored. These numbers are also in reasonable good agreement with recent DFT calculations, which reveal a smallest stable cluster size of 4 for upright standing molecules [33]. As a result, for a sufficiently high trap binding energy, the formation of homogeneous nuclei with $i=1$ to $i=3$ is suppressed. Only at high enough temperatures, small clusters trapped at an impurity will break up and homogeneous nucleation with $i=4$ should become effective.

\subsection{Conclusions}

In this chapter, we have described the diffusion process of 6P molecules on graphene flakes and on iridium. The initial island formation on graphene is characterized by a high mobility of $6 \mathrm{P}$ molecules. This allows for strain driven shape and position changes of the initial submonolayer islands. A delicate interplay between intrinsic strain in the graphene flakes and strain that builds up with increasing island size is one of the possible driving force. However, electronic effects based on charge transfer between 6P and graphene, and, graphene and iridium are another possible cause. The growth of $6 \mathrm{P}$ on the $\operatorname{Ir}(111)$ surface yields ramified islands formed by upright standing molecules. Using capture zone scaling, we obtained a critical nucleus size of $i=0$. This result is interpreted as an indication for heterogeneous nucleation triggered by a high density of defects. The defects are most likely carbon clusters left over from the graphene formation or possibly small graphene flakes. As long as the trap binding energy of the defects for $6 \mathrm{P}$ is sufficiently high, a homogeneous nucleation with cluster size between 2 and 3 is suppressed. In addition, we present possible configurations for the critical nucleus for $i=0$ to $i=4$. Only the last one has a comparatively small number of fully exposed $\pi$-systems. We interpret this in terms of a reduced stability for the intermediate nuclei. In a scenario where nucleation is mostly defect driven, these cluster sizes are most likely suppressed due to dominant trapping at defect sites. 


\section{Bibliography}

[1] S. Verlaak, C. Rolin, and P. Heremans, J. Phys. Chem. B 111 (2007), 139.

[2] G. Horowitz and M.E. Hajlaoui, Adv. Mater. 12 (2000), 1046.

[3] G. Hlawacek, P. Puschnig, P. Frank, A. Winkler, C. Ambrosch-Draxl, and C. Teichert, Science 321 (2008), 108.

[4] X. Zhang, E. Barrena, D. Goswami, D.G. De Oteyza, C. Weis, and H. Dosch, Phys. Rev. Lett. 103 (2009), 136101.

[5] G. Hlawacek, F.S. Khokhar, R. van Gastel, B. Poelsema, and C. Teichert, Nano Lett. 11 (2011), 333.

[6] F.S. Khokhar, G. Hlawacek, R. van Gastel, C. Teichert, and B. Poelsema, accepted for publication in Surf. Sci. (2011).

[7] H. Yanagi and S. Okamoto, Appl. Phys. Lett. 71 (1997), 2563.

[8] S. Tasch, C. Brandstaetter, F. Meghdadi, G. Leising, G. Froyer, and L. Athouel, Adv. Mater. 9 (1997), 33.

[9] A.K. Geim and K.S. Novoselov, Nat. Mater. 6 (2007), 183.

[10] T.J. Echtermeyer, M.C. Lemme, M. Baus, B.N. Szafranek, A.K. Geim, and H. Kurz, IEEE Electron Device Lett. 29 (2008), 952.

[11] P. Lauffer, K.V. Emtsev, R. Graupner, T. Seyller, and L. Ley, Phys. Stat. Sol. B 245 (2008), 2064.

[12] Q. H. Wang and M. C. Hersam, Nat. Chem. 1 (2009), 206.

[13] J. Coraux, A. T. N'Diaye, C. Busse, and T. Michely, Nano Lett. 8 (2008), 565.

[14] R. van Gastel, A.T. N’Diaye, D. Wall, J. Coraux, C. Busse, N.M. Buckanie, F.-J. Meyer zu Heringdorf, M. Horn von Hoegen, T. Michely, and B. Poelsema, Appl. Phys. Lett. 95 (2009), 121901.

[15] F. Tournus and J.-C. Charlier, Phys. Rev. B, 71 (2005), 165421.

[16] A.T. N'Diaye, J. Coraux, T.N. Plasa, C. Busse, and T. Michely, New J. Phys. 10 (2008), 043033.

[17] A.T. N'Diaye, R. van Gastel, A.J. Martinez-Galera, J. Coraux, H. Hattab, D. Wall, F. Meyer zu Heringdorf, M. Horn von Hoegen, J.M. Gomez-Rodriguez, B. Poelsema, C. Busse, and T. Michely, New J. Phys. 11 (2009), 113056.

[18] H. Brune, K. Bromann, H. Röder, K. Kern, J. Jacobsen, P. Stoltze, K. Jacobsen, and J. Norskov, Phys. Rev. B 52 (1995), R14380.

[19] M. Schroeder and D.E. Wolf, Surf. Sci. 375 (1997), 129. 
[20] M. Gsell, P. Jakob, and D. Menzel, Science 280 (1998), 717.

[21] J. Tersoff and R.M. Tromp, Phys. Rev. Lett. 70 (1993), 2782.

[22] V.S. Stepanyuk, D.I. Bazhanov, W. Hergert, and J. Kirschner, Phys. Rev. B 63 (2001), 153406.

[23] H.J.W. Zandvliet and R. Van Gastel, Phys. Rev. Lett. 99 (2007), 136103.

[24] I. Pletikosic, M. Kralj, P. Pervan, R. Brako, J. Coraux, A.T. N'Diaye, C. Busse, and T. Michely, Phys. Rev. Lett. 102 (2009), 056808.

[25] Y.-H. Zhang, K.-G. Zhou, K.-F. Xie, J. Zeng, H.-L. Zhang, and Y. Peng, Nanotechnology 21 (2010), 065201.

[26] S.L. Tait, Z. Dohnalek, C.T. Campbell, and B.D. Kay, J. Chem. Phys. 122 (2005), 164708.

[27] P. Frank, T. Djuric, M. Koini, I. Salzmann, R. Rieger, K. Mullen, R. Resel, N. Koch, and A. Winkler, J. Phys. Chem. 114 (2010), 6650.

[28] S. Müllegger and A. Winkler, Surf. Sci. 600 (2006), 1290.

[29] K.R. Paserba and A.J. Gellman, Phys. Rev. Lett. 86 (2001), 4338.

[30] K.A. Fichthorn, K.E. Becker, and R.A. Miron, Catal. Today 123 (2007), 71.

[31] K.E. Becker and K.A. Fichthorn, J. Chem. Phys. 125 (2006), 184706.

[32] K.A. Fichthorn and R.A. Miron, Phys. Rev. Lett. 89 (2002), 196103.

[33] T. Potocar, S. Lorbek, D. Nabok, Q. Shen, L. Tumbek, G. Hlawacek, P. Puschnig, C. Ambrosch-Draxl, C. Teichert, and A. Winkler, Phys. Rev. B 83 (2011), 075423.

[34] M. Schunack, T.R. Linderoth, F. Rosei, E. Laegsgaard, I. Stensgaard, and F. Besenbacher, Phys. Rev. Lett. 88 (2002), 156102.

[35] H. Plank, R. Resel, H. Sitter, A. Andreev, N.S. Sariciftci, G. Hlawacek, C. Teichert, A. Thierry, and B. Lotz, Thin Solid Films 443 (2003), 108.

[36] F. Balzer, V.G. Bordo, A.C. Simonsen, and H.-G. Rubahn, Phys. Rev. B 67 (2003), 115408.

[37] A. Pimpinelli and T.L. Einstein, Phys. Rev. Lett. 99 (2007), 226102.

[38] A. Pimpinelli and T.L. Einstein, Phys. Rev. Lett. 104 (2010), 149602.

[39] G. Rosenfeld, R. Servaty, C. Teichert, B. Poelsema, and G. Comsa, Phys. Rev. Lett. 71 (1993), 895.

[40] D.D. Chambliss and K.E. Johnson, Phys. Rev. B 50 (1994), 5012. 
[41] G. Haas, A. Menck, H. Brune, J.V. Barth, J.A. Venables, and K. Kern, Phys. Rev. B 61 (2000), 11105.

[42] H. Oji, E. Ito, M. Furuta, K. Kajikawa, H. Ishii, Y. Ouchi, and K. Seki, J. Electron Spectrosc. Relat. Phenom. 101-103 (1999), 517.

[43] B. Winter, J. Ivanco, F.P. Netzer, and M.G. Ramsey, Thin Solid Films 433 (2003), 269.

[44] S. Müllegger and A Winkler, Surf. Sci. 574 (2005), 322.

[45] P. Frank, G. Hlawacek, O. Lengyel, A. Satka, C. Teichert, R. Resel, and A. Winkler, Surf. Sci. 601 (2007), 2152.

[46] L. Tsertseris and S.T. Pantelides, Appl. Phys. Lett. 87 (2005), 233109. 


\section{The influence of substrate temperature on growth of $6 \mathrm{P}$ thin films on graphene}

To complete our study of the behavior of 6P films on graphene, we have studied the growth at various substrate temperatures in real-time with LEEM. $\mu L E E D$ has been used to determine the structure of the different $6 P$ features formed on the surface. We observe the nucleation and growth of a wetting layer in the initial stages of growth. Graphene defects - wrinkles - are found to be preferential sites for the nucleation of the wetting layer and $6 P$ needles that grow on top of the wetting layer in the later stages of deposition. The molecular structure of the wetting layer and needles is found to be comparable. As a result, only a limited number of growth directions are observed for the needles. The formation of ramified structures formed by upright standing $6 P$ molecules is observed on the bare Ir(111) surface at $320 \mathrm{~K}$ and $352 \mathrm{~K}$. At $405 \mathrm{~K}$, the formation of a continuous layer of upright standing molecules growing in a step flow like manner is observed on $\operatorname{Ir}(111)^{*}$.

*Surf. Sci. (2011), in press 


\subsection{Introduction}

In recent years, the growth of organic semiconductors on solid substrates has received significant attention for both scientific and technological reasons. One such organic semiconductor is $6 \mathrm{P}$, a rigid rod-like conjugated molecule. Thin film growth of $6 \mathrm{P}$ molecules has been investigated intensely due to the unique optical and electronic properties of the molecule. These properties are found to be subject to substrate anisotropy and also depend on the arrangement of the molecules in a thin film $[1,2]$. The molecular orientation can be controlled by using appropriate substrates from lying [3] to upright standing [4]. In-depth knowledge of the growth behavior as a function of temperature is a key to control the thin film structure and exploiting its full technological potential [5]. In several recent publications, it has been shown how the growth parameters can be used to tailor the morphology of 6P thin films on different substrates [6-8]. In this paper, we investigate the growth and structure of $6 \mathrm{P}$ molecules at different surface temperatures on epitaxially grown graphene sheets supported by an $\operatorname{Ir}(111)$ surface. The layers and needles that form on graphene as well as the ramified structures that grow on $\operatorname{Ir}(111)$ are studied as a function of substrate temperature. The role of defects in the graphene sheets is also analyzed using LEEM and PEEM. $\mu$ LEED is used to locally obtain structural information [9].

\subsection{Experimental}

The experiments are carried out in an Elmitec LEEM III apparatus of Bauer's design [10] with a base pressure of less than $1 \times 10^{-10}$ mbar. A $1.4 \mu \mathrm{m}$ field-limiting aperture has been utilized to collect local structural information from features of interest. An $\operatorname{Ir}(111)$ substrate is atomically cleaned by exposing to low pressures of $\mathrm{O}_{2}$ at elevated temperature. Graphene films are then prepared by CVD of $\mathrm{C}_{2} \mathrm{H}_{4}$ on the $\operatorname{Ir}(111)$ surface at a temperature of $875 \mathrm{~K}$ [11]. The growth of the graphene flakes is followed in-situ using PEEM until sufficiently large graphene flakes have formed on the $\operatorname{Ir}(111)$ surface. A LEEM image of such a flake is shown in Fig. 8.1(a). Substrate steps (thin lines, indicated by white arrows) are still visible in Fig. 8.1(a) as the graphene flake follows the topographic contours of the underlying substrate. A network of straight linear features (indicated by black arrows), appearing much darker and wider than the steps, is also visible on the graphene. These linear features are wrinkles in the graphene sheet that result from elastic relaxations that occur when the sample is cooled from the graphene growth temperature to the 6P deposition temperature. The wrinkles extend about $3 \mathrm{~nm}$ from the surface and are a few nanometers in width [12]. Commercially available 6P molecules in powder form are deposited by OMBE from a Knudsen-cell type evaporator that is held at a temperature of $553 \mathrm{~K}$ for all described experiments. From previous experiments, it was calibrated to yield an average growth rate of $6.3 \times 10^{-4} 6 \mathrm{P} /\left(\mathrm{nm}^{2} \mathrm{~s}\right)$. This corresponds to a growth rate of $2.7 \mathrm{ML} / \mathrm{h}$ of flat lying $6 \mathrm{P}$ molecules [13]. We use the term monolayer for a closed layer of molecules having the mentioned structure. The number of 6P molecules per surface atom varies between $0.0156 \mathrm{P}(1 \overline{11}) /$ graphene and $0.286 \mathrm{P}(100) / \mathrm{Ir}(111)$, consequently only deposition times and molecular densities are given. The sample temperature during deposition of $6 \mathrm{P}$ has been varied between $320 \mathrm{~K}$ and $405 \mathrm{~K}$. In what follows, we will refer to this as the de- 

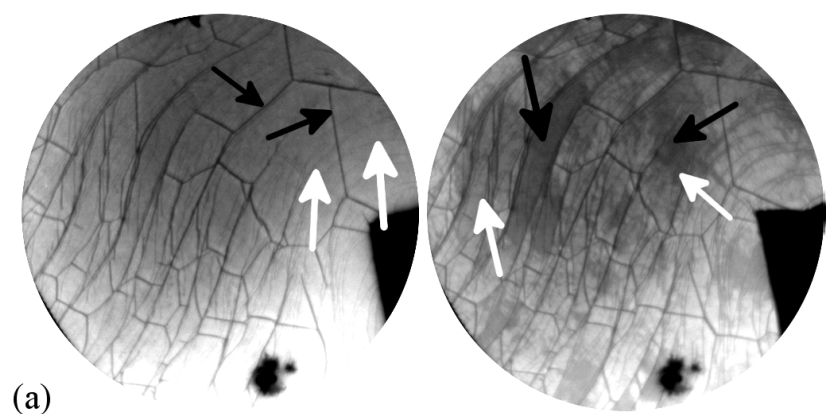

(a)

Figure 8.1:
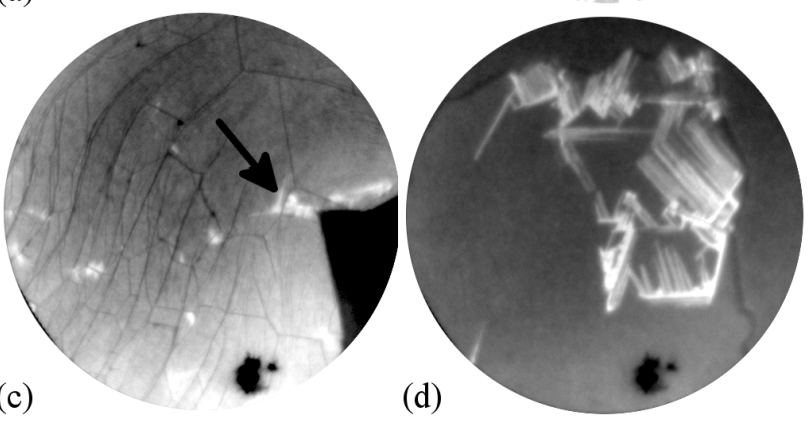

LEEM images acquired at a temperature of $320 \mathrm{~K}$ with an electron energy of $2.7 \mathrm{eV}$ for $(\mathrm{a})-(\mathrm{c})$ and $3.7 \mathrm{eV}$ for $(d)$. FoV is $6 \mu \mathrm{m}$ for $(a)-(c)$ and $4 \mu \mathrm{m}$ for $(d)$. Times indicated are measured with respect to the start of $6 P$ deposition. $(\mathbf{a}, \mathbf{t}=\mathbf{0} \mathbf{s})$ A single graphene flake on the $\operatorname{Ir}(111)$ surface is imaged prior to exposure to 6P. Graphene wrinkles (indicated with black arrows) and the faint contours of Ir(111) surface steps (indicated with white arrows) are visible on the single layer graphene flake. ( $\left.\mathbf{b}, \mathbf{t}=\mathbf{8 1 3} \mathrm{s}, \mathbf{0 . 5 2} \mathbf{6 P} / \mathbf{n m}^{2}\right)$ The graphene flake is covered by a wetting layer of 6P. The two additional grey levels correspond to the initial layer formed by flat lying face-on molecules only (white arrows), and the final wetting layer with a face-on/edge-on, (111) like structure (black arrows). The nucleation of this film happens next to the wrinkles. $(\mathbf{c}, \mathbf{t}=\mathbf{1 2 6 8} \mathbf{s}$, $0.806 \mathrm{P} / \mathbf{n m}^{2}$ ) Bright crystallites occur on top of the wetting layer next to the wrinkles (black arrow). $\left(\mathbf{d}, \mathbf{t}=2149 \mathrm{~s}, \mathbf{1 . 3 6} 6 \mathrm{P} / \mathbf{n m}^{2}\right)$ Parallel needles continue to grow with ongoing deposition. The dark area in the lower part of the images is a defect in the channel plate.

position temperature. These deposition temperatures are precise relative to each other. However, thermal effects in the sample holder might lead to a small but unknown offset of all temperatures given throughout the text.

\subsection{Results and discussion}

\subsubsection{Deposition of $6 \mathrm{P}$ at $320 \mathrm{~K}$}

A sequence of bright field LEEM images acquired during the deposition of $6 \mathrm{P}$ molecules is shown in Fig. 8.1. Fig. 8.1(a) shows the pristine graphene surface with graphene wrinkles (thick straight lines) and steps in the underlying $\operatorname{Ir}(111)$ surface (thin curved lines). For a detailed discussion of the morphology of graphene flakes on $\operatorname{Ir}(111)$, the reader is referred to $[11,12,14]$. With the deposition of $6 \mathrm{P}$ molecules, 
the intensity of reflected electrons from the graphene decreases, indicating the presence of a diluted phase of $6 \mathrm{P}$ molecules on the surface. After $727 \mathrm{~s}\left(0.466 \mathrm{P} / \mathrm{nm}^{2}\right)$ of deposition, nucleation of $6 \mathrm{P}$ domains takes place next to the wrinkles. The domains are mobile and move over the graphene surface [15]. After $813 \mathrm{~s}\left(0.526 \mathrm{P} / \mathrm{nm}^{2}\right)$ of deposition, the intensity that is measured on $6 \mathrm{P}$ domains reduces even further (indicated by black arrows in Fig. 8.1(b)). The dark 6P domains grow to form a complete monolayer after $948 \mathrm{~s}\left(0.606 \mathrm{P} / \mathrm{nm}^{2}\right)$ of $6 \mathrm{P}$ deposition. For the next $130 \mathrm{~s}$, no new features or significant contrast changes are observed. After this, bright $6 \mathrm{P}$ crystallites can be observed. These crystallites also nucleate next to the wrinkles as indicated by the black arrow in Fig. 8.1(c). In contrast to the initial islands, these crystals are immobile. With continued deposition, they elongate resulting in a fiber like morphology. Fig. 8.1(d) shows a LEEM image after stopping $6 \mathrm{P}$ growth at $2149 \mathrm{~s}\left(1.366 \mathrm{P} / \mathrm{nm}^{2}\right)$. The graphene surface is covered by a $6 \mathrm{P}$ wetting layer of monolayer thickness and long fiber-like structures, which nucleated either from defects in the wetting layer caused by the wrinkles, or from other needles.

Fig. 8.2(a) shows a $\mu$ LEED pattern that is obtained from an area without needles which is only covered by the monolayer thick wetting layer. The $\mu$ LEED pattern consists of the specular reflection surrounded by several rings of LEED spots. It reveals an ordered molecular structure. Within the $1.4 \mu \mathrm{m}$ aperture that we used to obtain the $\mu$ LEED pattern, several different rotational domains are present. Careful analysis of the $\mu$ LEED pattern also shows that the 6P molecules are arranged in two different ways, in other words, there are two different phases present. The unit cells are highlighted with solid and dotted lines. The length of the unit cell vectors, highlighted with dashed lines, are $5.2 \AA$ and $27.8 \AA$ at an angle $\beta$ of $72^{\circ}$. Here, $\beta$ is the angle between the two lattice vectors. The angle $\Theta$ between the long axis of the $6 \mathrm{P}$ unit cell and the graphene unit cell vector is $79^{\circ}$. The dimensions of the unit cell vectors, highlighted with solid lines, are $8.3 \AA$ and $27.8 \AA$ at an angle $\beta$ of $70^{\circ}$. Taking into account distortions in the LEED pattern, these numbers are accurate within $5 \%$. In accordance with the results obtained at $240 \mathrm{~K}$ [13], we assume that the first small unit cell contains one molecule in a face-on configuration (Fig. 8.2(c)) while the second larger unit cell contains two molecules which are assembled in a face-on - edge-on arrangement (Fig. 8.2(d)). The latter arrangement is similar to the one found in the surface unit cell of the bulk $6 \mathrm{P}(1 \overline{11})$ plane [17]. Also the size of the unit cell is similar to the bulk surface unit cell. However, the underlying substrate does not allow the film to relax completely. This results in a larger spacing along the long molecular axis. We obtain the following matrix notations for the unit cell vectors of the adsorbate lattice in terms of the substrate lattice vectors $\left(\mathrm{a}=\mathrm{b}=2.46 \AA\right.$ and $\left.\alpha=120^{\circ}\right)$ : for the inital layer $\left(\begin{array}{cc}8.6 & 12.8 \\ -1.3 & 1.2\end{array}\right)$ while the final bulk like layer has a matrix notation of $\left(\begin{array}{cc}8.6 & 12.8 \\ -1.9 & 2.0\end{array}\right)$. These latter values show a good match with structural data $\left(\left(\begin{array}{cc}8.7 & 13.0 \\ -1.7 & 1.9\end{array}\right)\right.$ for the final layer) obtained at a much lower temperature of $240 \mathrm{~K}$ [13]. The fact that this relationship between the 6P layer and graphene does not change over a temperature range of at least $80 \mathrm{~K}$ is a strong hint towards a fixed relationship between the two. Keeping in mind the accuracy of our initial measurements, we, therefore, interpret this as a coincidence type II quasiepitaxial relationship [16]. In fact, a $5 \times 10$ superstructure describes the layer more accurately. Taking into account the superstructure, we arrive at the following matrix notations for the initial $\left(\begin{array}{cc}43 & 64 \\ -13 & 12\end{array}\right)$ 


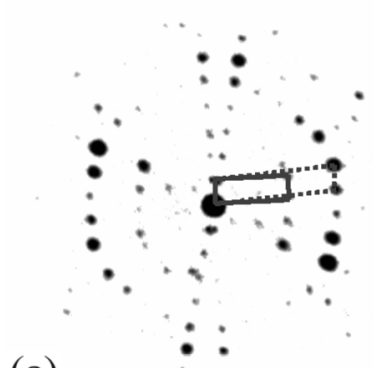

(a)

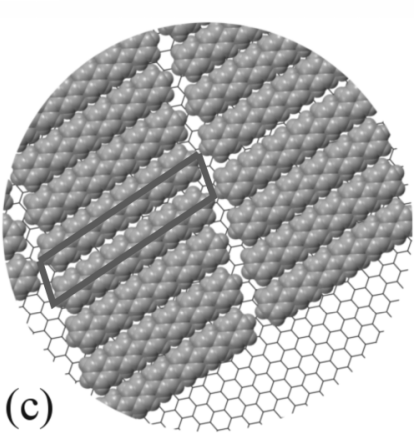

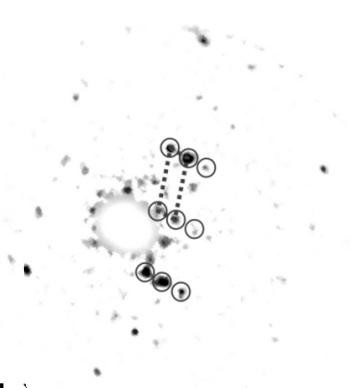

(b)

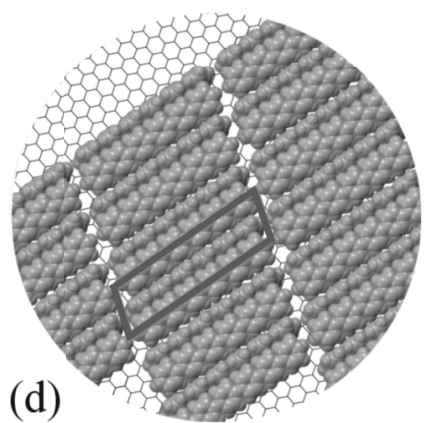

Figure 8.2:

(a) $\mu L E E D$ pattern measured from graphene covered with one monolayer of $6 P$ at an electron energy of $14 \mathrm{eV}$. The specular reflection and other LEED spots associated with various rotational domains of the ordered $6 P$ structure are visible. (b) $\mu L E E D$ pattern measured from a graphene area covered by needles at an electron energy of $21 \mathrm{eV}$. The LEED spots are marked with red circles to guide the eyes. (c) Molecular arrangement corresponding to the dashed unit cell in (a). The unit cell contains one face-on molecule. (d) Sketch of the molecular arrangement corresponding to the solid unit cell in (a). Two molecules per unit cell in an alternating face-on - edge-on configuration are found here. The molecular arrangement in the needles $(b)$ is similar to this second denser phase present in the wetting layer.

and the final monolayer thick wetting layer $\left(\begin{array}{cc}43 & 64 \\ -19 & 20\end{array}\right)$. This also better reflects the fact that in the superstructure the flexible molecules are free to relax their orientation and position in the superstructure by small amounts.

A typical $\mu$ LEED pattern taken from needles is shown in Fig. 8.2(b). It consists of LEED spots from a single domain and, thus, reveals an ordered molecular structure. The dimensions of the unit cell vectors are $9.5 \AA$ and $26.9 \AA$ at an angle $\beta$ of $69^{\circ}$. The molecular arrangement is similar to the second denser phase found in the wetting layer (fig. 8.2(d)). Again these values are very similar to the size of the $6 \mathrm{P}(1 \overline{11})$ surface unit cell and the size of the bigger unit cell found in the wetting layer. However, the three dimensional shape of the fiber crystallites allows the unit cell to relax towards the bulk value.

The growth of $6 \mathrm{P}$ on graphene at $320 \mathrm{~K}$ can be summarized by the following four steps. (1) An initial layer of only flat lying molecules is formed on the graphene surface. This layer nucleates next to the wrinkles. (2) When a critical coverage is reached, 
the initial layer transforms into a bulk like layer (Fig. 8.1(b)). The molecules obtain a flat face-on - edge-on configuration similar to the $6 \mathrm{P}(1 \overline{11})$ plane. (3) 6P fibers nucleate on top of the monolayer thick wetting layer (Fig. 8.1(c)). This nucleation occurs next to the wrinkles. (4) Parallel bundles of needles grow away from the wrinkles (Fig. 8.1(d)). The needles have the same $(1 \overline{11})$ orientation as the underlying wetting layer. The azimuthal orientation of the long needle axis is roughly perpendicular to the azimuthal orientation of the long unit cell axis and the long molecular axis. Nearly all nucleation
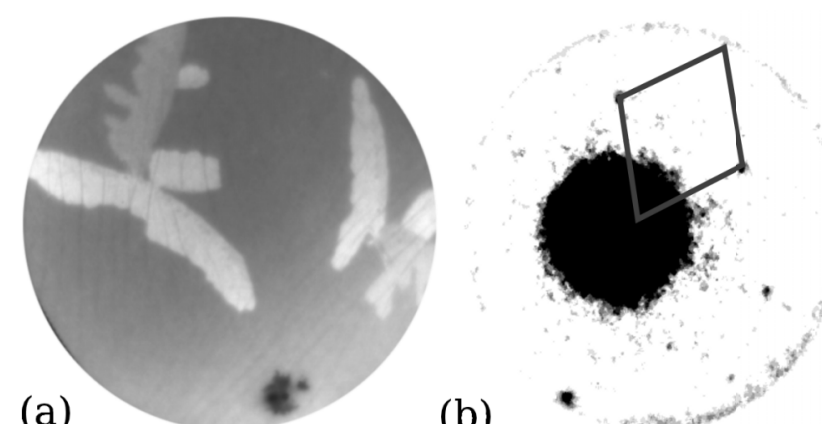

(a)

(b)

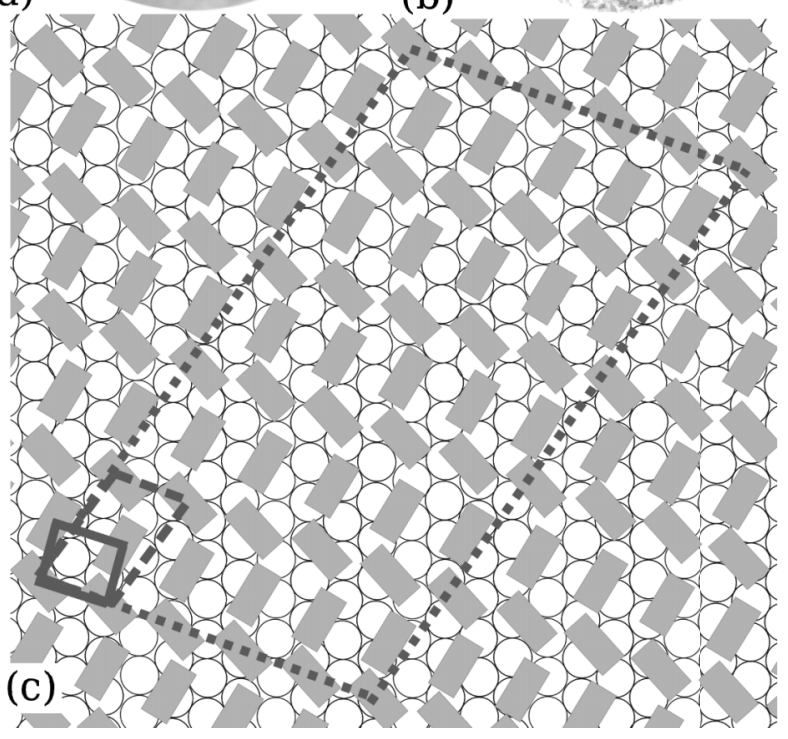

Figure 8.3:

(a) LEEM image of irregularly shaped structures of 6P grown on the Ir(111) surface. The Ir(111) surface appears dark and the ramified $6 P$ islands show different shades of grey. (FoV: $15 \mu \mathrm{m}$, electron energy: $3.5 \mathrm{eV}$, deposition temperature: $320 \mathrm{~K}$ ) (b) $\mu L E E D$ pattern obtained from one of the islands at an electron energy of $19.4 \mathrm{eV}$. The nearest neighbor cell is highlighted by red lines. (c) The structural model proposed from the $\mu L E E D$ pattern shown in (b). The molecules are arranged in up-right standing orientation on Ir(111). Nearest neighbor cell, unit cell, and the $5 \times 5$ superstructure are indicated by red lines (solid, dashed, and dotted, respectively).

events are occurring next to the wrinkles. The change in curvature of the graphene next to the wrinkle, strain in the adsorbed $6 \mathrm{P}$ islands, and the high mobility are responsi- 
ble for the preferred nucleation of the wetting layer next to wrinkles and the observed large domain size which is in the $\mu \mathrm{m}$ range. The preferred nucleation, mobility, and formation of the initial wetting layer of $6 \mathrm{P}$ on graphene is discussed in detail, elsewhere $[15,13]$. The wrinkles - by creating a large network of one-dimensional defects in the $6 \mathrm{P}$ wetting layer - are responsible for the preferred nucleation of the needles next to them. Although, the graphene flakes cover extended areas of the $\operatorname{Ir}(111)$ sur-
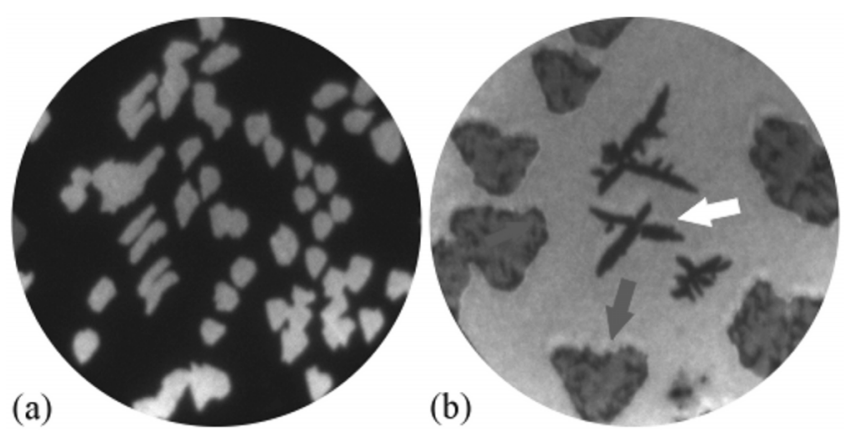

Figure 8.4:

(b)

$(\mathbf{a}, \mathbf{t}=\mathbf{2 0 5} \mathbf{s}) 100 \mu \mathrm{m}$ FoV PEEM image of Ir(111) covered with graphene flakes. The Ir(111) surface appears dark since its work function $(5.76 \mathrm{eV})$ is higher than the photon energy $(4.9 \mathrm{eV})$. $(\mathbf{b}, \mathbf{t}=\mathbf{2 1 4 9} \mathbf{s}) 50 \mu \mathrm{m}$ FoV PEEM image acquired after $6 P$ deposition at a temperature of $320 \mathrm{~K}$. The $6 P$ structures, graphene flakes, and ramified islands on Ir(111) are marked with red, green, and, white arrows, respectively. Times indicated are measured with respect to the start of $\mathrm{C}_{2} \mathrm{H}_{4}$ and $6 P$ deposition, respectively.

face, they still do not cover the entire surface. The remaining bare $\operatorname{Ir}(111)$ surface areas are inspected after stopping the deposition of $6 \mathrm{P}$ molecules $\left(1.366 \mathrm{P} / \mathrm{nm}^{2}\right)$. LEEM images show the presence of irregularly shaped 6P structures as presented in Fig. 8.3(a). A $\mu$ LEED measurement obtained from a branch of one of the irregularly shaped structures is shown in Fig. 8.3(b). The $\mu$ LEED pattern reveals that $6 \mathrm{P}$ molecules form an ordered structure on the $\operatorname{Ir}(111)$ surface. The dimensions of the nearest neighbor cell vectors are $5.0 \AA$ by $5.0 \AA$ at an angle $\beta$ of $108^{\circ}$. The size of this nearest neighbor cell implies that in these irregularly shaped structures the long axis of the molecules is roughly perpendicular to the surface. However, the cell vectors given above are the nearest neighbor distances and not the real unit cell vectors. This is a consequence of the molecular form factors for the two differently rotated upright standing molecules being nearly identical. The unit cell vectors are: $5.0 \AA$ by $9.1 \AA$ at an angle $\beta$ of $105^{\circ}$ and $\Theta=25^{\circ}\left(\left(\begin{array}{rr}3.8 & 1.6 \\ -0.4 & 1.6\end{array}\right)\right)$. Considering the above mentioned measurement precision and the fact that some of the molecules will shift slightly to reach a more favorable position, a $5 \times 5$ superstructure with a matrix notation of $\left(\begin{array}{rr}19 & 8 \\ -2 & 8\end{array}\right)$ (a coincidence type II quasiepitaxial relationship [16]) describes the situation more accurately. This can be seen in fig. 8.3(c) where some of the molecules would need to be shifted only slightly by fractions of an Ångstrom to reach a well coordinated site. The 6P molecules are arranged in a similar (up-right standing) fashion as in the (100) plane of the 6P bulk crystal. Different 6P islands or arms of them can have different azimuthal crystallographic orientations. This has been made visible in Fig. 8.3(a) by using a slightly off 
normal incident of the electron beam. As a result, different crystallographic orientations show different intensities similar to a dark field image. $\mu$ LEED patterns recorded away from the irregular structures consist only of $\operatorname{Ir}(111)$ spots and a dominant diffuse background. The latter is attributed to an unordered two-dimensional gas phase layer of $6 \mathrm{P}$ present on the surface of the Ir.

It is well known that on clean metal surfaces para-n-phenyl oligomers prefer a lying configuration [18-20]. However, small amounts of surfactants will lead to an upright standing configuration of the molecules [20, 19, 21, 22]. Therefore, it is reasonable to assume that carbon residues of the graphene growth are causing the appearance of these irregularly shaped structures on $\operatorname{Ir}(111)$. PEEM relies on photo-emitted electrons
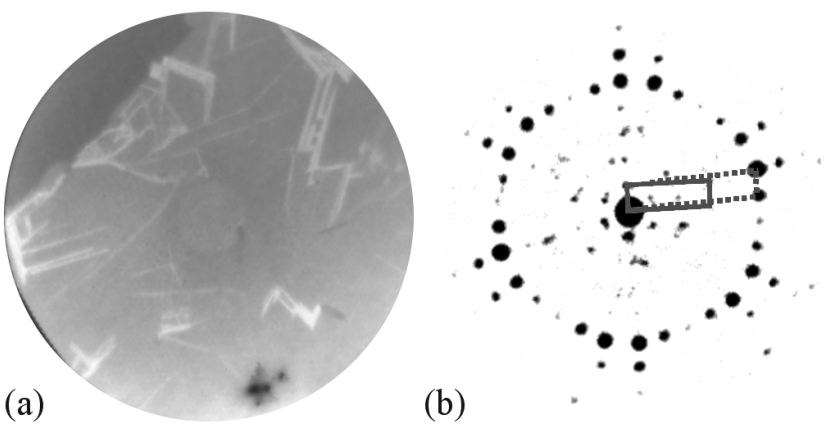

(a)

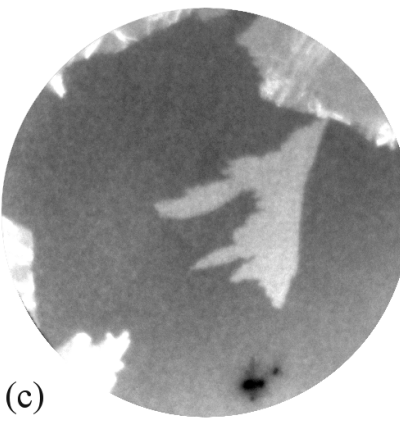

(b)

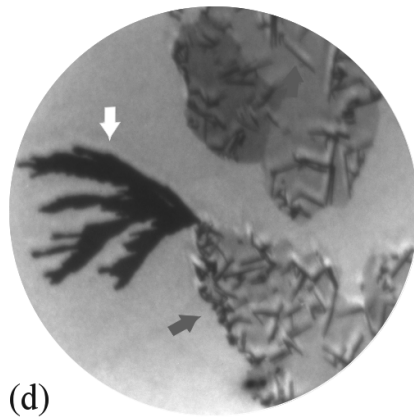

Figure 8.5:

$\left(\mathbf{a}, \mathbf{t}=2130 \mathrm{~s}, 1.356 \mathrm{P} / \mathbf{n m}^{2}\right) 10 \mu \mathrm{m}$ Fo V LEEM image acquired at an electron energy of $2.7 \mathrm{eV}$ and surface temperature of $352 \mathrm{~K}$. A single graphene flake on the $\operatorname{Ir}(111)$ surface is imaged after stopping deposition of $6 P$. The edge of the graphene flake is visible in the upper left part. The graphene flake is covered with $6 P$ needles of different orientation. (b) $\mu L E E D$ pattern measured from graphene covered by the wetting layer at an electron energy of $19.3 \mathrm{eV}$. (c) $20 \mu \mathrm{m} \mathrm{FoV}$ LEEM images acquired at an electron energy of $2.7 \mathrm{eV}$ and a temperature of $352 \mathrm{~K}$. The $\operatorname{Ir}(111)$ surface with an irregular shaped island and three graphene flakes covered with $6 P$ is visible. The $6 P$ island on the Ir(111) surface is connected to the graphene flake. (d) $50 \mu \mathrm{m}$ FoV PEEM image acquired after stopping the 6P deposition. $6 P$ needles, graphene flakes, and $6 P$ islands on Ir are present and marked by red, green, and white arrows, respectively $(352 \mathrm{~K})$.

and, therefore, depends on changes in the work function of a sample to create image contrast. The clean $\operatorname{Ir}(111)$ surface appears dark since its work function (5.76 eV [23]) is higher than the photon energy $(4.9 \mathrm{eV})$ whereas the graphene $(4.8 \mathrm{eV}-4.9 \mathrm{eV}$ [24]) 
flakes appear bright (Fig. 8.4(a)). However after deposition of 6P, the $\operatorname{Ir}(111)$ surface appears brighter than graphene (Fig. 8.4(b)). The change in contrast is suggestive of a surface work function variation caused by $6 \mathrm{P}$ adsorption and the formation of an interface dipole - both on $\operatorname{Ir}(111)$ and graphene. The 6P needles grown on graphene (indicated by a red arrow) appear darker than the $6 \mathrm{P}$ wetting layer on the graphene (Fig. 8.4(b). A white arrow is indicating the irregularly shaped structures on the $\operatorname{Ir}(111)$ surface, which gives a relatively darker contrast. The $6 \mathrm{P}$ covered graphene flakes appear darker than $\operatorname{Ir}(111)$ and have lighter shade of grey than the 6P needles. Therefore, the resulting order in brightness (from low to high) of the materials roughly grouped by work function is: $\operatorname{Ir}(111)$ and upright standing 6P islands on $\operatorname{Ir}(111)$ (both higher or similar to the photon energy), $6 \mathrm{P}(1 \overline{11})$-needles, $6 \mathrm{P}(1 \overline{11})$ wetting layer on graphene, disordered $6 \mathrm{P}$ on $\operatorname{Ir}(111)$. The non-emitting $6 \mathrm{P}$ needles are, therefore, only visible because they sit on a brighter background. This is similar to the contrast mechanism observed for the case of $6 \mathrm{P} / \mathrm{Cu}(110) 2 \times 1-\mathrm{O}$ [25].

\subsubsection{Deposition of $6 \mathrm{P}$ at $352 \mathrm{~K}$}

Increasing the deposition temperature to $352 \mathrm{~K}$ leads to no principle changes in the film formation process. After the initial two-step formation of a wetting layer by nucleation of domains near the wrinkles - the growth of parallel needles sets in. Again the needles nucleate either near the wrinkles or from existing needles creating comb like structures (Fig. 8.5(a)). As expected, higher deposition temperatures and the resulting enhanced mobility of 6P leads to fewer but longer needles [26].

A typical $\mu$ LEED pattern measured from the graphene surface covered by the wetting layer is shown in Fig. 8.5(b). The $\mu$ LEED pattern consists of the specular beam reflection surrounded by several rings of LEED spots. This $\mu$ LEED pattern is similar to the one obtained at $320 \mathrm{~K}$, presented in Fig. 8.2(a). The structure of the wetting layer at this elevated temperature is identical to the one that was already found for the growth at $320 \mathrm{~K}$. Due to the small signal, no reliable structural information could be obtained from the needles. However, taking into account the similarities in the wetting layer and the comparable morphology, one can conclude their structure is similar to the structure at $320 \mathrm{~K}$, presented in Fig. 8.2(d).

Post-deposition $\left(2130 \mathrm{~s}, 1.356 \mathrm{P} / \mathrm{nm}^{2}\right)$, LEEM imaging of the $\operatorname{Ir}(111)$ surface reveals the presence of branched 6P structures (Fig. 8.5(c)). All 6P structures on Iridium nucleate at the edges of the graphene flakes. The increased mobility of $6 \mathrm{P}$ on $\operatorname{Ir}(111)$ at this high temperature requires the stable graphene flakes for nucleation. Once formed, they act as sinks for all 6P diffusing on the $\operatorname{Ir}(111)$ surface. A similar structure of upright molecules as observed for the other deposition temperatures is proposed.

A PEEM image acquired after stopping the deposition of 6P is shown in Fig. 8.5(d). The 6P needles on the graphene flake (indicated with a red arrow) appear darker than the $6 \mathrm{P}$ wetting layer in the same way as described above. A white arrow marks the irregular and branched structures on the $\operatorname{Ir}(111)$ surface. Again, they show a darker contrast than the surrounding surface. The $6 \mathrm{P}$ wetting layer on the graphene flakes itself shows an intermediate grey level.

Post deposition annealing of the film leads to a decay of the structures. From deposition temperature to $381 \mathrm{~K}, 6 \mathrm{P}$ structures on graphene and $\operatorname{Ir}(111)$ remain intact and 

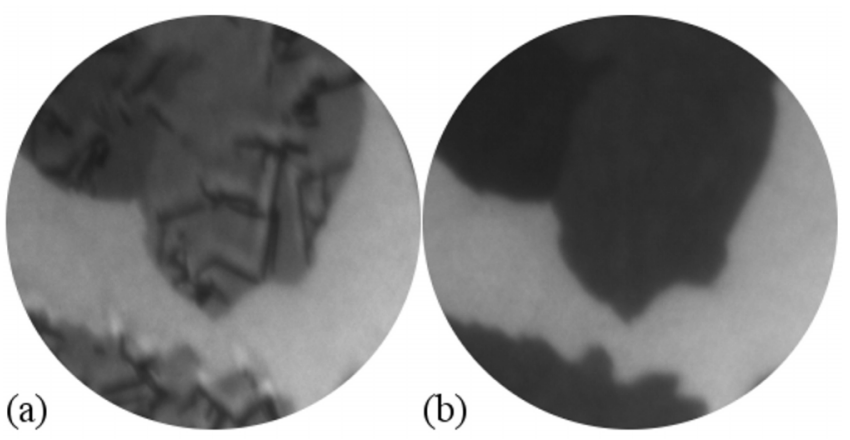

Figure 8.6:

(a, T = 381 K) $26 \mu \mathrm{m}$ FoV PEEM image acquired after stopping the $6 P$ deposition. The $6 P$ needles, on two graphene flakes, can be seen. $(\mathbf{b}, \mathbf{T}=\mathbf{4 0 1} \mathrm{K})$ The same two graphene flakes are cleared from all $6 P$ needles.

immobile (Fig. 8.6(a)). With a further increase of temperature, first the small and later also the bigger needles start to decay until at $400 \mathrm{~K}$ all structures on the flakes have disappeared (Fig. 8.6(b)). The excess molecules can diffuse off the graphene flake into the $2 \mathrm{D}$ gas phase on the supporting $\operatorname{Ir}(111)$ substrate. A further increase of temperature results in a shrinking of the - so far unchanged - irregularly shaped structures on the $\operatorname{Ir}(111)$ surface. Eventually, they all disappear at $416 \mathrm{~K}$. When comparing these results to desorption data obtained on other substrates [27, 19], uncertainties of the temperature measurements in the LEEM sample holder as well as the low heating rate of only $6 \mathrm{~K} / \mathrm{min}$ have to be taken into account. The sequence in which $6 \mathrm{P}$ desorbs from the different substrates is further evidence underlining the weak interaction of $6 \mathrm{P}$ with graphene.

\subsubsection{Deposition of $6 \mathrm{P}$ at $\mathbf{4 0 5} \mathrm{K}$}

Fig. 8.7 is a sequence of images recorded during $6 \mathrm{P}$ deposition at $405 \mathrm{~K}$. Fig. 8.7(a) shows the initial situation. The reflected intensity from the $\operatorname{Ir}(111)$ surface decreases with deposition time indicating the presence of a diluted phase of $6 \mathrm{P}$ on the surface. However, at this elevated temperature neither the formation of a wetting layer, nor the nucleation of any other 6P structure is observed on graphene. We believe, that the already large diffusion length of $6 \mathrm{P}$ at lower temperatures (i.e. as low as $240 \mathrm{~K}$ [13]) will be of the order of the radius of the graphene flakes (roughly $2 \mu \mathrm{m}$ ) at $405 \mathrm{~K}$. As a result, the $6 \mathrm{P}$ molecules diffuse from the flakes onto the $\operatorname{Ir}(111)$ surface where $6 \mathrm{P}$ domains nucleate at the edges of the graphene flake. This process begins after $230 \mathrm{~s}\left(0.146 \mathrm{P} / \mathrm{nm}^{2}\right)$ of deposition (Figs. 8.7(b) and (c)). The contrast is enhanced at centers of fig. 8.7(b,c), which allows to distinguish between the graphene flake (left and brightest), ordered 6P film, and 6P gas phase (upper right). However, the borders between the different areas, in particular in Fig. 8.7(b), are affected by a LEEM image artefact related to abrupt changes in morphology and workfunction [28].

A $\mu$ LEED pattern obtained from the dark band next to graphene flake in fig.8.7(c) on the $6 \mathrm{P}$ covered $\operatorname{Ir}(111)$ surface is shown in Fig. 8.7(d). Only very diffuse spots can be 


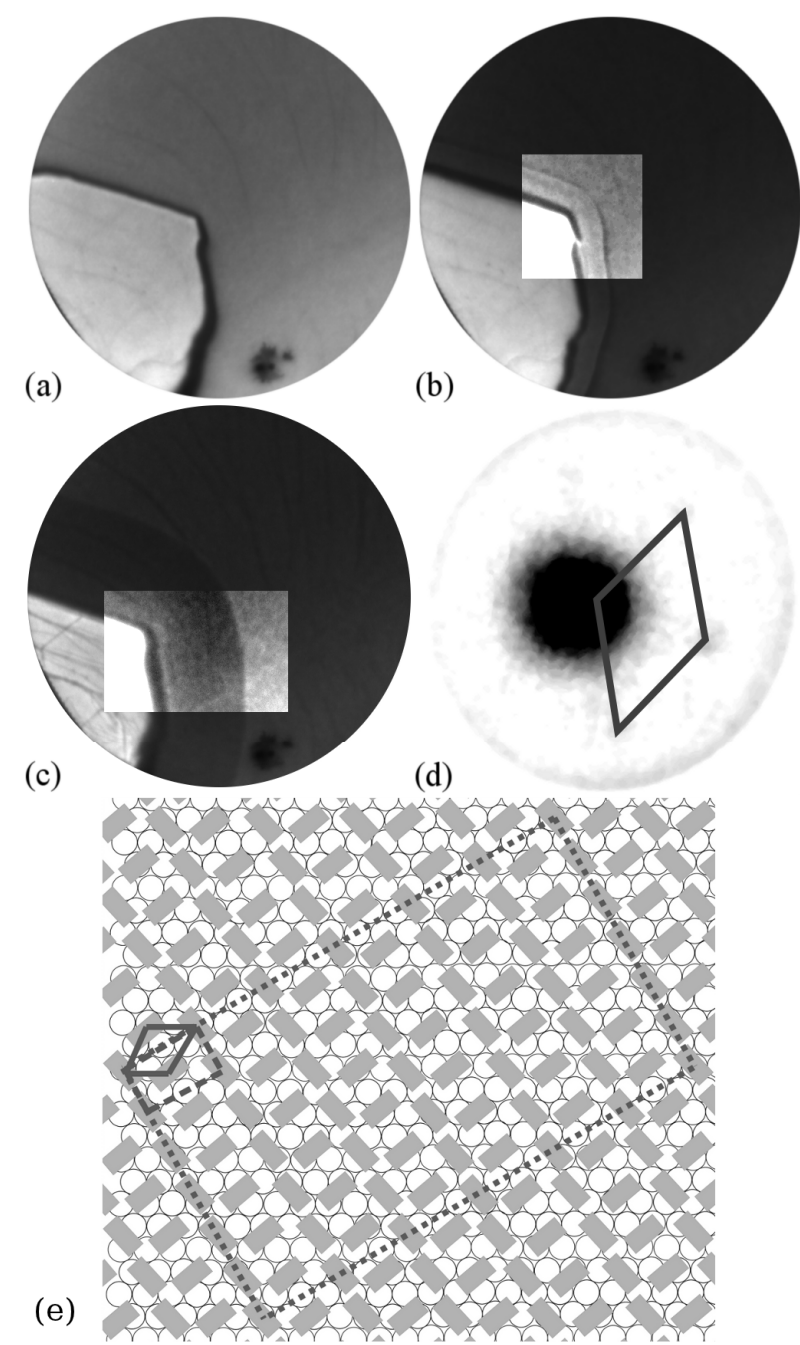

Figure 8.7:

$6 \mu \mathrm{m}$ FoV LEEM images acquired at an electron energy of $2.7 \mathrm{eV}$ and temperature of $405 \mathrm{~K}$. (a, $\mathbf{t}=\mathbf{0} \mathbf{s})$ A graphene flake residing on the Ir(111) surface prior to exposure to $6 P$. Wrinkles and the contours of $\operatorname{Ir}(111)$ surface steps are visible on the single layer graphene flake. $(\mathbf{b}, \mathbf{t}=\mathbf{8 3 1} \mathbf{s}$, $0.536 \mathrm{P} / \mathbf{n m}^{2}$ ) The nucleation of a $6 P$ film takes place on the edges of the graphene flake as is witnessed by the appearance of a band of different intensity at the edge of the graphene (see contrast is enhanced at center). The reflected intensity from the Ir(111) surface decreases. Three different levels of contrast are found. From left to right: graphene, ordered $6 P$ layer, and $6 P$ lattice gas on $\operatorname{Ir}(111)$. ( $\left(\mathbf{c}, \mathbf{t}=\mathbf{1 3 9 1} \mathbf{s}, \mathbf{0 . 8 8} \mathbf{6 P} / \mathbf{n m}^{2}\right)$ The ordered $6 P$ film extending from the graphene flake has grown further (see contrast is enhanced at center). (d) A $\mu L E E D$ pattern is measured at electron energy of $25.6 \mathrm{eV}$ using a $1.4 \mu \mathrm{m}$ field-limiting aperture from the Ir(111) surface area, completely covered with the ordered $6 P$ layer. The nearest neighbor cell is highlighted by red lines. (e) The structural model proposed from the $\mu L E E D$ pattern shown in panel (d). The molecules are arranged in an up-right standing orientation on $\operatorname{Ir}(111)$. The unit cell (dashed lines) and the nearest neighbor cell (solid line) are shown. The $6 \times 6$ superstructure is indicated by a dotted line. Times indicated are measured with respect to the start of $6 P$ deposition. 
found, superimposed on a homogeneous, diffuse background. The crystalline quality of this film is not very high. The nearest neighbor cell highlighted in Fig. 8.7(d) has a size of $5.0 \AA$ by $5.0 \AA$ with an angle $\beta$ of $120^{\circ}$. The obvious way to accommodate the $6 \mathrm{P}$ molecules into such a small space is in an upright standing way where the long molecular axis is roughly perpendicular to the substrate. Using the unit cell of the bulk (100) plane $\left(8.091 \AA\right.$ by $5.568 \AA$ and $\beta=90^{\circ}$ [17]) as a starting point, we can deduct the unit cell of $6 \mathrm{P}$ on $\operatorname{Ir}(111)$ to be $8.7 \AA$ by $5 \AA$ and $\beta$ and $\Theta=90^{\circ}$ (dashed line in Fig. 8.7(e)). Compared to the bulk structure, this unit cell is compressed along the short axis. The resulting matrix notation of the overlayer with respect to the underlying $\operatorname{Ir}(111)$ is given by the following quasiepitaxial coincidence type II relationship [16] ( $\left.\begin{array}{cc}1.9 & 3.7 \\ 1.9 & 0\end{array}\right)$. Using the same arguments as for the previous structures, a $6 \times 6$ superstructure describes the situation more precisely and results in the following matrix notation $\left(\begin{array}{ll}11 & 22 \\ 11 & 0\end{array}\right)$ which is depicted in Fig. 8.7(e). The distortion of the 6P unit cell is geometrically justified as the molecular rows will have the substrate dictated $120^{\circ}$ angle. $\mu$ LEED patterns obtained far away from the flakes show only the disordered two-dimensional gas phase of 6P. Different to the well investigated [29-31], but non-metallic system - 6P on $\mathrm{TiO}_{2}-$ we see no evidence for an additional ordered layer of flat lying molecules [32].

In general, increased substrate temperatures have been identified as one of the reasons for the growth of up-right standing $6 \mathrm{P}$ molecules $[7,33,34]$. In the same way, the elevated surface temperature of $\operatorname{Ir}(111)$ favors the growth of up-right standing $6 \mathrm{P}$ thin films. No other structures - neither on $\operatorname{Ir}(111)$ nor on graphene - were found for this deposition temperature.

\subsection{Conclusions}

The deposition of 6P molecules and growth of 6P structures on graphene has been studied at different temperatures. For sample temperatures during deposition up to $352 \mathrm{~K}$, wrinkles in the graphene act as preferential nucleation sites for both, a (1 $\overline{11})$ wetting layer and 6P needles with the same crystallographic orientation. The 6P needles form after the completion of the wetting layer. This is usually identified as the Stranski-Krastanov growth mode, often observed for 6P films formed from flat lying molecules [3].

Defects of the $\operatorname{Ir}(111)$ substrate - a result of carbon residues after the formation of the graphene flakes - are nucleation sites for the growth of ramified structures consisting of upright standing 6P molecules. However, with increasing sample temperature (compare fig. 8.1(d) at $320 \mathrm{~K}$ to fig. 8.5(a) at $352 \mathrm{~K}$ ), less but longer 6P needles are formed on graphene. In addition, $6 \mathrm{P}$ nucleation on the $\operatorname{Ir}(111)$ surface gets increasingly difficult and ramified islands of upright $6 \mathrm{P}$ are exclusively nucleated at the rim of graphene flakes. Further increase of the deposition temperature to $405 \mathrm{~K}$ results in a considerable change of growth behavior. Neither a wetting layer, nor any three dimensional needles are observed on graphene. A 6P(100) layer does, however, nucleate at the edges of the graphene flakes. It grows on the $\operatorname{Ir}(111)$ surface in a step flow-like fashion. This layer built from upright standing molecules shows poor crystallinity.

Our study illustrates that at all temperatures investigated, the growth behavior of $6 \mathrm{P}$ on graphene and $\operatorname{Ir}(111)$ is governed by defects. Up to $352 \mathrm{~K}$, graphene wrinkles dictate 
the nucleation and growth behavior of the $6 \mathrm{P}$ wetting layer and needles. At $405 \mathrm{~K}$, the edges of the graphene flakes are the sites where 6P domains develop on $\operatorname{Ir}(111)$. 


\section{Bibliography}

[1] A. Niko, F. Meghdadi, C. Ambrosch-Draxl, P. Vogl, and G. Leising, Synth. Met. 76 (1996), 177.

[2] T. Mikami and H. Yanagi, Appl. Phys. Lett. 73 (1998), 563.

[3] C. Teichert, G. Hlawacek, Andreev, H. Sitter, P. Frank, A. Winkler, and N. S. Sariciftci, Appl. Phys. A-Mater 82 (2006), 665.

[4] G. Hlawacek, P. Puschnig, P. Frank, A. Winkler, C. Ambrosch-Draxl, and C. Teichert, Science 321 (2008), 108.

[5] F. Yang, M. Shtein, and S.R. Forrest, Nat. Mater. 4 (2004), 37.

[6] E.J. Kintzel, D.M. Smilgies, J.G. Skofronick, S.A. Safron, and D.H. van Winkle, J. Vac. Sci. Technol. A 22 (2004), 107.

[7] S. Müllegger, G. Hlawacek, T. Haber, P. Frank, C. Teichert, R. Resel, and A. Winkler, Appl. Phys. A-Mater 87 (2007), 103.

[8] O. Lengyel, A. Šatka, T. Haber, Kovać, H. Sitter, and R. Resel, Cryst. Res. Technol. 43 (2008), 44.

[9] E. Bauer, Surf. Rev. Lett. 5 (1998), 1275.

[10] E. Bauer, Rep. Prog. Phys. 57 (1994), 895.

[11] J. Coraux, A.T. N'Diaye, M. Engler, C. Busse, D. Wall, N. Buckanie, F.J. Meyer zu Heringdorf, R. van Gastel, B. Poelsema, and T. Michely, New J. Phys. 11 (2009), 023006.

[12] A.T. N'Diaye, R. van Gastel, A.J. Martínez-Galera, J. Coraux, H. Hattab, D. Wall, F.-J. Meyer zu Heringdorf, M. Horn von Hoegen, J.M. Gómez-Rodríguez, B. Poelsema, C. Busse, and T. Michely, New J. of Phys. 11 (2009), 113056.

[13] G. Hlawacek, F.S. Khokhar, R. van Gastel, B. Poelsema, and C. Teichert, Nano Lett. 11 (2011), 333.

[14] R. van Gastel, A. T. N’Diaye, D. Wall, J. Coraux, C. Busse, N.M. Buckanie, F.J. Meyer zu Heringdorf, M. Horn von Hoegen, T. Michely, and B. Poelsema, Appl. Phys. Lett. 95 (2009), 121901.

[15] G. Hlawacek, F.S. Khokhar, R. van Gastel, B. Poelsema, and C. Teichert, IBM J. Res. Dev. 55 (2011), 15.

[16] D.E. Hooks, T. Fritz, and M.D. Ward, Adv. Mater. 13 (2001), 227.

[17] K. Baker, A. Fratini, T. Resch, H. Knachel, W. Adams, E. Socci, and B. Farmer, Polymer 34 (1993), 1571.

[18] B. Winter, J. Ivanco, F.P. Netzer, and M.G. Ramsey, Thin Solid Films 433 (2003), 269. 
[19] S. Müllegger and A. Winkler, Surf. Sci. 600 (2006), 1290.

[20] G. Hlawacek, C. Teichert, S. Müllegger, R. Resel, and A. Winkler, Synth. Met. 146 (2004), 383.

[21] R. Resel, M. Oehzelt, T. Haber, G. Hlawacek, C. Teichert, S. Müllegger, and A. Winkler, J. Cryst. Growth 283 (2005), 397.

[22] B. Winter, S. Berkebile, J. Ivanco, G. Koller, F.P. Netzer, and M.G. Ramsey. Appl. Phys. Lett. 88 (2006), 253111.

[23] R. Strayer, W. MacKie, and L. Swanson, Surf. Sci. 34 (1973), 225.

[24] E. Starodub, A. Bostwick, L. Moreschini, S. Nie, F.E. Gabaly, K.F. McCarty, and E. Rotenberg, Phys. Rev. B 83 (2011), 125428.

[25] A.J. Fleming, F.P. Netzer, and M.G. Ramsey, J. Phys.: Condens. Matter 21 (2009), 445003.

[26] F. Balzer and H.G. Rubahn, Surf. Sci. 548 (2004), 170.

[27] B. Winter, J. Ivanco, F.P. Netzer, M.G. Ramsey, I. Salzmann, and R. Resel, Langmuir 20 (2004), 7512.

[28] F. Schertz, D. Kutnyakhov, S. Schuppler, P. Nagel, S. Nepijko, and G. Schönhense, Appl. Phys. A 102 (2011), 253.

[29] R. Resel, M. Oehzelt, O. Lengyel, T. Haber, T. Schulli, A. Thierry, G. Hlawacek, C. Teichert, S. Berkebile, and G. Koller, Surf. Sci. 600 (2006), 4645.

[30] G. Hlawacek, C. Teichert, A.Y. Andreev, H. Sitter, S. Berkebile, G. Koller, M. Ramsey, and R. Resel, Phys. Stat. Sol. (A) 202 (2005), 2376.

[31] S. Berkebile, G. Koller, G. Hlawacek, C. Teichert, F. P. Netzer, and M. G. Ramsey, Surf. Sci. 600 (2006), L313.

[32] L. Sun, S. Berkebile, G. Weidlinger, G. Koller, M. Ho- hage, F.P. Netzer, M. G. Ramsey, and P. Zeppenfeld, Phys. Chem. 12 (2010), 3141.

[33] L. Athouel, G. Froyer, R. Resel, N. Koch, F. Megh- dadi, and G. Leising, Synth. Met. 101 (1999), 627.

[34] T. Haber, S. Müllegger, A. Winkler, and R. Resel, Phys. Rev. B 74 (2006), 045419 . 


\section{Summary}

The topic of this thesis is the molecular functionalization of substrates. A novel approach in which organic molecules are used to grow two dimensional structures was employed to achieve this goal. Low Energy Electron Microscopy (LEEM) and Micro Low Energy Electron diffraction ( $\mu$ LEED) are the two techniques that were used to investigate the functionalized substrates. Three organic molecules, trimesic acid (TMA), 4,4'-biphenyldicarboxylic acid (BDA), and para-sexiphenyl (6P), were used, however, the majority of the investigations was done with BDA and 6P. Metal $(\mathrm{Cu}(001), \operatorname{Ir}(111))$ and graphene substrates have been used to conduct the growth investigations.

In Chapter 3, some initial results are outlined. After observing the evolution of the area of TMA domains at low temperature and at room temperature, it was evident that a better choice of the molecule for the experiments was required to form domains of a size that is observable with LEEM. Larger domains also provide more opportunities to analyze the growth in the framework of competing interactions, e.g. by investigating domain boundary fluctuations. Moreover, the formation of molecular domains on the $\mathrm{Cu}(001)$ surface leads to a significant change of the work function. The resulting fields that exist at the boundaries of the molecular domains will make any kind of quantitative analysis impossible if the size of the domains is small with respect to the distorting effects. The role of the imaging electrons in causing possible radiation damage to the organic thin films is also discussed. To limit the radiation damage, specifically at high energies, LEED measurements must always be performed in a rapid fashion and equal care has to be taken in order to minimize the exposure of organic films to the beam during imaging at low energies. We also concluded from the TMA/Cu(001) experiments that LEEM is indeed the most appropriate instrument for the kind of investigations that we have conducted.

To circumvent the issues with TMA growth and tailor the balance of the forces that are relevant to self-assembly, a bigger molecule was used in subsequent experiments and the results are shown in Chapter 4. This chapter describes the growth of BDA on 
$\mathrm{Cu}(001)$ which has been studied with LEEM and $\mu$ LEED. The emergence of large islands and hydrogen bonding of molecules to other, perpendicularly oriented, adjacent molecules was confirmed. In the molecular islands, the adjacent molecules are oriented in a perpendicular fashion and their interaction is primarily conveyed through hydrogen stabilized bonds. $\mu$ LEED revealed that the benzene rings are twisted along the intramolecular axis. Growth at room temperature showed unconventional nucleation behavior: Nucleation occurs late and the subsequent growth of BDA-domains was interrupted when the islands reach a linear size of about 90 base units. This feature was attributed to the accumulation of (tensile) stress which was no longer present at $448 \mathrm{~K}$. Classic Ostwald ripening was observed in island ensembles at $373 \mathrm{~K}$. The decay of a large island at $448 \mathrm{~K}$ in a well-defined geometry showed scaling behavior with a time exponent $\alpha=0.61 \pm 0.05$. This is indicative of diffusion limited decay, in agreement with the also observed Ostwald ripening features.

In Chapter 5, the role of substrate in growth of BDA molecules structures was explored by replacing $\mathrm{Cu}(001)$ with the technologically relevant graphene surface. The graphene was first grown on an $\operatorname{Ir}(111)$ substrate and, later on, BDA chains were grown and studied. Bright-field LEEM images provided direct insight in the growth dynamics and showed that defects, wrinkles, in the graphene play a crucial role, both during the nucleation phase and in the final morphology of the molecular film that forms. The origin of the inhomogeneous nucleation of BDA domains was speculated to be the strain relaxation that occurs at the wrinkles. $\mu$ LEED measurements revealed that the BDA domains that formed were ordered, hydrogen bond stabilized, chain structure. Dark field LEEM measurements revealed that a continuous film of BDA consists of numerous different rotational domains that transgress wrinkles and bumps originating from substrate steps. Domain boundaries were observed in those locations where growing domains had coalesced. The films that were grown were stable over extended periods of time at room temperature and slightly above. Above $350 \mathrm{~K}$, decay of the BDA domains was observed. The edges of a graphene flake were shown to act as an additional source of molecules for extended times even when BDA was no longer deposited from the vapor phase. BDA domains that were present at the top side of a graphene flake edge were shown to assist in the upward diffusion of BDA onto the graphene flake. It is important to state that the use of graphene as a substrate for self-assembly of molecular networks opens up a new applications area by combining the principles of two-dimensional organic self-assembly with the remarkable electronic properties of graphene. It offers enormous possibilities when using custom-made molecules that alter the molecule-molecule or molecule-graphene interaction.

Having investigated the growth and structures of BDA molecules on $\mathrm{Cu}(001)$ and graphene surfaces, we replaced the BDA molecule with another organic semiconductor molecule, 6P. We studied the layer-by-layer growth of the organic semiconductor molecule $6 \mathrm{P}$ on the graphene. $6 \mathrm{P}$ has a longer backbone than BDA and is anticipated to form more complex structures when grown on flat, defect-free substrates. The growth of atomically smooth layers of 6P at low temperature on a graphene substrate was demonstrated and described in Chapter 6. Initially, small islands formed. An open structure consisting of only flat-lying molecules was found as an initial structure for the first layer with $\mu$ LEED. This layer then transformed into a complete monolayer 
through the addition of interdigiting, edge-on molecules that result in a bulk-like arrangement of the molecules. Subsequent layers were formed by a repetition of this cycle as we found an adlayer with an open structure similar to what was found for the initial layer, covering the surface of thicker films. Up to at least 4.35 ML, the growth continues in this layer-by-layer fashion. This growth mode can be used to form films with a high charge carrier mobility and good overall device performance. It is an enabler for future organic, flexible, and low cost devices.

The growth of high-quality films of conjugated molecules with smooth interfaces is important to assist the advent of organic electronics. To further the understanding of the growth of 6P films, the diffusion process of 6P molecules on graphene flakes and on iridium were studied and described in Chapter 7. The initial island formation on graphene was characterized by a high mobility of 6P molecules. This allowed for strain driven shape and position changes of the initial submonolayer islands. A delicate interplay between intrinsic strain in the graphene flakes and strain that builds up with increasing island size was considered as one of the possible driving forces. However, electronic effects based on charge transfer between 6P and graphene, and, graphene and iridium are another possible explanation. The growth of $6 \mathrm{P}$ on the $\operatorname{Ir}(111)$ surface yielded ramified islands formed by upright standing molecules. Using capture zone scaling, we obtained a critical nucleus size of $i=0$. This result was interpreted as an indication for heterogeneous nucleation triggered by a high density of defects. The defects are most likely carbon clusters left over from the graphene formation or possibly small graphene flakes. As long as the trap binding energy of the defects for $6 \mathrm{P}$ is sufficiently high, a homogeneous nucleation with cluster size between 2 and 3 is suppressed. In addition, we presented possible configurations for the critical nucleus for $i=0$ to $i=4$. Only the last one has a comparatively small number of fully exposed $\pi$ systems. We interpret this in terms of a reduced stability for the intermediate nuclei. In a scenario where nucleation is mostly defect driven, these cluster sizes are most likely suppressed due to dominant trapping at defect sites.

To complete our study of the behavior of organic 6P films on $\operatorname{Ir}(111)$ supported graphene flakes, we studied the growth for various substrate temperatures in real-time with LEEM in Chapter 8. $\mu$ LEED was used to determine the structure of the different $6 \mathrm{P}$ features formed on the surface. The deposition of $6 \mathrm{P}$ molecules and growth of $6 \mathrm{P}$ structures on graphene was studied at different temperatures. For sample temperatures during deposition up to $352 \mathrm{~K}$, wrinkles in the graphene acted as preferential nucleation sites for both, a $6 \mathrm{P}(111)$ wetting layer and $6 \mathrm{P}$ needles with the same crystallographic orientation. The $6 \mathrm{P}$ needles formed after the completion of the wetting layer. This is usually identified as the Stranski-Krastanov growth mode, often observed for 6P films formed from flat lying molecules. Defects at the $\operatorname{Ir}(111)$ substrate - a result of carbon residues after the formation of the graphene flakes - are nucleation sites for the growth of ramified structures consisting of upright standing 6P molecules. However, with increasing sample temperature, less but longer 6P needles formed on graphene. In addition, 6P nucleation on the $\operatorname{Ir}(111)$ surface became increasingly difficult and ramified islands of upright $6 \mathrm{P}$ exclusively nucleated at the rim of graphene flakes. A further increase of the deposition temperature to $405 \mathrm{~K}$ resulted in a considerable change of growth behavior. Neither a wetting layer nor three dimensional needles were observed 
on graphene. A $6 \mathrm{P}(100)$ layer did, however, nucleate at the edges of the graphene flakes. It grew on the $\operatorname{Ir}(111)$ surface in a step flow-like fashion. This layer built from upright standing molecules exhibited poor crystallinity. Our study illustrated that at all temperatures investigated, the growth behavior of $6 \mathrm{P}$ on graphene and $\operatorname{Ir}(111)$ is governed by defects. Up to $352 \mathrm{~K}$, graphene wrinkles dictated the nucleation and growth behavior of the $6 \mathrm{P}$ wetting layer, and needles. At $405 \mathrm{~K}$, the edges of the graphene flakes were the sites where 6P domains developed on $\operatorname{Ir}(111)$.

In summary, in this thesis we have successfully investigated the role of moleculesubstrate, molecule-molecule interaction, and substrate temperature on growth and final structures formed by simple conjugated molecules on metal and graphene substrates. We also demonstrated that the growth process and structures of organic molecules can be characterized in-depth with real-time LEEM and $\mu$ LEED, respectively. 


\section{Samenvatting}

Dit proefschrift beschrijft onderzoek aan de moleculaire functionalisering van oppervlakken. Daartoe werden organische moleculen ingezet om twee-dimensionale structuren te groeien. De gefunctionaliseerde oppervlakken zijn met behulp van LEEM (Low Energy Electron Microscopy) en $\mu$ LEED (Micro Low Energy Electron Diffraction) geanalyseerd. Drie verschillende organische moleculen, te weten: (trimesinezuur zuur) TMA, 4,4 '-biphenyldicarboxylic zuur (BDA) en para-sexiphenyl (6P), zijn onderzocht. Het merendeel van het onderzoek is gedaan aan BDA en 6P. $\mathrm{Cu}(001)$ en $\operatorname{Ir}(111)$ en grafeen op $\operatorname{Ir}(111)$ zijn gebruikt als substraat voor de groei van de moleculaire films.

In hoofdstuk 3 zijn enkele eerste resultaten beschreven. De ontwikkeling van TMAdomeinen bij lage temperatuur en helaas ook bij kamertemperatuur maakte het duidelijk, dat de experimenten een ander molecuul vereisen om domeinen te vormen, die voldoende groot zijn om waar te nemen met LEEM. Grotere domeinen zijn noodzakelijk om de groei te analyseren in het kader van concurrerende interacties, bijvoorbeeld door het onderzoeken van de fluctuaties van domeinwanden. Bovendien leidt de vorming van moleculaire domeinen op een $\mathrm{Cu}(001)$ oppervlak tot een significante verandering van de werkfunctie. De resulterende velden aan de grenzen van de moleculaire domeinen maakt een kwantitatieve analyse niet mogelijk als de grootte van de domeinen klein is ten opzichte van deze storende effecten. De rol van de afbeeldende elektronen bij het eventueel veroorzaken van eventuele stralingsschade aan de organische dunne films wordt ook besproken. Ter beperking van de schade door straling, in het bijzonder bij hoge energien, moeten LEED metingen altijd in een zo kort mogelijke tijd worden uitgevoerd. Een vergelijkbare zorgvuldigheid moet worden betracht om de blootstelling van organische films aan de elektronenbundel tijdens de beeldvorming bij lage energien te minimaliseren. We trekken uit de TMA / $\mathrm{Cu}(001)$ experimenten de conclusie dat LEEM inderdaad het meest geschikte instrument is voor het soort onderzoek dat we hebben uitgevoerd.

Voor het omzeilen van de voornoemde problemen met TMA groei en ten behoeve van het aanpassen van de balans van de krachten die relevant zijn voor zelf-assemblage, is een groter molecuul gebruikt in de volgende experimenten. De resultaten daarvan zijn weergegeven in hoofdstuk 4, dat de groei van BDA op $\mathrm{Cu}(001)$ beschrijft. De in de literatuur gerapporteerde groei van deze moleculen in grote eilanden werd bevestigd. In de moleculaire eilanden, zijn aangrenzende moleculen loodrecht georinteerd en hun interactie wordt voornamelijk verzorgd via waterstofbruggen. Uit $\mu$ LEED metingen is gebleken dat de benzeenringen gedraaid zijn langs de intramoleculaire as. De groei bij kamertemperatuur liet onconventioneel kiemgedrag zien: Nucleatie gebeurt in een laat stadium en de daaropvolgende groei van de BDA-domeinen hield op wanneer de eilanden een lineaire afmeting van ongeveer 90 basiseenheden bereikten. Dit gedrag werd toegeschreven aan de accumulatie van (trek)spanning, die niet meer aanwezig is bij $448 \mathrm{~K}$. Klassieke Ostwald rijping werd waargenomen voor eiland-ensembles bij 373 $\mathrm{K}$. Het verval van een groot, gesoleerd eiland bij $448 \mathrm{~K}$ vertoonde schalingssgedrag met een karakteristieke exponent $\alpha=0.61 \pm 0.05$. Deze exponent is een indicatie voor z.g. diffusie-gelimiteerd verval, in overeenstemming met eveneens waargenomen Ostwald rijpingsverschijnselen. 
In hoofdstuk 5, is de rol van het substraat in de groei van de moleculaire BDAstructuren onderzocht door het $\mathrm{Cu}(001)$ oppervlak te vervangen door het technologisch relevante grafeen oppervlak. Het grafeen werd eerst gegroeid op een Ir (111) substraat en later werden BDA ketens hierop aangebracht en bestudeerd. "Bright-field" LEEM beelden, die direct inzicht geven in de groeidynamiek, toonden aan dat defecten (kreukels) in het grafeen een cruciale rol spelen, zowel tijdens de nucleatie fase als bij de uiteindelijke morfologie van de zich vormende moleculaire film. De oorsprong van de inhomogene nucleatie van BDA domeinen werd gevonden in de spanningsrelaxatie die zich voordoet op de kreukels. Uit $\mu$ LEED metingen blijkt, dat de zich vormende BDA domeinen uit een waterstofbrug gestabiliseerde ketenstructuur bestaan. Uit "dark-field" LEEM metingen bleek dat een continue film van BDA bestaat uit een groot aantal verschillende rotatie-domeinen, die kreukels en oneffenheden samenhangend met de aanwezige substraatstappen, overschrijden. Domeingrenzen werden waargenomen op die plaatsen waar de domeinen samenkomen. De films waren gedurende langere tijd stabiel bij kamertemperatuur en iets daarboven. Boven $350 \mathrm{~K}$ is het verval van de BDA domeinen waargenomen. De randen van een grafeenlaag bleken gedurende lange tijd te fungeren als een extra bron van moleculen, zelfs als BDA niet langer vanuit de dampfase werd aangeboden. BDA domeinen die aanwezig waren aan de bovenkant van een rand van zo'n grafeenvlok versnellen de opwaartse stroom van BDA naar het grafeen. Het is belangrijk te vermelden dat het gebruik van grafeen als een substraat voor zelf-assemblage van moleculaire netwerken een nieuwe reeks toepassingen en toepassingsgebieden opent door het combineren van de principes van twee-dimensionale organische zelf-assemblage met de opmerkelijke elektronische eigenschappen van grafeen. Het biedt uitgebreide mogelijkheden bij het gebruik van geselecteerde moleculen met speciale molecuul-molecuul of molecuul-grafeen interacties.

$\mathrm{Na}$ het onderzoeken van de groei en de structuren van BDA moleculen op $\mathrm{Cu}$ (001) en op grafeenoppervlakken, werd het BDA molecuul door een ander organisch halfgeleider molecuul, 6P, vervangen. Opmerkelijk genoeg vonden we laag-voor-laag groei van de organische halfgeleider 6P op grafeen. 6P heeft een langere "backbone" dan BDA en het vormt naar verwachting meer complexe structuren op vlakke, defectloze substraten. De groei van atomair gladde lagen van 6P bij lage temperatuur op een grafeen substraat wordt beschreven in hoofdstuk 6 . In eerste instantie werden kleine eilanden gevormd. Met behulp van $\mu$ LEED konden we aantonen dat het oppervlak eerst geheel bedekt wordt door plat gelegen moleculen. In een tweede stap wordt de eerste laag gecompleteerd door om en om rijen van om hun lengte as gekantelde moleculen toe te voegen. Deze structuur komt globaal overeen met de bekende bulk structuur van een uit 6P opgebouwd kristal. De groei van volgende lagen volgt door herhaling van deze cyclus met eerst een verdunde laag met plat liggende moleculen gevolgd door een verdichtingsfase, waarin de ketens met liggende moleculen om en om worden afgewisseld met gekantelde rijen moleculen. Dit spel herhaalde zich tot tenminste 4.35 ML, d.w.z. 6P groeit initieel op grafeen in een laag-voor-laag modus. Deze niet eerder gerapporteerde groei modus kan worden gebruikt om films met een hoge ladingsdragermobiliteit en goede algemene prestaties te vormen. Het is mogelijk een goedkope en flexibele "enabler" voor toekomstig gebruik van zulke moleculaire 
dunne films voor toepassingen in b.v. sensoren en lichtbronnen.

De groei van goed gedefinieerde films van geconjugeerde moleculen met gladde interfaces is belangrijk om de komst van organische elektronica te versnellen. Om ons begrip van de groei van de 6P films te vergroten, werd het diffusieproces van $6 \mathrm{P}$ moleculen op grafeen lagen en op iridium bestudeerd. De resultaten daarvan zijn beschreven in hoofdstuk 7. De initile eilandvorming op grafeen wordt gekenmerkt door een hoge mobiliteit van de 6P moleculen. Dit leidde tot spanningsgestabiliseerde vormen en positieveranderingen van de eerste submonolaag eilanden. Een delicaat samenspel tussen intrinsieke spanning in het grafeen en de spanning die opbouwt bij toenemende eilandgrootte werd beschouwd als een van de mogelijke drijvende krachten. Echter, elektronische effecten op basis van ladingsoverdracht tussen 6P en grafeen en grafeen en iridium bieden een andere mogelijke verklaring. De groei van $6 \mathrm{P}$ op $\operatorname{Ir}(111)$ leverde vertakte eilanden op, gevormd door rechtopstaande moleculen. Met behulp van een z.g. "capture zone" analyse verkregen we een kritische kern- grootte van $i=0$. Dit resultaat werd genterpreteerd als een indicatie voor heterogene nucleatie, veroorzaakt door een hoge dichtheid aan defecten. De defecten bestaan hoogstwaarschijnlijk uit koolstof(clusters), gevormd door kleine grafeenvlekken of door onvolledig gesplitste koolwaterstoffen. Zolang de bindingsenergie van de defecten voor 6P hoog genoeg is, wordt een homogene nucleatie met een clustergrootte tussen 2 en 3 moleculen onderdrukt. Daarnaast hebben we mogelijke configuraties gesuggereerd voor de kritische kernen met grootte $i=0$ tot $i=4$. Alleen de laatste heeft een relatief klein aantal volledig blootgestelde ?-systemen. We interpreteren dit in termen van een verminderde stabiliteit voor de tussenliggende kernen. In een scenario waarin nucleatie meestal defect gedreven is, worden deze clusters waarschijnlijk onderdrukt als gevolg van dominerende verbijfstijden op roosterplaatsen met een niet ideale omgeving (defecten).

Verder bestudeerden we met behulp van LEEM het gedrag van organische 6P films op grafeen tijdens hun groei. Zoals beschreven in hoofdstuk 8, werd $\mu$ LEED gebruikt om de structuur van de verschillende op het oppervlak gevormde 6P-formaties te bepalen. Hun nucleatie en groei op grafeen werd bestudeerd bij verschillende temperaturen van het $\operatorname{Ir}(111)$-substraat. Voor temperaturen tijdens depositie van maximaal 352 $\mathrm{K}$, fungeerden kreukels in het grafeen als preferente groeikernen voor zowel de z.g. 6P(111) "wetting"-laag als de 6P naalden met dezelfde kristallografische orintatie. De $6 \mathrm{P}$ naalden werden pas gevormd na voltooiing van de wettinglaag. Deze groei modus wordt meestal aangeduid met Stranski-Krastanov groei en wordt vaak waargenomen voor 6P films gevormd uit plat liggende moleculen. Defecten op het $\operatorname{Ir}(111)$ substraat - een gevolg van koolstof resten na de vorming van het grafeen - vormen kiemplaatsen voor de groei van sterk vertakte structuren, die bestaan uit rechtop staande $6 \mathrm{P}$ moleculen. Met toenemende substraat temperatuur vormen zich minder, maar langere 6P naalden op grafeen. Daarnaast werd de 6P nucleatie op $\operatorname{Ir}(111)$ steeds moeilijker en werden wijdvertakte eilanden van rechtopstaand $6 \mathrm{P}$ uitsluitend aan de rand van grafeen domeinen gevormd. Een verdere toename van de depositie temperatuur tot $405 \mathrm{~K}$ leidde tot een aanzienlijke verandering van het groeigedrag. Noch een wetting laag, noch driedimensionele naalden werden waargenomen op grafeen. Een 6P(100) laag, ontsproot aan de randen van de grafeen domeinen. Het groeide op $\operatorname{Ir}(111)$ via de propagatie 
van stappen. Deze laag, opgebouwd uit rechtopstaande moleculen laat een slechte kristalliniteit zien. Onze studie toonde aan dat bij alle onderzochte temperaturen, het groeigedrag van 6P op grafeen en $\operatorname{Ir}(111)$ wordt benvloed door defecten. Tot $352 \mathrm{~K}$ dicteren rimpels in het grafeen het kiem- en groeigedrag van zowel de 6P wettinglaag als de naalden. Bij $405 \mathrm{~K}$ zijn de randen van het grafeen de plaatsen waar 6P-domeinen zich ontwikkelen op $\operatorname{Ir}(111)$.

Samenvattend hebben we in dit proefschrift met succes onderzoek gedaan naar de rol van de molecuul-substraat, molecuul-molecuul interacties en de invloed van de temperatuur van het substraat op de groei en de resulterende structuren die gevormd worden door eenvoudige geconjugeerde moleculen op metaal- en grafeensubstraten. We hebben aangetoond dat het groeiproces en de structuren van organische moleculen kunnen worden gekarakteriseerd met, respectievelijk, real-time LEEM en $\mu$ LEED. 


\section{Acknowledgements}

I would like to take this opportunity to say thanks to several people, whom help and cooperation has made this thesis possible.

First of all, I would like to say thanks to my supervisor, Prof. dr. ir. Bene Poelsema. It is difficult to overstate my gratitude for him. His continuous guidance and support has made this thesis possible.

I am indebted to say thanks to my daily supervisor, Dr. Raoul van Gastel for useful discussions, academic, and practical support, and his confidence in me. Thanks Raoul for always being there for me.

I would like to thank Dr. Gregor Hlawacek for his support and useful discussions. I enjoyed working with him in final stages of this project.

I would like to thank my colleagues, Robert and Tjeerd, with whom I shared LEEM Lab. We shared technical experiences and that helped me to learn new things.

My special thanks for prof.dr. A. van Silfhout for useful discussions and always managing free time for me.

I would like to thank my group members, Prof. dr. ir. H.J.W. Zandvliet, Dr. ir. Herbert Wormeester, Dr. ir. Stefan Kooij, and PhD students, Muhammad Akram Raza, Daniel Schwarz, Arzu Colak, Tijs Mocking, Avijit Kumar, Maciej Jankowski, Vasilisa Veligura, Robin Berkelaar, Patrick Jansen, Chris Hellenthal, Ren Heimbuch, Hande Cingil, and Hairong $\mathrm{Wu}$.

I am grateful to the Rianne Nales, Hans Bevers, and Herman Oerbekke for sorting out the bureaucratic and technical challenges smoothly.

I say thanks to my family for providing a supporting environment.

My brothers, Zeeshan, Arslan, my loving sister Arzoo, and my wife Hina. Last but not the least, I want to say thanks to my parents. They raised me, supported me, and loved me unconditionally.

I dedicate this thesis to my Loving Parents. 


\section{Curriculum Vitae}

Fawad Salman Khokhar was born on 28 January, 1979 in Gujranwala, Pakistan. He finished his secondary school in 1994. He decided to study Physics, Chemistry, and Mathematics in higher secondary school. He obtained his Bachelor degree in 2000 from University of Punjab, Lahore, Pakistan, with major in Physics and Mathematics. He did Master of Science in Physics, in 2003, from Quaid-e-Azam University, Islamabad, Pakistan. His interest in experimental material science moved him to Linkoping University, Sweden where he obtained his Master of Science degree in Material Science and Nanotechnology in 2005.

In 2005, he started PhD studies in Solid State Physics group (now known as Physics of Interfaces and Nanomaterials) of Prof. dr. ir. B. Poelsema at University of Twente, Enschede, The Netherlands. His $\mathrm{PhD}$ research is focused on the growth of organic molecular films on metal and graphene surfaces and study with LEEM. The results accomplished during this project are presented in this $\mathrm{PhD}$ thesis. 


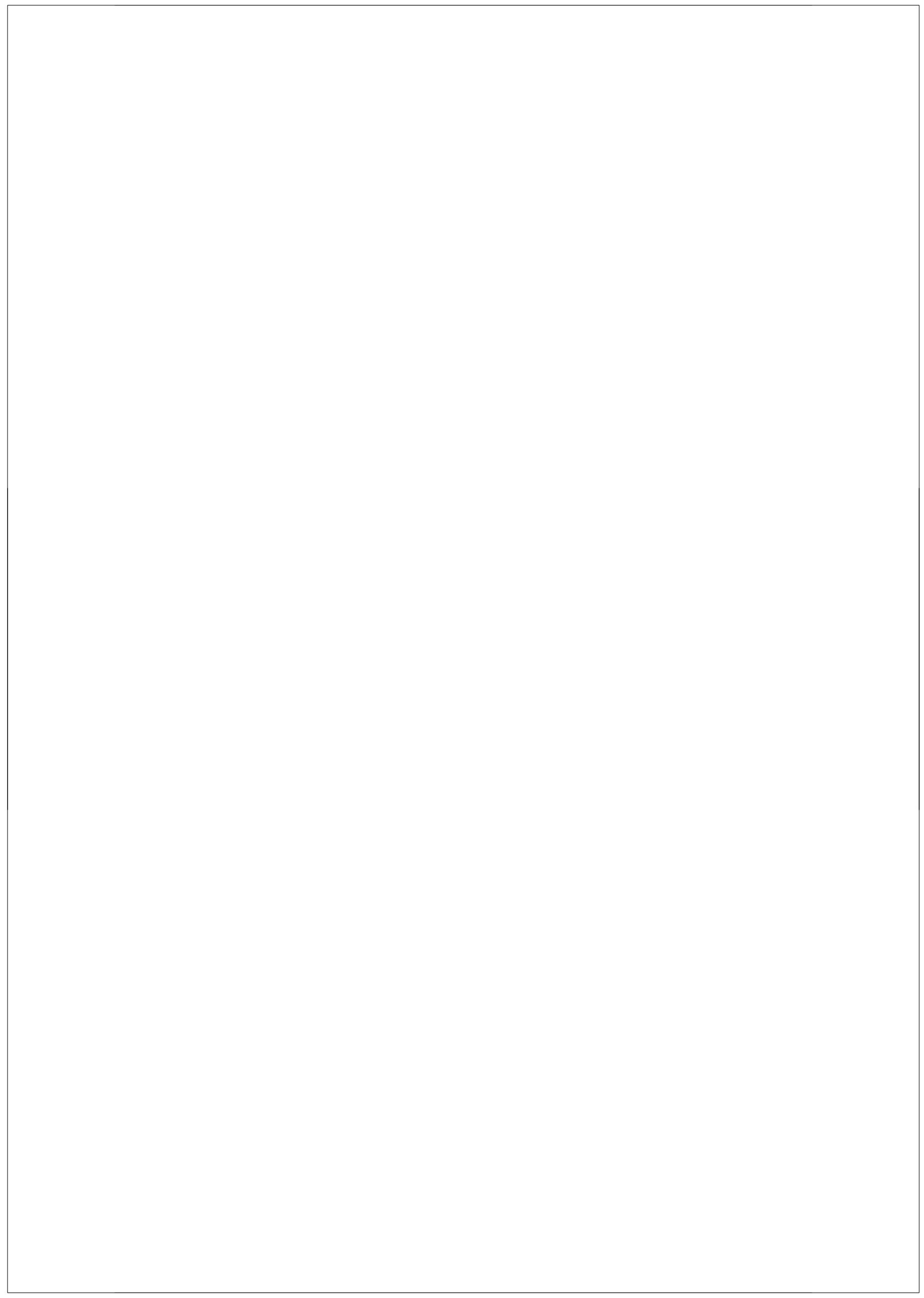

